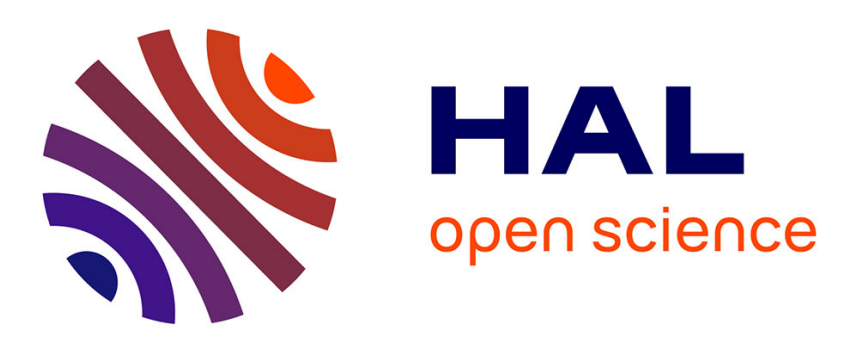

\title{
Recent developments on the stability of systems with aperiodic sampling: An overview
}

Laurentiu Hetel, Christophe Fiter, Hassan Omran, Alexandre Seuret, Emilia Fridman, Jean-Pierre Richard, Silviu Iulian Niculescu

\section{- To cite this version:}

Laurentiu Hetel, Christophe Fiter, Hassan Omran, Alexandre Seuret, Emilia Fridman, et al.. Recent developments on the stability of systems with aperiodic sampling: An overview. Automatica, 2017, 76, pp.309 - 335. 10.1016/j.automatica.2016.10.023 . hal-01363448

\section{HAL Id: hal-01363448 \\ https://hal.science/hal-01363448}

Submitted on 12 Sep 2016

HAL is a multi-disciplinary open access archive for the deposit and dissemination of scientific research documents, whether they are published or not. The documents may come from teaching and research institutions in France or abroad, or from public or private research centers.
L'archive ouverte pluridisciplinaire HAL, est destinée au dépôt et à la diffusion de documents scientifiques de niveau recherche, publiés ou non, émanant des établissements d'enseignement et de recherche français ou étrangers, des laboratoires publics ou privés. 


\title{
Recent developments on the stability of systems with aperiodic sampling: an overview *
}

\author{
Laurentiu Hetel ${ }^{\mathrm{a}, *}$ Christophe Fiter ${ }^{\mathrm{a}}$ Hassan Omran ${ }^{\mathrm{b}}$ Alexandre Seuret ${ }^{\mathrm{c}}$ \\ Emilia Fridman ${ }^{\mathrm{d}}$ Jean-Pierre Richard ${ }^{\mathrm{a}, \mathrm{e}}$ and Silviu Iulian Niculescu ${ }^{\mathrm{f}}$ \\ ${ }^{\mathrm{a}}$ CNRS CRISTAL UMR 9189, \\ École Centrale de Lille / Univ. Lille 1, 59651 Villeneuve d'Ascq, France \\ ${ }^{\mathrm{b}}$ ICube lab., UMR 7357 University of Strasbourg/CNRS, France \\ c CNRS, LAAS, 7 avenue du Colonel Roche, F-31400 Toulouse, France. \\ d School of Electrical Engineering, Tel-Aviv University, Tel-Aviv 69978, Israel. \\ ${ }^{\mathrm{e}}$ Non-A, INRIA Lille-Nord Europe, France \\ ${ }^{\mathrm{f}}$ Laboratoire des Signaux et Systèmes (L2S, UMR CNRS 8506), CNRS - Centrale SUPELEC - Univ. Paris Sud, 3 rue Joliot \\ Curie, 91192 Gif-sur-Yvette, France.

\begin{abstract}
This article presents basic concepts and recent research directions about the stability of sampled-data systems with aperiodic sampling. We focus mainly on the stability problem for systems with arbitrary time-varying sampling intervals which has been addressed in several areas of research in Control Theory. Systems with aperiodic sampling can be seen as time-delay systems, hybrid systems, Input/Output interconnections, discrete-time systems with time-varying parameters, etc. The goal of the article is to provide a structural overview of the progress made on the stability analysis problem. Without being exhaustive, which would be neither possible nor useful, we try to bring together results from diverse communities and present them in a unified manner. For each of the existing approaches, the basic concepts, fundamental results, converse stability theorems (when available), and relations with the other approaches are discussed in detail. Results concerning extensions of Lyapunov and frequency domain methods for systems with aperiodic sampling are recalled, as they allow to derive constructive stability conditions. Furthermore, numerical criteria are presented while indicating the sources of conservatism, the problems that remain open and the possible directions of improvement. At last, some emerging research directions, such as the design of stabilizing sampling sequences, are briefly discussed.
\end{abstract}

Key words: networked/embedded control systems, sampled-data control, aperiodic sampling, stability analysis, time-delay systems, hybrid systems, convex embeddings, Integral Quadratic Constraints.

\section{Introduction}

The last decade has witnessed an enormous interest in the study of networked and embedded control systems $[276,116,107,38]$. This interest is mainly due to the

\footnotetext{
* This work was supported by Region Nord-Pas de Calais through the ARCIR project ESTIREZ, by the ANR Project ROCC-SYS (agreement ANR-14-CE27-0008) and the CNRS GDRI DelSys.

* Corresponding author. Tel.: +33 (0)3 20676013

Email address: laurentiu.hetel@ec-lille.fr (Laurentiu Hetel).
}

ubiquitous presence of embedded controllers in relevant application domains and the growing demand in industry on systematic methods to model, analyse and design systems where sensor and control data are transmitted over a digital communication channel. The study of systems with aperiodic sampling emerged as a modelling abstraction which allows to understand the behaviour of Networked Control Systems (NCS) with sampling jitters, packet drop-outs or fluctuations due to the inter-action between control algorithms and real-time scheduling protocols $[276,7,11]$. With the emergence of event-based and self-triggered control techniques $[13,10,259,103]$, the study of aperiodic sampled-data 
systems constitutes nowadays a very popular research topic in control. In this survey, we focus on questions arising in the control of systems with arbitrary timevarying sampling intervals. Important practical questions such as the choice of the sampling frequency, the evaluation of necessary computational and energetic resources or the robust control synthesis are mainly related to stability issues. These issues often lead to the problem of estimating the Maximum Sampling Interval (MSI) for which the stability of a closed-loop sampleddata system is ensured.

The study of aperiodic sampled-data systems has been addressed in several areas of research in Control Theory. Systems with aperiodic sampling can be seen as particular time-delay systems. Sampled-and-hold in control and sensor signals can be modelled using hybrid systems with impulsive dynamics. Aperiodic sampled-data systems have also been studied in the discrete-time domain. In particular, Linear Time Invariant (LTI) sampled-data systems with aperiodic sampling have been analysed using discrete-time Linear Parameter Varying ( $L P V)$ models. The effect of sampling can be modelled using operators and the stability problem can be addressed in the framework of Input/Output interconnections as typically done in modern Robust Control. While significant advances on this subject have been presented in the literature, problems related to both the fundamentals of such systems and the derivation of constructive methods for stability analysis remain open, even for the case of linear system. The objective of the article is to present in a unified and structured manner a collection of significant results on this topic.

The core of the article is dedicated to the analysis of systems with arbitrary varying sampling intervals. We will only consider the deterministic aspects of the problem. The case when sampling intervals are random variables given by a probability distribution will not be discussed here. After presenting some generalities and motivations concerning sampled-data systems with aperiodic sampling (in Section 2), some basic qualitative results are recalled in Section 3. Section 4 presents the main stability analysis approaches. At last, in Section 5, we briefly discuss some emerging research problems, such as the design of stabilizing sampling sequences. We indicate the main challenges, the relations with the arbitrary sampling problem and some perspectives on which the current approaches and tools for aperiodic sampled-data systems may be useful in the future.

Notations: Throughout the paper, $\mathbb{R}_{+}$denotes the set $\{\lambda \in \mathbb{R}, \lambda \geq 0\},\|x\|$ represents any norm of the vector $x$ and $\|x\|_{p}, p \in \mathbb{N}$, the $p$ norm of a vector $x$. For a matrix $M, M^{T}$ denotes the transpose of $M$ and $M^{\star}$, its conjugate transpose. For square symmetric matrices $M, N$, $M \succeq N$ (resp. $M \succ N$ ) means that $M-N$ is a positive (resp. definite positive) matrix. $*$, in a symmetric matrix

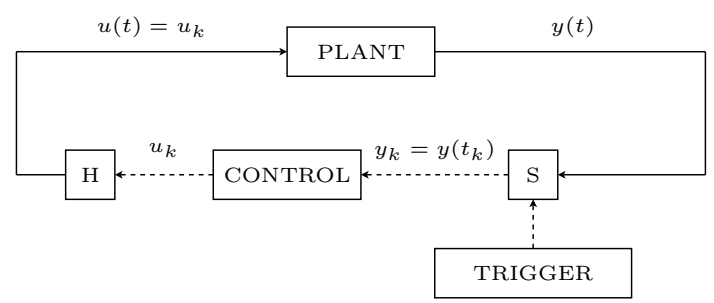

Fig. 1. Classical sampled-data system configuration

represents elements that may be induced by symmetry. $\|M\|_{p}, p \in \mathbb{N}$ denotes the induced $p$-norm of a matrix $M . \bar{\sigma}(M)$ denotes the maximum singular value of $M$. $\mathcal{C}^{0}(X, Y)$, for two metric spaces $X$ and $Y$, is the set of continuous functions from $X$ to $Y . \mathcal{L}_{p}^{n}(a, b), p \in \mathbb{N}$ denotes the space of functions $\phi:(a, b) \rightarrow \mathbb{R}^{n}$ with norm $\|\phi\|_{\mathcal{L}_{p}}=\left[\int_{a}^{b}\|\phi(s)\|^{p} d s\right]^{\frac{1}{p}}$, and $\mathcal{L}_{2 e}^{n}[0, \infty)$ is the space of functions $\phi:[0, \infty) \rightarrow \mathbb{R}^{n}$ which are square integrable on finite intervals.

\section{Generalities}

\subsection{System configuration}

In this paper we study the properties of sampled-data systems consisting of a plant, a digital controller, and appropriate interface elements. A general configuration of such a sampled-data system is illustrated by the block diagram of Figure 1. In this configuration, $y(t)$ is a continuous-time signal representing the plant output (the plant variables that can be measured). This signal is represented as a function of time $t, y: \mathbb{R}_{+} \rightarrow \mathbb{R}^{p}$.

The digital controller is usually implemented as an algorithm on an embedded computer. It operates with a sampled version of the plant output signal, $\left\{y_{k}\right\}_{k \in \mathbb{N}}$, obtained upon the request of a sampling trigger signal at discrete sampling instants $t_{k}$ and using an analog-todigital converter (the sampler block, $S$, in Figure 1 ). This trigger may represent a simple clock, as in the classical periodic sampling paradigm, or a more complex scheduling protocol which may take into account the sensor signal, a memory of its last sampled values, etc. The sampling instants are described by a monotone increasing sequence of positive real numbers $\sigma=\left\{t_{k}\right\}_{k \in \mathbb{N}}$ where

$$
t_{0}=0, t_{k+1}-t_{k}>0, \lim _{k \rightarrow \infty} t_{k}=\infty .
$$

The difference between two consecutive sampling times $h_{k}=t_{k+1}-t_{k}$ is called the $k^{t h}$ sampling interval. Assuming that the effect of quantizers may be neglected, the sampled version of the plant output is the sequence $\left\{y_{k}\right\}_{k \in \mathbb{N}}$ where $y_{k}=y\left(t_{k}\right)$.

In a sampled-data control loop, the digital controller produces a sequence of control values $\left\{u_{k}\right\}_{k \in \mathbb{N}}$ using the 
sampled version of the plant output signal $\left\{y_{k}\right\}_{k \in \mathbb{N}}$. This sequence is converted into a continuous-time signal $u(t)$, where $u: \mathbb{R}_{+} \rightarrow \mathbb{R}^{m}$ (corresponding to the plant input) via a digital-to-analog interface. We consider that the digital-to-analog interface is a zero-order hold (the hold block, $H$, in Figure 1). Furthermore, we assume that there is no delay between the sampling instant $t_{k}$ and the moment the control $u_{k}$ (obtained based on the $k^{t h}$ plant output sample, $y_{k}$ ) is effectively implemented at the plant input. Then the input signal $u(t)$ is a piecewise constant signal $u(t)=u\left(t_{k}\right)=u_{k}, \forall t \in\left[t_{k}, t_{k+1}\right)$.

In this survey, we will consider that the plant is modelled by a finite dimensional ordinary differential equation of the form

$$
\left\{\begin{array}{l}
\dot{x}=F(t, x, u) \\
y=H(t, x, u)
\end{array}\right.
$$

where $x \in \mathbb{R}^{n}$ represents the plant state-variable. Here $F: \mathbb{R}_{+} \times \mathbb{R}^{n} \times \mathbb{R}^{m} \rightarrow \mathbb{R}^{n}$ with $F(t, 0,0)=0, \forall t \geq 0$, and $H: \mathbb{R}_{+} \times \mathbb{R}^{n} \times \mathbb{R}^{m} \rightarrow \mathbb{R}^{p}$. It is assumed that for each constant control and each initial condition $\left(t_{0}, x_{0}\right) \in$ $\mathbb{R}_{+} \times \mathbb{R}^{n}$ the function $F$ describing the plant model (2) is such that a unique solution exists for an interval $\left[t_{0}, t_{0}+\epsilon\right)$ with $\epsilon$ large enough with respect to the maximum sampling interval. The discrete-time controller is considered to be described by an ordinary difference equation of the form

$$
\left\{\begin{aligned}
x_{k+1}^{c} & =F_{d}^{c}\left(k, x_{k}^{c}, y_{k}\right), \\
u_{k} & =H_{d}^{c}\left(k, x_{k}^{c}, y_{k}\right)
\end{aligned}\right.
$$

where $x_{k}^{c} \in \mathbb{R}^{n_{c}}$ is the controller state. Here, $F_{d}^{c}: \mathbb{N} \times$ $\mathbb{R}^{n_{c}} \times \mathbb{R}^{p} \rightarrow \mathbb{R}_{c}^{n}$ and $H_{d}^{c}: \mathbb{N} \times \mathbb{R}^{n_{c}} \times \mathbb{R}^{p} \rightarrow \mathbb{R}^{m}$. We will use the denomination sampled-data system for the interconnection between the continuous-time plant (2) with the discrete-time controller (3) via the relations

$$
y_{k}=y\left(t_{k}\right), u(t)=u_{k}, \forall t \in\left[t_{k}, t_{k+1}\right), \forall k \in \mathbb{N},
$$

under a sequence of sampling instants $\sigma=\left\{t_{k}\right\}_{k \in \mathbb{N}}$ satisfying (1).

The different concepts and results will be mostly illustrated on Linear Time Invariant (LTI) models

$$
\dot{x}=A x+B u
$$

under a static linear state feedback,

$$
u_{k}=K x_{k}, k \in \mathbb{N} \text {, }
$$

with $x_{k}=x\left(t_{k}\right)$. However, when possible, we will present extensions to more general nonlinear systems.

\subsection{Classical design methods}

There are various approaches for the design of a sampleddata controller (3) (see the classical textbooks [16,39] and the tutorial papers $[183,182,192,142])$.

Emulation. The simplest approach consists in designing first a continuous-time controller using classical methods $[136,123,140,229]$. Next, a discrete-time controller of the form (3) is obtained by integrating the controller solutions over the interval $\left[t_{k}, t_{k+1}\right)$. This approach is usually called emulation. Generally, it is difficult to compute in a formal manner the exact discrete-time model and approximations must be used $[183,142]$. In the LTI case (5) with state feedback (6), the emulation simply means that the gain $K$ is set such that the matrix $A+B K$ is Hurwitz and that the plant is driven by the control $u(t)=K x\left(t_{k}\right), \forall t \in\left[t_{k}, t_{k+1}\right), k \in \mathbb{N}$. While the intuition seems to indicate that for sufficiently small sampling intervals the obtained sampled-data control gives an approximation of the continuous-time control problem, no guarantee can be given when the sampling interval increases, even for constant sampling intervals. In order to compensate for the effect of controller discretisation, re-design methods may be used $[95,189]$.

Discrete-time design. In this framework, a discrete-time model of the plant (2) is derived by integration. The obtained model represents the evolution of the plant state $x\left(t_{k}\right)=x_{k}$ at sampling times ${ }^{1}$. Then, a discrete-time controller (3) is designed using the obtained discretetime model. In the simplest LTI case (5), (6), the evolution of the state between two consecutive sampling instants $t_{k}$ and $t_{k+1}$ is given by

$$
x(t)=\Lambda\left(t-t_{k}\right) x\left(t_{k}\right), \forall t \in\left[t_{k}, t_{k+1}\right], k \in \mathbb{N},
$$

with a matrix function $\Lambda$ defined on $\mathbb{R}$ as

$$
\Lambda(\theta)=A_{d}(\theta)+B_{d}(\theta) K=\mathrm{e}^{A \theta}+\int_{0}^{\theta} \mathrm{e}^{A s} d s B K .
$$

Evaluating the closed-loop system's evolution at $t=$ $t_{k+1}$ and using the notation $h_{k}=t_{k+1}-t_{k}$ leads to the linear difference equation

$$
x_{k+1}=\Lambda\left(h_{k}\right) x_{k}, \forall k \in \mathbb{N}
$$

representing the closed-loop system at sampling instants. When the sampling interval is constant,

\footnotetext{
1 Note that generally approximations of the system model must be used since the discretized plant model is difficult to compute formally $[181,261]$. Even for the case of LTI systems with constant sampling intervals, the numerical computation of the matrix exponential (or its integral) is subject to approximations [179].
} 
$h_{k}=T, \forall k \in \mathbb{N}$, a large variety of discrete-time control design methodologies is available in the literature (see $[16,39]$ and the references within). It is well known for this case that system (9) is asymptotically stable if and only if the matrix $\Lambda(T)$ is Schur. In other words, to design a stabilizing control law (6), the matrix $K$ must be set such as all the eigenvalues of $\Lambda(T)$ lay in the open unit disk.

For nonlinear systems with constant sampling intervals, an overview of control design methodologies and related issues can be found in $[183,182,192,142]$. Note that the discrete-time models such as (9) do not take into consideration the inter-sampling behaviour of the system. Relations between the performances of the discrete-time model and the performances of the sampled-data loop, can be deduced using the methodology proposed in [196].

Sampled-data design. Infinite dimensional discrete-time models which take into account the inter-sampling system behaviour using signal lifting [19,20,251,257,269] have been proposed in the literature for the case of linear systems. Specific design methodologies, which are able to take in consideration continuous-time system performances, inter-sample ripples and robustness specifications, can be found in the textbook [39] for the case of linear time invariant systems with periodic sampling.

\subsection{Complex phenomena in aperiodic sampling}

While in the last fifty years an intensive research has been dedicated to the analysis and design of sampleddata systems under periodic sampling, the study of systems with time-varying sampling intervals is quite underdeveloped compared to the periodic conterpart. The following examples illustrate the rich complexity of phenomena that may occur under aperiodic sampling.

Example 1 [274] Consider an LTI sampled-data system of the form (5),(6) where

$$
A=\left[\begin{array}{ll}
1 & 3 \\
2 & 1
\end{array}\right], \quad B=\left[\begin{array}{c}
1 \\
0.6
\end{array}\right], \quad K=-\left[\begin{array}{ll}
1 & 6
\end{array}\right]
$$

For this example, system's (9) transition matrix $\Lambda(T)$ is a Schur matrix for any constant sampling interval in $T \in \mathcal{T}=\left\{T_{1}, T_{2}\right\}$, with $T_{1}=0.18$, and $T_{2}=0.54$. Then, in the case of periodic sampling, the sampled-data system is stable for constant sampling intervals taking values in $\mathcal{T}$. An illustration of the system's evolution for constant sampling intervals $T_{1}, T_{2}$, is given in Figure 2. Clearly, when the sampling interval $h_{k}$ is arbitrarily varying in $\mathcal{T}$, the Schur property of $\Lambda(T), \forall T \in \mathcal{T}$, represents a necessary condition for stability of the sampleddata system (1),(5),(6). However, it is not a sufficient one. For example, the sampled-data system with a sequence of periodically time-varying sampling intervals
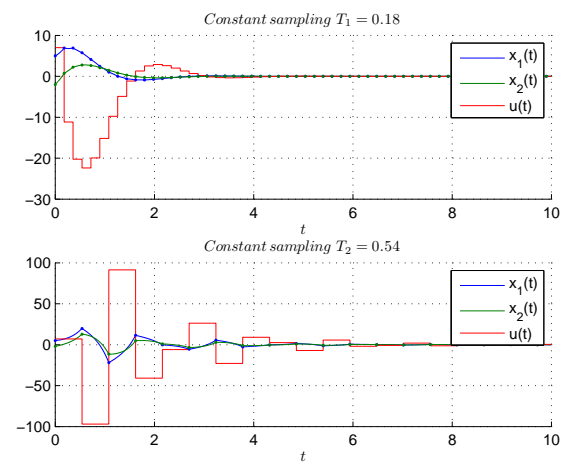

Fig. 2. Stability of the system in Example 1 with periodic sampling intervals.

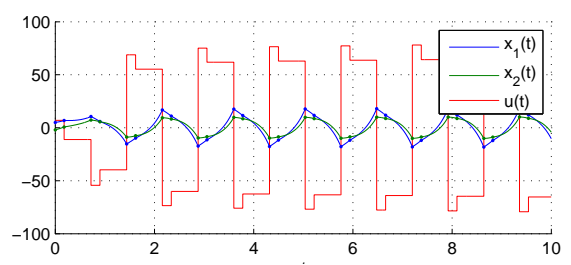

Fig. 3. Instability of the system in Example 1 with a periodic sampling sequence $T_{1} \rightarrow T_{2} \rightarrow T_{1} \cdots$.

$\left\{h_{k}\right\}_{k \in \mathbb{N}}=\left\{T_{1}, T_{2}, T_{1}, T_{2}, \ldots\right\}$ is unstable, as it can be seen in Figure 3. This is due to the fact that the Schur property of matrices is not preserved under matrix product (i.e. the product of two Schur matrices is not necessarily Schur). Indeed, the discrete-time system representation over the two sampling instants can be written as

$$
x_{k+2}=\Lambda\left(T_{2}\right) \Lambda\left(T_{1}\right) x_{k}, \forall k \in 2 \mathbb{N},
$$

and the transition matrix $\Lambda\left(T_{2}\right) \Lambda\left(T_{1}\right)$ over two sampling intervals $T_{1}$ and $T_{2}$, is not Schur. This example shows the importance of taking into consideration the evolution of the sampling interval $h_{k}$ when analysing the stability of sampled-data systems since variations of the sampling interval $h_{k}$ may induce instability.

Example 2 [96] Consider now an LTI system with

$$
A=\left[\begin{array}{cc}
0 & 1 \\
-2 & 0.1
\end{array}\right], B=\left[\begin{array}{l}
0 \\
1
\end{array}\right] K=\left[\begin{array}{ll}
1 & 0
\end{array}\right]
$$

Assume that the sampling interval $h_{k}$ is restricted to the set $\mathcal{T}=\left\{T_{1}, T_{2}\right\}$ with $T_{1}=2.126$ and $T_{2}=3.950$. The system is unstable for both constant sampling intervals $T_{1}$ and $T_{2}$ since for these values system's (9) transition matrix $\Lambda(T), T \in \mathcal{T}$ is not a Schur matrix. However, the product of transition matrices $\Lambda\left(T_{1}\right) \Lambda\left(T_{2}\right)$ has the Schur property. Therefore, the sampled-data system is stable under a periodic evolution of the sampling interval $\left\{h_{k}\right\}_{k \in \mathbb{N}}=\left\{T_{1}, T_{2}, T_{1}, T_{2}, \ldots\right\}$. An example of system evolution with this particular sampling sequence is 
provided in Figure 4. In this example the sampling $h_{k}$ can act on the sampled-data system as a second control parameter which ensures the system's stability while the possible constant sampling configurations are not able to guarantee this property.

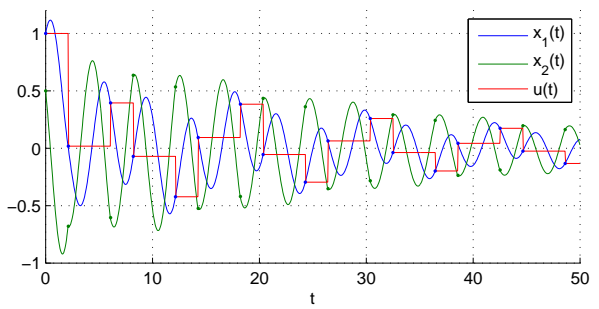

Fig. 4. Periodic sampling sequence with a stable behaviour.

\subsection{Problem set-ups}

The core of the survey is dedicated to the robust analysis of sampled-data systems with sampling sequences of the form (1) where the sampling interval $h_{k}=t_{k+1}-t_{k}$ takes arbitrary values in some set $\mathcal{T}=[\underline{h}, \bar{h}] \subset \mathbb{R}_{+}$. This first problem set-up may correspond, for example, to the sampling triggering mechanism from Figure 1 with a clock submitted to jitter [268], or with some scheduling protocol which is too complex to be modelled explicitly $[276,107]$. Basically, for the case of LTI models (5) with linear state feedback (6) under a sampling sequence (1) we will address the robust stability of the closed-loop system (12) given below

$$
\left\{\begin{aligned}
\dot{x}(t) & =A x(t)+B K x\left(t_{k}\right), \forall t \in\left[t_{k}, t_{k+1}\right), \forall k \in \mathbb{N} \\
t_{k+1} & =t_{k}+h_{k}, \forall k \in \mathbb{N} \\
t_{0} & =0, x\left(t_{0}\right)=x_{0} \in \mathbb{R}^{n}
\end{aligned}\right.
$$

as if $h_{k}$ is a time-varying "perturbation" taking values in a bounded set $\mathcal{T}$.

In Section 5 we briefly indicate some basic ideas concerning a recently emerging research topic where the sampling interval $h_{k}$ plays the role of a control parameter that may be changed according to the plant state or output. This problem set-up corresponds to the design of a scheduling mechanism. For the case of system (12), $h_{k}$ is considered as an additional input which, by an appropriate open/closed-loop choice, can ensure the system stability.

\section{Qualitative properties of sampled-data sys- tems}

In this section we recall some aspects concerning the qualitative behaviour of sampled-data systems with time-varying sampling intervals. First, we discuss the existence of a sufficiently small sampling interval that preserves asymptotic stability when discretizing a continuous-time stabilizing controller. Next, we present qualitative stability results which can be deduced for nonlinear sampled-data systems using linearization.

\subsection{Small sampling interval approximations}

The choice of sampling intervals is a critical issue in the emulation approach. Intuitively, choosing a sufficiently large sampling frequency should preserve the stability under a sampled-data implementation. This conjecture has been confirmed in $[254,105,106]$ for systems with periodic sampling. For the case of aperiodic sampling, various classes of systems have been treated in the literature $[118,34,210]$. The case of LTI systems (5) with linear state feedback (6) has been addressed long ago in [118].

Theorem 1 [118] Consider that system $\dot{x}=(A+B K) x$ is exponentially stable. Then there exists a scalar $\bar{h}>0$ such that the closed-loop system (12) is Exponentially Stable for any sequence $\sigma=\left\{t_{k}\right\}_{k \in \mathbb{N}}$ of the form (1) satisfying $h_{k}=t_{k+1}-t_{k}<\bar{h}, \forall k \in \mathbb{N}$.

The proof is based on the existence of a quadratic Lyapunov function for the continuous-time closed-loop system $\dot{x}=(A+B K) x$. An extension to a more general class of (input affine) nonlinear systems is given below.

Theorem 2 [118] Let $x=0$ be a globally exponentially stable equilibrium point of system $\dot{x}=f(x)+g(x) u$ with $u=K(x)$, where $f: \mathbb{R}^{n} \rightarrow \mathbb{R}^{n}, g: \mathbb{R}^{n} \rightarrow \mathbb{R}^{n \times m}$, $K: \mathbb{R}^{n} \rightarrow \mathbb{R}^{m}$, and let $V: \mathbb{R}^{n} \rightarrow \mathbb{R}_{+}$be a radially unbounded $\mathcal{C}^{1}$ function such that $V(0)=0, V(x)>0$ for all $x \neq 0$. Assume that the following conditions are satisfied:

- the functions $f($.$) and K($.$) are globally Lipschitz;$

- there exists $G>0$ such that $\|g(x)\| \leq G$ for all $x \in \mathbb{R}^{n}$;

- there exist $c_{1}, c_{2}>0$ such that for any $x \in \mathbb{R}^{n}$

$$
\frac{\partial V}{\partial x}(f(x)+g(x) K(x)) \leq-c_{1}\|x\|^{2},\left\|\frac{\partial V}{\partial x}\right\| \leq c_{2}\|x\| ;
$$

- given a closed set $B_{1}$ and a bounded open set $B_{2}$ with $B_{1} \subset B_{2} \subset \mathbb{R}^{n}$ there exists a scalar $s\left(B_{1}, B_{2}\right)>0$ such that, if the initial condition $\eta(0) \in B_{1}$, then the trajectory $\eta(t)$ of $\dot{\eta}=f(\eta)$ satisfies $\eta(t) \in B_{2}$, for all $t \leq s\left(B_{1}, B_{2}\right)$.

Then there exists a scalar $\bar{h}>0$ such that $x=0$ is a Globally Exponentially Stable Equilibrium point of the system

$$
\dot{x}(t)=f(x(t))+g(x(t)) K\left(x\left(t_{k}\right)\right),
$$

$t \in\left[t_{k}, t_{k+1}\right), k \in \mathbb{N}$, for any sequence of sampling instants $\sigma=\left\{t_{k}\right\}_{k \in \mathbb{N}}$ of the form (1) satisfying $h_{k}=$ $t_{k+1}-t_{k}<\bar{h}, \forall k \in \mathbb{N}$. 
In [118] it was further shown that if the continuous-time control system $\dot{x}=f(x)+g(x) K(x)$ is asymptotically stable (instead of exponentially stable), then only practical stability is guaranteed for the sampled-data system (13). A more general case, dealing with the emulation of dynamical controllers based on Euler discretization was provided in [34,118]. An alternative to Theorem 2 can be found in [210] and concerns the same issue but the drift $f(x)$ is not required to satisfy any Lipschitz property. However, the continuous-time control loop should ensure the exponential decay of a quadratic Lyapunov function along its solutions. Furthermore, the result only states the practical stability of the sampled-data control loop. Another extension to a more general class of Networked Control Systems can be found in [194]. There it is shown that if a continuous-time controller is designed such that it yields input-to-state stability with respect to external disturbances, then the same controller will achieve a semi-global practical input-to-state stability property when implemented in a sampled-data control loop via an exact emulation. Qualitative results for the existence of both a sufficiently small sampling interval and a stabilizing sampled-data controller can be found in [133].

\subsection{Linear approximations}

The study of sampled-data systems with linear models and controllers is often easier to address than the nonlinear case. For some classes of nonlinear sampled-data systems, local stability can be deduced from the properties of a linearized model around the equilibrium $[115,119]$.

Consider the following nonlinear system

$$
\left\{\begin{array}{l}
\dot{x}=F(x, u) \\
y=H(x)
\end{array}\right.
$$

the discrete-time controller

$$
\left\{\begin{array}{c}
x_{k+1}^{c}=F_{d}^{c}\left(x_{k}^{c}, y_{k}\right) \\
u_{k}=H_{d}^{c}\left(x_{k}^{c}, y_{k}\right)
\end{array}\right.
$$

and the interconnection $y_{k}=y\left(t_{k}\right), u(t)=u_{k}, \forall t \in$ $\left[t_{k}, t_{k+1}\right), \forall k \in \mathbb{N}$, for sampling sequences $\sigma=\left\{t_{k}\right\}_{k \in \mathbb{N}}$ as defined in (1). The closed-loop system can be represented by the set of equations

$$
\left\{\begin{array}{l}
\dot{x}(t)=f\left(x(t), x_{k}, x_{k}^{c}\right), t \in\left[t_{k}, t_{k+1}\right) \\
x_{k+1}^{c}=g\left(x_{k}, x_{k}^{c}\right), k \in \mathbb{N},
\end{array}\right.
$$

where $f\left(x, x_{k}, x^{c}\right)=F\left(x, H_{d}^{c}\left(x_{k}^{c}, H\left(x_{k}\right)\right)\right), g\left(x_{k}, x_{k}^{c}\right)=$ $F_{d}^{c}\left(x_{k}^{c}, H\left(x_{k}\right)\right)$ and $x_{k}=x\left(t_{k}\right)$. For $f(x, v, w)$ and $g(v, w)$ let $A=\left.\frac{\partial f}{\partial x}\right|_{0}, A_{0}=\left.\frac{\partial f}{\partial v}\right|_{0}, B=\left.\frac{\partial f}{\partial w}\right|_{0}$,
$C=\left.\frac{\partial g}{\partial w}\right|_{0}, D=\left.\frac{\partial g}{\partial v}\right|_{0}$. To system (16) the following linear model is associated

$$
\left\{\begin{array}{l}
\dot{x}(t)=A x(t)+A_{0} x_{k}+B x_{k}^{c}, t \in\left[t_{k}, t_{k+1}\right), \\
x_{k+1}^{c}=C x_{k}^{c}+D x_{k}, k \in \mathbb{N} .
\end{array}\right.
$$

Integrating the system over a sampling interval and letting $z_{k}^{T}=\left[x_{k}^{T} x_{k}^{c T}\right]$ leads to the following linear timevarying discrete-time system

$$
z_{k+1}=\Omega\left(h_{k}\right) z_{k}, \forall k \in \mathbb{N}
$$

with

$$
\Omega\left(h_{k}\right)=\left[\begin{array}{cc}
e^{A h_{k}}+\int_{0}^{h_{k}} e^{A s} d s A_{0} & \int_{0}^{h_{k}} e^{A s} d s B \\
D & C
\end{array}\right] .
$$

The following theorem establishes conditions for the stability of the nonlinear system (16) under arbitrary variations of the sampling interval.

Theorem 3 [119] Assume that, for every possible sequence $\sigma=\left\{t_{k}\right\}_{k \in \mathbb{N}}$ defined in (1), one has $h_{k}=t_{k+1}-$ $t_{k} \leq \bar{h}$, and for any $k \in \mathbb{N},\left\|\Omega\left(h_{k}\right)\right\|_{2}<q<1$, where $\bar{h}$ and $q$ are constant scalars. Then the equilibrium point $\left[x^{T} x^{c T}\right]=0$ of system (16) is Exponentially Stable.

The nature of the result is in the spirit of the Lyapunov's first method [136], as it permits to guarantee the stability of the equilibrium of the nonlinear system, by studying the stability of its linearization at the origin. In the same way, it remains qualitative and it does not provide any estimate of the domain of attraction. However, the result does not require the sampling intervals to be small.

The following theorem uses the linear model (18) in order to provide conditions for the stability of the nonlinear sampled-data system (16) with a fixed sequence of sampling instants.

Theorem 4 [119] Let $\sigma=\left\{t_{k}\right\}_{k \in \mathbb{N}}$ be a sequence defined in (1) with $\sup _{k \in \mathbb{N}}\left\{t_{k+1}-t_{k}\right\}=\bar{h}<\infty$, where $\bar{h}$ is a given constant. Assume that either

(i) $\lim \sup _{k \rightarrow \infty}\left\|\Omega\left(h_{k}\right)\right\|_{2}<1$, or

(ii) $\limsup _{k \rightarrow \infty}\left\{\max \left|\operatorname{eig}\left(\Omega_{k}\right)\right|\right\}<1$ and every subsequence of $\left\{\Omega\left(h_{k}\right)\right\}_{k \in \mathbb{N}}$ converges to a Schur matrix and the solutions $P_{k}$ of $\Omega^{T}\left(h_{k}\right) P_{k} \Omega\left(h_{k}\right)-P_{k}=-I$ satisfy $\lim \sup _{k \rightarrow \infty}\left\|P_{k+1}-P_{k}\right\|_{2}<1$, or

(iii) $\Omega\left(h_{k}\right)$ converges to a Schur matrix. 
Then the equilibrium point $\left[x^{T} x^{c T}\right]=0$ of system (16) with the sampling sequence $\sigma$ is Exponentially Stable.

Note that, in the conditions of Theorem 4, the matrix $\Omega\left(h_{k}\right)$ is not required to be Schur for all the values of $k \in \mathbb{N}$. One may find particular sequences of sampling instants $\sigma=\left\{t_{k}\right\}_{k \in \mathbb{N}}$ satisfying the conditions i) or iii) in Theorem 4 for which the eigenvalues of $\Omega\left(h_{k}\right)$ are outside the unit disk for some values of $k$. The result is interesting when the sampling interval $h_{k}$ can be considered as a control parameter, for scheduling the sampling instants in an appropriate manner. The theorem may be used to determine scheduling mechanisms with sampling intervals larger than in a periodic sampling configuration.

\section{Stability analysis under arbitrary time- varying sampling}

The previous results are qualitative and prove some nice properties of sampled-data systems. However, they do not provide any method for estimating the set of sampling intervals for which the stability properties are still guaranteed. In the following, we review some results which provide such an estimation for sampled-data systems with sampling intervals that are arbitrary varying. More formally, over the section, we present results that address the following problem:

- Problem A (Arbitrary sampling problem): Consider the sampled-data system $(1),(2),(3),(4)$ and a bounded subset $\mathcal{T} \subset \mathbb{R}_{+}$. Determine if the sampleddata system is stable (in some sense) for any arbitrary time-varying sampling interval $h_{k}=t_{k+1}-t_{k}$ with values in $\mathcal{T}$.

Often the set $\mathcal{T}$ is considered of the form $\mathcal{T}=(0, \bar{h}]$ where $\bar{h}$ is some positive scalar. The largest value of $\bar{h}$ for which the stability of the closed loop system is ensured is called Maximum Sampling Interval (MSI).

Several perspectives for addressing Problem A exist. First, we present results that are based on a time-delay modelling of the sampled-data system $(1),(2),(3),(4)$. Next, we show how the problem can be addressed from the point of view of hybrid systems. We continue with approaches that use the explicit system integration inbetween successive sampling instants, such as the ones classically used in the discrete-time framework. Last, results addressing Problem A from the robust control theory point of view are presented.

\subsection{Time-delay approach}

To the best of our knowledge, this technique was initiated in $[172,15]$, and further developed in $[66,254,158]$
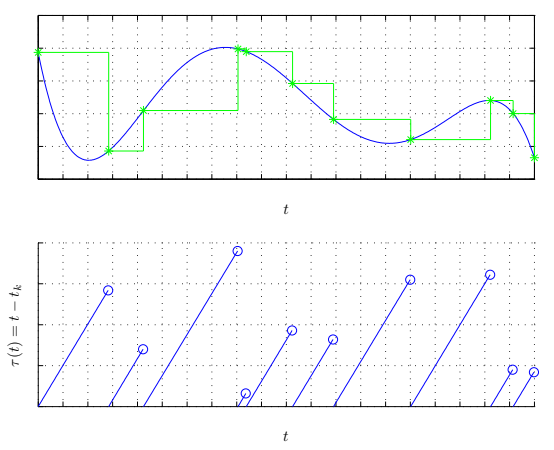

Fig. 5. Sampling seen as a piecewise-continuous time-delay.

and in several other works. For the case of an LTI system with sampled-data state feedback (12), we may re-write

$$
\begin{aligned}
& u(t)=K x\left(t_{k}\right)=K x(t-\tau(t)), \\
& \tau(t)=t-t_{k}, \quad \forall t \in\left[t_{k}, t_{k+1}\right),
\end{aligned}
$$

where the delay $\tau$ is piecewise-linear, satisfying $\dot{\tau}(t)=$ 1 for $t \neq t_{k}$, and $\tau\left(t_{k}\right)=0$. This delay indicates the time that has passed since the last sampling instant. An illustration of a typical delay evolution is given in Figure 5. The LTI system with sampled-data (12) is then re-modeled as an LTI system with a time-varying delay

$$
\dot{x}(t)=A x(t)+B K x(t-\tau(t)), \forall t \geq 0 .
$$

This permits to adapt the tools for the analysis of systems with fast varying delays $[76,97,225,198]$. This model is equivalent to the original sampled-data system when considering that the sampling induced delay has a known derivative $\dot{\tau}(t)=1$, for all $t \in\left[t_{k}, t_{k+1}\right), k \in \mathbb{N}$.

\subsubsection{Theoretical foundation}

For system (21) it is natural to consider, as a state variable, the functional $x_{t}(\theta)=x(t+\theta), \forall \theta \in[-\bar{h}, 0]$, and, as state space, the set $\mathcal{C}^{0}\left([-\bar{h}, 0], \mathbb{R}^{n}\right)$ of continuous functions mapping the interval $[-\bar{h}, 0]$ into $\mathbb{R}^{n}$ $[68,197,199]$. The most popular generalization of the direct Lyapunov method for time-delay system has been proposed by Krasovskii [139]. It uses the existence of functionals $V\left(t, x_{t}\right)$ depending on the state vector $x_{t}$. In the sampled-data case $[72,67,150]$ functionals $V\left(t, x_{t}, \dot{x}_{t}\right)$ depending both on $x_{t}$ and $\dot{x}_{t}$ (see [137], p.337) are useful.

Denote by $W[-\bar{h}, 0]$ the Banach space of absolutely continuous functions $\phi:[-\bar{h}, 0] \rightarrow \mathbb{R}^{n}$ with $\dot{\phi} \in \mathcal{L}_{2}^{n}(-\bar{h}, 0)$ (the space of square integrable functions) with the norm

$$
\|\phi\|_{W}=\max _{s \in[-\bar{h}, 0]}\|\phi(s)\|+\left[\int_{-\bar{h}}^{0}\|\dot{\phi}(s)\|^{2} d s\right]^{\frac{1}{2}} .
$$




\section{Theorem 5 (Lyapunov-Krasovskii Theorem)}

[137] Consider $f: \mathbb{R}_{+} \times \mathcal{C}^{0}[-\bar{h}, 0] \rightarrow \mathbb{R}^{n}$ continuous in both arguments and locally Lipschitz in the second argument. Assume that $f(t, 0)=0$ for all $t \in \mathbb{R}_{+}$and that $f$ maps $\mathbb{R} \times$ (bounded sets in $\mathcal{C}^{0}[-\bar{h}, 0]$ ) into bounded sets of $\mathbb{R}^{n}$. Suppose that $\alpha, v, w: \mathbb{R}_{+} \rightarrow \mathbb{R}_{+}$are continuous nondecreasing functions, $\alpha(s), \beta(s)$ and $\gamma(s)$ are positive for $s>0, \lim _{s \rightarrow \infty} \alpha(s)=\infty$ and $\alpha(0)=\beta(0)=0$. The trivial solution of

$$
\dot{x}(t)=f\left(t, x_{t}\right)
$$

is Globally Uniformly Asymptotically Stable if there exists a continuous functional $V: \mathbb{R} \times W[-\bar{h}, 0] \times$ $\mathcal{L}_{2}^{n}(-\bar{h}, 0) \rightarrow \mathbb{R}_{+}$, which is positive-definite, i.e.

$$
\alpha(\|\phi(0)\|) \leq V(t, \phi, \dot{\phi}) \leq \beta\left(\|\phi\|_{W}\right),
$$

for all $\phi \in W[-\bar{h}, 0], t \in \mathbb{R}_{+}$, and such that its derivative along the system's solutions is non-positive

$$
\dot{V}\left(t, x_{t}, \dot{x}_{t}\right) \leq-\gamma\left(\left\|x_{t}(0)\right\|\right)
$$

The functional $V$ satisfying the conditions of Theorem 5 is called a Lyapunov-Krasovskii Functional (LKF). In the general case of sampled-data nonlinear systems, the underlying delay system $\dot{x}=f\left(t, x_{t}\right)$ used in Theorem 5 from [137] is described by a function $f$ which is piecewise continuous with respect to $t$. However, the proof of the result in [137] can be adapted to cover this case. For the case of sampled-data systems, in [72] the LyapunovKrasovskii Theorem was extended to linear systems with a discontinuous sawtooth delay by use of the Barbălat lemma [21]. Another extension to linear sampled-data systems has been provided in [67], where the LKF is allowed to have discontinuities at sampling times.

\subsubsection{Tools and basic steps}

The derivation of stability conditions using LKFs usually involves quite elaborate developments. To give an idea of the procedure involved in this approach and to provide a glimpse of its technical flavour, we present first the derivation of LMI stability conditions for the case of LTI systems (12) with the associated time delay model (21). Based on elementary considerations, we expose the main difficulties and the most relevant tools. The basic steps for deriving constructive stability conditions are illustrated as follows.

Step 1. Propose a candidate Lyapunov-Krasovskii functional $V$. The LKF that is necessary and sufficient for the stability of LTI systems with delay has a rather complex form, even for the case of constant delays [138,71]. In order to provide constructive stability conditions, simplified forms are used, such as

$$
V\left(x_{t}, \dot{x}_{t}\right)=x^{T}(t) P x(t)+\bar{h} \int_{-\bar{h}}^{0} \int_{t+\theta}^{t} \dot{x}^{T}(s) R \dot{x}(s) \mathrm{d} s \mathrm{~d} \theta
$$

with $P, R \succ 0$, which was considered in [75].

Step 2. Compute the derivative of $V$. For the functional (23) this leads to

$$
\begin{aligned}
\dot{V}\left(x_{t}, \dot{x}_{t}\right) & =2 \dot{x}^{T}(t) P x(t)+\bar{h}^{2} \dot{x}^{T}(t) R \dot{x}(t) \\
& -\bar{h} \int_{t-\bar{h}}^{t} \dot{x}^{T}(s) R \dot{x}(s) \mathrm{d} s .
\end{aligned}
$$

The difficulty now comes from the last integral term, $J\left(\dot{x}_{t}, \bar{h}\right)=-\int_{t-\bar{h}}^{t} \dot{x}^{T}(s) R \dot{x}(s) \mathrm{d} s$, which is an impediment to the analysis of the sign of (24). Such terms are common in the derivative of LKFs and they need to be taken into account in an appropriated manner.

Step 3. Over-approximate the integral terms. This pro-

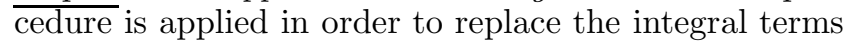
by more simple expressions. Unavoidably, using such over-approximations introduces some conservatism in the analysis. A relevant tool is Jensen's inequality, which is recalled here.

Lemma 6 (Jensen's inequality) [96] Given $R \succ 0$, $\theta \geq 0$, and a differentiable function $x:[t-\theta, t] \rightarrow \mathbb{R}^{n}$, the following inequality holds:

$$
\begin{gathered}
J\left(\dot{x}_{t}, \theta\right)=-\int_{t-\theta}^{t} \dot{x}(s) R \dot{x}(s) \mathrm{d} s \leq \\
-\frac{1}{\theta}(x(t)-x(t-\theta))^{T} R(x(t)-x(t-\theta)) .
\end{gathered}
$$

For the case presented in (24), splitting the integral in two terms and applying Jensen's inequality leads to

$$
\begin{aligned}
\frac{\mathrm{d}}{\mathrm{dt}} V\left(x_{t}, \dot{x}_{t}\right) & \leq 2 \dot{x}^{T}(t) P x(t)+\bar{h}^{2} \dot{x}^{T}(t) R \dot{x}(t) \\
& -\zeta(t)^{T} \bar{R}(\tau(t)) \zeta(t) \\
\text { where } \zeta(t)= & {\left[\begin{array}{c}
x(t)-x(t-\tau(t)) \\
x(t-\tau(t))-x(t-\bar{h})
\end{array}\right], \text { and } } \\
& \bar{R}(\tau(t))=\left[\begin{array}{cc}
\frac{\bar{h}}{\tau(t)} R & 0 \\
0 & \frac{\bar{h}}{h-\tau(t)} R
\end{array}\right]
\end{aligned}
$$

is a matrix depending on the delay value. At this step, sufficient conditions of stability are provided by the negativity of the right-hand side of the inequality (26). However, the obtained conditions need to be checked for all 
the values of $\tau(t) \in(0, \bar{h}]$, due to the dependence on $\tau(t)$ of the matrix $\bar{R}($.$) .$

Step 4. Over-approximate the delay dependent terms. The last step consists in over-approximating the elements that depend on $\tau(t)$ by simpler expressions which are constant or depend only on the upper bound $\bar{h}$. For example, in [72], stability conditions are obtained by noting that $\frac{\bar{h}}{\tau(t)} \geq 1$ and $\frac{\bar{h}}{h-\tau(t)} \geq 0$, which implies that

$$
\bar{R}(\tau(t)) \succeq\left[\begin{array}{ll}
R & 0 \\
0 & 0
\end{array}\right]
$$

holds for all delay $\tau$ in the interval $[0, \bar{h}]$. Of course, this over-approximation still introduces some conservatism in the analysis. However, for the case of LTI systems (12), over-approximating the terms in (26) leads to

$$
\frac{\mathrm{d}}{\mathrm{dt}} V\left(x_{t}, \dot{x}_{t}\right) \leq\left[\begin{array}{c}
x(t) \\
x(t-\tau(t))
\end{array}\right]^{T} \Psi(P, R)\left[\begin{array}{c}
x(t) \\
x(t-\tau(t))
\end{array}\right]
$$

with

$$
\begin{gathered}
\Psi(P, R)=\left[\begin{array}{cc}
P A+A^{T} P & P(B K) \\
(B K)^{T} P & 0
\end{array}\right] \\
+\bar{h}^{2}\left[\begin{array}{c}
A^{T} \\
(B K)^{T}
\end{array}\right] R[A B K]-\left[\begin{array}{c}
I \\
-I
\end{array}\right] R[I-I],
\end{gathered}
$$

from which LMI stability conditions can be derived $[75,72]$ simply by checking the negative definiteness of $\Psi(P, R)$.

Theorem 7 (adapted from [75,72]) Assume that there exists $P \succ 0$ and $R \succ 0$, such that the following linear matrix inequality $\Psi(P, R) \prec 0$ holds with $\Psi(P, R)$ as defined in (28). Then, the sampled-data system (12) is Asymptotically Stable for all sampling sequences $\sigma=$ $\left\{t_{k}\right\}_{k \in \mathbb{N}}$ with $h_{k}=t_{k+1}-t_{k} \leq \bar{h}$.

Theorem 7 is a simplified reformulation of the result in [72], where a more general case of polytopic systems is considered using a descriptor model $[75,74]$.

\subsubsection{Directions of improvement}

The main sources of conservatism in this approach are due to the choice of the LKF (Step 1) and the overapproximation of its derivative (Steps 3 and 4 ).

Step 1. The conservatism related to Step 1 can be reduced by adding new integral components to the LKF. For example the triple integral term
$V\left(\dot{x}_{t}\right)=\frac{\bar{h}^{2}}{2} \int_{-\bar{h}}^{0} \int_{\theta}^{0} \int_{t+\lambda}^{t} \dot{x}^{T}(s) R \dot{x}(s) \mathrm{d} s \mathrm{~d} \lambda \mathrm{d} \theta$ introduced by [246] for systems with fast varying delay might also be useful in the sampled-data case. For other forms of LKF see also $[68,211,240,9,236]$. We point in particular to the results in $[67,154,187,232]$, which have been specifically developed for the analysis of sampled-data systems. There, the proposed LKFs allow for taking into account the particular sawtooth evolution of the sampling induced delay $\left(\dot{\tau}(t)=1, \forall t \in\left[t_{k}, t_{k+1}\right)\right)$ while in the classical fast varying delay approach the delay derivative is assumed to be unknown and arbitrary varying. For example, it has been shown in [67] that the standard time-independent term $\int_{-\bar{h}}^{0} \int_{t+\theta}^{t} \dot{x}^{T}(s) R \dot{x}(s) d s d \theta$ used in [72] can be advantageously replaced by the term $\left(t_{k+1}-t\right) \int_{t_{k}}^{t} \dot{x}^{T}(s) R \dot{x}(s) d s$, which provides derivativedependent stability conditions. It leads to an LKF of the form [67]:

$$
\begin{gathered}
V\left(t, x(t), \dot{x}_{t}\right)= \\
x^{T}(t) P x(t)+\left(h_{k}-\tau(t)\right) \int_{t-\tau(t)}^{t} \dot{x}^{T}(s) R \dot{x}(s) d s
\end{gathered}
$$

which improves (23), as the information $\dot{\tau}=1$ can be explicitly taken into account when evaluating its derivative in Step 2. See also [230] for an alternative LMI formulation.

Step 3. Concerning the conservatism related to the overapproximation of integral terms (Step 3), the accuracy of Jensen's inequality has been addressed in [94,26,233]. Alternatively to the use of Jensen's inequality, the integral terms may also be over-approximated using extensions of Wirtinger's inequality [154,233,234,98]. Further refinements were developed in [145], where the authors considered a discretized version of this inequality, in [273], where additional free-weighted matrices are introduced or in [214] using auxiliary functions. Other improvements have been presented in $[234,235]$ based on Bessel's inequality and Legendre polynomials. Finally, following the description given in Step 1, the application of the Wirtinger-based inequality to the case of triple integral-type of LKFs was considered in [212].

Step 4. The reduction of the conservatism induced by the over-approximations of delay dependent terms (Step 4) has been considered by several authors over the last few years $[100,213,239]$. To the best of our knowledge, for the moment, the most accurate over-approximation of delay dependent terms is provided in [213]. This result encompasses several of the existing approximation techniques proposed in the literature $[72,100]$ and allows for an LMI formulation.

\subsubsection{A more recent result}

Clearly, considering more complex LKFs and more advanced over-approximation techniques increases the 
complexity of the proposed LMI stability criteria (in terms of readability). In the sequel, we present the simple stability conditions from [67], which take into account some of the presented conservatism reduction techniques and provide a fair compromise between accuracy and complexity.

Theorem 8 [67] Let there exist $P \succ 0, R \succ 0, P_{2}$ and $P_{3}$ such that the LMI

$$
\begin{gathered}
{\left[\begin{array}{ccc}
\Phi_{s} & P-P_{2}^{T}+(A+B K)^{T} P_{3} \\
* & -P_{3}-P_{3}^{T}+\bar{h} R
\end{array}\right] \prec 0,} \\
{\left[\begin{array}{ccc}
\Phi_{s} & P-P_{2}^{T}+(A+B K)^{T} P_{3} & -\bar{h} P_{2}^{T} A \\
* & -P_{3}-P_{3}^{T} & -\bar{h} P_{3}^{T} A \\
* & * & -\bar{h} R
\end{array}\right] \prec 0,}
\end{gathered}
$$

with $\Phi_{s}=P_{2}^{T}(A+B K)+(A+B K)^{T} P_{2}$, are feasible. Then system (12) is Exponentially Stable for all sampling sequences $\sigma=\left\{t_{k}\right\}_{k \in \mathbb{N}}$ with $h_{k}=t_{k+1}-t_{k} \leq \bar{h}$.

The result takes into account information about the sawtooth shape of the delay, which is the specificity of the time-delay model (21) when representing exactly the sampled-data system (12). It can ensure the stability for time-varying delays $\tau(t)$ which are longer than any constant delay that preserves stability, provided that $\dot{\tau}(t)=1$.

The research on LKFs for sampled-data systems is still a wide-open domain. Currently, an important effort is dedicated to finding better LKFs and better overapproximations of the derivatives. Note that providing improvements (in terms of conservatism reduction) at one step usually requires changes at all the others steps. For this reason, the derivation of constructive stability conditions may be quite an elaborate analytical process and it is not always very intuitive. However, a notable advantage of this methodology is the fact that for linear systems the approach can be easily extended to control design $[72,248,150]$ and to the case of systems with parameter uncertainties $[67,232,208,88,216]$, delays $[249,164,87,165,231,48]$ and schedulling protocols $[152,153,151]$. See also $[113,112]$ for the use of LKFs in the case of systems with switching control and $[70,69]$ for the control of semilinear 1-D heat equations.

\subsubsection{An extension to nonlinear systems}

Concerning nonlinear systems, [163] has extended the ideas in [72] for the case of control affine non-autonomous systems. Consider the nonlinear system:

$$
\dot{x}(t)=f(t, x(t))+g(t, x(t)) u(t),
$$

with the state $x(t) \in \mathbb{R}^{n}$ and the input $u(t) \in \mathbb{R}^{m}$, and with functions $f, g$ that are locally Lipschitz with respect to $x$ and piecewise continuous in $t$. Assume that a $\mathcal{C}^{1}$ controller $u(t)=K(t, x)$ is designed in order to make the system (32) Globally Uniformly Asymptotically Stable. Moreover, assume that there exist a $\mathcal{C}^{1}$ positive definite and radially unbounded function $V$, and a continuous positive definite function $W$ such that:

$$
-\left[\frac{\partial V}{\partial t}(t, x)+\frac{\partial V}{\partial x}(f(t, x)+g(t, x) K(t, x))\right] \geq W(x),
$$

for all $t \geq t_{0}$ and $x \in \mathbb{R}^{n}$. Also, consider $K(t, 0)=0$ for all $t \in \mathbb{R}$. Hence, $V$ is a strict Lyapunov function for $\dot{x}=f(t, x)+g(t, x) K(t, x)$, and one can fix class $\mathcal{K}_{\infty}$ functions $\alpha_{1}$ and $\alpha_{2}$ such that $\alpha_{1}\left(\|x\|_{2}\right) \leq V(t, x) \leq$ $\alpha_{2}\left(\|x\|_{2}\right)$, for all $t \geq t_{0}$ and $x \in \mathbb{R}^{n}$. Define the function

$$
\rho(t, x)=\frac{\partial K}{\partial t}(t, x)+\frac{\partial K}{\partial x}(f(t, x)+g(t, x) K(t, x)) .
$$

Theorem 9 (adapted from [163]) Suppose that there exist constants $c_{1}, c_{2}, c_{3}$ and $c_{4}$ such that:

$$
\begin{gathered}
\left\|\frac{\partial K}{\partial x}(t, x) g(t, x)\right\|_{2}^{2} \leq c_{1},\left\|\frac{\partial V}{\partial x}(t, x) g(t, x)\right\|_{2}^{2} \leq c_{2}, \\
\|\rho(t, x)\|_{2}^{2} \leq c_{3} W(x), \\
\left\|\frac{\partial V}{\partial x}(t, x) g(t, x) K(t, x)\right\|_{2} \leq c_{4}(V(t, x)+1),
\end{gathered}
$$

hold for all $t \geq t_{0}$ and $x \in \mathbb{R}^{n}$. Consider the system (32) in closed-loop with: $u(t)=K\left(t_{k}, x\left(t_{k}\right)\right), t \in\left[t_{k}, t_{k+1}\right)$, $\sigma=\left\{t_{k}\right\}_{k \in \mathbb{N}}$ as defined in (1) and $h_{k}=t_{k+1}-t_{k} \in[\underline{h}, \bar{h}]$, $\forall k \in \mathbb{N}$. Then, the closed-loop system is Globally Uniformly Asymptotically Stable if $\bar{h} \leq\left(4 c_{1}+8 c_{2} c_{3}\right)^{-1 / 2}$.

The stability is proven by means of a Lyapunov functional of the form

$$
U\left(t, x_{t}\right)=V(t, x(t))+\frac{\epsilon}{\bar{h}} \int_{-\bar{h}}^{0} \int_{t+\theta}^{t}\left\|\Psi\left(s, x_{s}\right)\right\|_{2}^{2} d s d \theta
$$

where $\Psi\left(t, x_{t}\right)=\frac{\partial K}{\partial t}\left(t, x_{s}(0)\right)+\frac{\partial K}{\partial x}\left(t, x_{t}(0)\right) \dot{x}_{t}(0)$ and $\epsilon>0$. This functional is reminiscent of the form (29) used in [72] to study LTI systems. However, differently from the LTI case, it is far more complex to determine how conservative the result is.

\subsubsection{Further reading}

Aside from the Lyapunov-Krasovskii method, the stability of sampled-data systems can also be analysed using the method proposed by Razumikhin [224]. Connections between Razumikhin's method and the ISS nonlinear small gain theorem [243] have been established in [253]. This relation has been used in [254] in order to show the preservation of ISS properties under sufficiently fast sampling for nonlinear systems with 
an emulated sampled-data controller. Razumikhin's method has been used in [61] for the case of LTI sampled-data systems. In [132], the Razumikhin method is explored for nonlinear sampled-data systems on the basis of vector Lyapunov-Razumikhin functions. For more general extensions to the control design problem see [134], concerning the case of nonlinear feed-forward systems and [135], for nonlinear sampled-data system with input delays. At last, we would like to mention the Input/Output approach for the analysis of timedelay systems $[78,96,130]$, which makes use of classical robust control tools $[278,168]$. The application of the Input/Output approach for the case of sampled-data systems has been discussed in $[174,154]$. The approach was further developed by $[81,204,203,207,206,40]$ without passing through the time-delay system model. It will be presented in more detail in Section 4.4 .

\subsection{Hybrid system approach}

Due to the existence of both continuous and discrete dynamics, it is quite natural to model sampled-data systems as hybrid dynamical systems $[91,92,99]$. The first mentions to sampled-data systems as hybrid dynamical systems date back to the middle of the '80s [184]. Later on, in the '90s, the use of hybrid models has been developed for linear sampled-data systems with uniform and multi-rate sampling as an interesting approach for the $H_{\infty}$ and $H_{2}$ control problems $[126,247,256]$. The approach has also been developed for nonlinear sampleddata systems in $[115,270]$. For systems with aperiodic sampling, impulsive models had been used starting with $[256,56,170]$. Recently, more general hybrid models have been proposed in the context of Networked Controlled Systems by $[193,195]$. A solid theoretic foundation has been established for hybrid systems in the framework proposed by $[91,92]$ and it proves to be very useful in the analysis of sampled-data systems.

In this section we will present some basic hybrid models encountered in the analysis of sampled-data systems. The extensions of the Lyapunov stability theory for hybrid systems will be introduced together with constructive numerical and analytic stability analysis criteria.

\subsubsection{Impulsive models for sampled-data systems}

Consider the case of LTI sampled-data systems with linear state feedback, as in system (12). Let $\hat{x}$ denote a piecewise constant signal representing the most recent state measurement of the plant available at the controller, $\hat{x}(t)=x\left(t_{k}\right)$, for all $t \in\left[t_{k}, t_{k+1}\right), k \in \mathbb{N}$. Using the augmented system state $\chi(t)=\left[x^{T}(t), \hat{x}^{T}(t)\right]^{T} \in$ $\mathbb{R}^{n_{\chi}}$ with $n_{\chi}=2 n$, the dynamics of the LTI sampleddata system (12) can be written under the form

$$
\left\{\begin{array}{l}
\dot{\chi}(t)=F \chi(t), \quad t \neq t_{k}, k \in \mathbb{N} \\
\chi\left(t_{k}\right)=J \chi\left(t_{k}^{-}\right), \quad k \in \mathbb{N}
\end{array}\right.
$$

with

$$
\chi\left(t^{-}\right)=\lim _{\theta \uparrow t} \chi(\theta), F=\left[\begin{array}{cc}
A & B K \\
0 & 0
\end{array}\right], J=\left[\begin{array}{ll}
I & 0 \\
I & 0
\end{array}\right] .
$$

Similar models can be determined by considering an augmented state vector $\chi$ including the most recent control value implemented at the plant $\hat{u}(t)=u\left(t_{k}\right)$, the sampling error $e(t)=x(t)-\hat{x}(t)$, the actuation error $e_{u}(t)=u(t)-\hat{u}(t)$, etc. Models of the form (35),(36) fit into the framework of impulsive dynamical systems $[173,99,143,17]$ (sometimes also called discontinuous $d y$ namical systems or simply jump systems). More general nonlinear sampled-data systems lead to impulsive systems of the form $[187,193]$

$$
\begin{aligned}
& \dot{\chi}(t)=F_{k}(t, \chi(t)), \quad t \neq t_{k}, k \in \mathbb{N}, \\
& \chi\left(t_{k}\right)=J_{k}\left(t_{k}, \chi\left(t_{k}^{-}\right)\right), \quad k \in \mathbb{N},
\end{aligned}
$$

where the augmented state may also include the controller state and some of its sampled components (state, output, etc.). Generally, for an impulsive system, (37a) is called the system's flow dynamics while (37b) is the jump dynamics.

\subsubsection{Lyapunov methods for impulsive systems}

The stability of equilibria for the impulsive systems of the form (37) can be ensured by the existence of candidate Lyapunov functions that depend both on the system state and on time, and evolve in a discontinuous manner at impulse instants $[17,99,187]$.

Theorem 10 [187] Consider system (37) and denote $\tau(t)=t-t_{k}, \forall t \in\left[t_{k}, t_{k+1}\right)$. Assume that $F_{k}$ and $J_{k}$ are locally Lipschitz functions from $\mathbb{R}_{+} \times \mathbb{R}^{n_{\chi}}$ to $\mathbb{R}^{n_{\chi}}$ such that $F_{k}(t, 0)=0, J_{k}(t, 0)=0$, for all $t \geq 0$. Let there exist positive scalars $c_{1}, c_{2}, c_{3}, b$ and a Lyapunov function $V: \mathbb{R}^{n_{\chi}} \times \mathbb{R} \rightarrow \mathbb{R}$, such that

$$
c_{1}\|\chi\|^{b} \leq V(\chi, \tau) \leq c_{2}\|\chi\|^{b}
$$

for all $\chi \in \mathbb{R}^{n_{\chi}}, \tau \in[0, \bar{h}]$. Suppose that for any impulse sequence $\sigma=\left\{t_{k}\right\}_{k \in \mathbb{N}}$ such that $\underline{h} \leq t_{k+1}-t_{k} \leq \bar{h}$, $k \in \mathbb{N}$, the corresponding solution $\bar{\chi}(\cdot)$ to (37) satisfies:

$$
\frac{d V(\chi(t), \tau(t))}{d t} \leq-c_{3} V(\chi(t), \tau(t)), \quad \forall t \neq t_{k}, \forall k \in \mathbb{N},
$$

and $V\left(\chi\left(t_{k}\right), 0\right) \leq \lim _{t \rightarrow t_{k}^{-}} V(\chi(t), \tau(t)), \quad \forall k \in \mathbb{N}$. Then, the equilibrium point $\chi=0$ of system (37) is Globally Uniformly Exponentially Stable over the class of sampling impulse instants, i.e. there exist $c, \lambda>0$ such that for any sequence $\sigma=\left\{t_{k}\right\}_{k \in \mathbb{N}}$ that satisfies $\underline{h} \leq t_{k+1}-t_{k} \leq \bar{h}, k \in \mathbb{N}$

$$
\|\chi(t)\| \leq c\left\|\chi\left(t_{0}\right)\right\| e^{-\lambda\left(t-t_{0}\right)}, \quad \forall t \geq t_{0} .
$$


The previous stability theorem requires in (38) the candidate Lyapunov function to be positive at all times. For the case of system (37) with globally Lipschitz $F_{k}, k \in \mathbb{N}$, the condition can be relaxed by requiring the Lyapunov function to be positive only at impulse times [187], i.e. $c_{1}\left\|\chi\left(t_{k}\right)\right\|^{b} \leq V\left(\chi\left(t_{k}\right), 0\right) \leq c_{2}\left\|\chi\left(t_{k}\right)\right\|^{b}, \forall k \in \mathbb{N}$, instead of $(38)$.

In the case of impulsive systems (35), with linear flow and jump dynamics, candidate Lyapunov functions of the form $V(\chi, \tau)=\chi^{T} P(\tau) \chi$, with $P:[0, \bar{h}] \rightarrow \mathbb{R}^{n_{\chi} \times n_{\chi}}$ a differentiable matrix function, have been used $[257,247,27,187]$. Sufficient stability conditions can be obtained from Theorem 10 in terms of existence of a differentiable matrix function $P:[0, \bar{h}] \rightarrow \mathbb{R}^{n_{\chi} \times n_{\chi}}$, $c_{1} I \prec P(\tau) \prec c_{2} I$, satisfying the parametric set of LMIs

$$
\begin{aligned}
& F^{T} P\left(\theta_{1}\right)+P\left(\theta_{1}\right) F+c_{3} P\left(\theta_{1}\right)+\frac{\partial P}{\partial \tau}\left(\theta_{1}\right) \prec 0, \\
& \forall \theta_{1} \in[0, \bar{h}], \\
& J^{T} P(0) J-P\left(\theta_{2}\right) \prec 0, \forall \theta_{2} \in[\underline{h}, \bar{h}],
\end{aligned}
$$

with positive scalars $c_{1}, c_{2}, c_{3}$. This formulation is reminiscent of the Riccati equation approach used for robust sampled-data control in [256,247]. Alternatively, stability can also be checked by analysing the behaviour at impulse times [270,169,111,31,30,27]. The following result for impulsive systems with linear flow and jump dynamics is stated from [111].

Theorem 11 [111] Consider system (35) with $t_{k+1}-$ $t_{k} \in[\underline{h}, \bar{h}]$, for all $k \in \mathbb{N}$. The equilibrium point $\chi=0$ of system (35) is Globally Uniformly Exponentially Stable if and only if there exists a positive definite function $V_{d}$ : $\mathbb{R}^{n_{\chi}} \rightarrow \mathbb{R}_{+}$strictly convex,

$$
V_{d}(\chi)=\chi^{T} \mathcal{P}_{[\chi]} \chi
$$

homogeneous (of the second order), $\mathcal{P}_{[\cdot]}: \mathbb{R}^{n_{\chi}} \rightarrow$ $\mathbb{R}^{n_{\chi} \times n_{\chi}}, \mathcal{P}_{[\chi]}=\mathcal{P}_{[\chi]}^{T}=\mathcal{P}_{[a \chi]} \succ 0, \forall \chi \neq 0, a \in \mathbb{R}, a \neq 0$, $V(0)=0$, such that

$$
V_{d}\left(\chi\left(t_{k}\right)\right)>V_{d}\left(\chi\left(t_{k+1}\right)\right)
$$

for all $\chi\left(t_{k}\right) \neq 0, k \in \mathbb{N}$.

The result has been obtained using a linear difference inclusion $\chi\left(t_{k+1}\right) \in \mathcal{F}\left(\chi\left(t_{k}\right)\right)$ with $\mathcal{F}(\chi)=$ $\left\{J e^{F \theta} \chi, \theta \in[\underline{h}, \bar{h}]\right\}$ representing the exact system integration between two impulse instants together with converse Lyapunov theorems for linear difference inclusions $[178,114]$. More general results, concerning the impulsive systems with nonlinear dynamics, can be found in $[270,169]$. Particularizing the result of Theorem 11 to the case of quadratic Lyapunov candidate functions $V_{d}(\chi)=\chi^{T} L \chi$, sufficient stability conditions are obtained by checking the existence of a positive definite matrix $L \succ 0$ such that

$$
\left(J e^{F \theta}\right)^{T} L\left(J e^{F \theta}\right)-L \prec 0, \forall \theta \in[\underline{h}, \bar{h}],
$$

which is also a parametric LMI, similar to (39). Less conservative conditions can be obtained using composite quadratic Lyapunov functions of the form $V_{d}(\chi)=$ $\max _{i \in\{1, \ldots, M\}} \chi^{T} P_{i} \chi$, where $P_{i}, i=1, \ldots, M$, form a finite set of symmetric positive definite matrices [111].

A relation between the existence of a continuous-time Lyapunov function, $V(\chi, \tau)=\chi^{T} P(\tau) \chi$, and the existence of a quadratic Lyapunov function, $V_{d}\left(\chi\left(t_{k}\right)\right)=$ $\chi^{T}\left(t_{k}\right) L \chi\left(t_{k}\right)$, decreasing at impulse times, is provided in the following theorem.

Theorem 12 [27] The following statements are equivalent:

(a) There exists $L \succ 0$ such that

$$
\left(e^{F \theta} J\right)^{T} L\left(e^{F \theta} J\right)-L \prec 0, \forall \theta \in[\underline{h}, \bar{h}] .
$$

(b) There exists a differentiable matrix function $P$ : $[0, \bar{h}] \rightarrow \mathbb{R}^{n_{\chi} \times n_{\chi}}, P(\tau)=P^{T}(\tau), P(0) \succ 0$ and a positive scalar $\epsilon$ such that

$$
\begin{aligned}
& F^{T} P\left(\theta_{1}\right)+P\left(\theta_{1}\right) F+\frac{\partial P}{\partial \tau}\left(\theta_{1}\right) \preceq 0, \forall \theta_{1} \in[0, \bar{h}], \\
& J^{T} P(0) J-P\left(\theta_{2}\right)+\epsilon I \preceq 0, \forall \theta_{2} \in[\underline{h}, \bar{h}] .
\end{aligned}
$$

Moreover, when one of the above holds, the equilibrium $\chi=0$ of the impulsive system (35) is Asymptotically Stable for any of the possible impulse sequences $\left\{t_{k}\right\}_{k \in \mathbb{N}}$ satisfying $t_{k+1}-t_{k} \in[\underline{h}, \bar{h}]$ for all $k \in \mathbb{N}$.

Condition (b) in the previous theorem is a sufficient condition for the existence of a candidate Lyapunov function $V(\chi, \tau)=\chi^{T} P(\tau) \chi$ such that

$$
\frac{d V(\chi(t), \tau(t))}{d t} \leq 0, \quad \forall t \neq t_{k}, \forall k \in \mathbb{N}
$$

and

$$
V\left(\chi\left(t_{k}\right), 0\right)<\lim _{t \rightarrow t_{k}^{-}} V(\chi(t), \tau(t)), \quad \forall k \in \mathbb{N},
$$

which is slightly different from the conditions (39) obtained based on Theorem 10. For the case described in Theorem 12 , (b), $V(\chi, \tau)$ may be constant between impulse instants provided that it decays at impulse times. According to the previous theorem, the existence of such a candidate Lyapunov function is equivalent to the existence of a quadratic Lyapunov function $V_{d}(\chi)=\chi^{T} L \chi$ 
which is strictly decreasing at impulse times. Note that from Theorem 11, the latter is only a sufficient condition for stability. The main point is that the existence of a function of the form $V(\chi, \tau)=\chi^{T} P(\tau) \chi$ satisfying $(43),(44)$ is not a necessary condition for the stability of the impulsive system (35). Other forms of Lyapunov functions need to be considered to improve the existing conditions.

\subsubsection{Numerically tractable stability criteria}

In practice, the difficulty of checking the existence of candidate Lyapunov functions using LMI formulations such as (39) or (41) comes from the fact that the set of LMIs are parametrized by elements in $[0, \bar{h}]$ or $[\underline{h}, \bar{h}]$, which leads to an infinite number of LMIs. As follows we will discuss the derivation of a finite number of LMIs from (39). Numerical tools $[111,31,30]$ for the resolution of LMIs similar to (41), involving uncertain matrix exponential terms, are discussed in Section 4.3 in the context of the discrete-time approach.

Concerning the parametric set of LMIs (39), a finite number of LMI conditions can be derived by considering particular forms for the matrix function $P(\tau)$. For example, consider a matrix $P(\tau)$ linear with respect to $\tau$

$$
P(\tau)=P_{1}+\left(P_{2}-P_{1}\right) \frac{\tau}{\bar{h}}
$$

for some positive definite matrices $P_{1}, P_{2}$, as in $[120,4]$. There, such a Lyapunov matrix has been used for sampled-data systems with multi-rate sampling and switched linear systems. For a candidate Lyapunov function $V(\chi, \tau)=\chi^{T} P(\tau) \chi$, with $P(\tau)$ as defined in (45), a finite set of LMIs that are sufficient for stability can be obtained from (39) using simple convexity arguments:

$$
\begin{aligned}
& F^{T} P_{1}+P_{1} F+c_{3} P_{1}+\frac{P_{2}-P_{1}}{\bar{h}} \prec 0, \\
& F^{T} P_{2}+P_{2} F+c_{3} P_{2}+\frac{P_{2}-P_{1}}{\bar{h}} \prec 0, \\
& J^{T} P_{1} J \prec P_{2}, \\
& J^{T} P_{1} J \prec P_{1}+\left(P_{2}-P_{1}\right) \underline{h} / \bar{h} .
\end{aligned}
$$

For the particular case of LTI sampled-data systems represented by $(35),(36)$, Lyapunov functions of the form $V(\chi, \tau)=\chi^{T} P(\tau) \chi$ are proposed in the literature by summing various terms such as:

$$
\begin{aligned}
& V_{1}(\chi, \tau)=x^{T} P_{0} x \\
& V_{2}(\chi, \tau)=(x-\hat{x})^{T} Q(x-\hat{x})(\bar{h}-\tau) \\
& V_{3}(\chi, \tau)=(x-\hat{x})^{T} R(x-\hat{x}) e^{-\lambda \tau} \\
& V_{4}(\chi, \tau)=e^{-\lambda \tau} \chi^{T}\left(e^{F^{T}(\bar{h}-\tau)} S e^{F^{T}(\bar{h}-\tau)}\right) \chi \\
& V_{5}(\chi, \tau)=\chi^{T}\left(\int_{-\tau}^{0}(s+\bar{h})\left(F e^{F s}\right)^{T} \tilde{U}\left(F e^{F s}\right) d s\right) \chi
\end{aligned}
$$

where $\quad \tilde{U}:=\left[\begin{array}{ll}U & 0 \\ 0 & 0\end{array}\right], \lambda>0$ and $P_{0}, Q, R, S, U$ are symmetric positive definite matrices. Using such particular forms of Lyapunov functions, LMI stability conditions have been derived in the literature $[120,187,195,205,92,65]$. We point in particular to the term (51) used in [187] which provided a significant improvement in what concerns the conservatism reduction. This term is inspired by Lyapunov-Krasovskii functionals from the input-delay approach, like the one in [72]. Note that the term (51) can also be written as $\int_{t-\tau}^{t}(s+\bar{h}-t) \dot{x}^{T}(s) U \dot{x}(s) d s$. It has been motivated by the term $\int_{-\bar{h}}^{0} \int_{t+\theta}^{t} \dot{x}^{T}(s) U \dot{x}(s) d s d \theta$ used in the timedelay approach (see [72]). Vice versa, the hybrid system approach has also inspired the use of discontinuous Lyapunov functionals in the time-delay approach (see for example the functional (29)). Note that the term $\left(h_{k}-\tau\right) \int_{t-\tau}^{t} \dot{x}^{T}(s) R \dot{x}(s) d s$ in the functional (29) can be re-written as $\left(h_{k}-\tau\right) \chi^{T}\left(\int_{-\tau}^{0}\left(F e^{F s}\right)^{T} \tilde{R}\left(F e^{F s}\right) d s\right) \chi$, with

$$
\tilde{R}=\left[\begin{array}{ll}
R & 0 \\
0 & 0
\end{array}\right]
$$

and $R \succ 0$. Then, for the impulsive system (35), (36), the functional (29) can be interpreted as a Lyapunov function of the form $V\left(\chi, \tau, h_{k}\right)=\chi^{T} P\left(\tau, h_{k}\right) \chi$. Similarly to the time delay approach, the LMI formulations can be adapted to cope with uncertainties in the system matrices.

Last, note that alternatively to the LMI formulation, numerical stability criteria based on polynomial matrix functions $P(\tau)$ and Sum-of-Squares programming [222] have been proposed in [27].

\subsubsection{More general hybrid models}

A large variety of hybrid dynamical systems, including sampled-data and impulsive models, can be reformulated in the unifying theoretical framework proposed by Goebel, Sanfelice and Teel $[91,92]$. Several fundamental properties have been investigated in this framework, providing a solid theory for hybrid dynamical systems. The main advantage of this generic hybrid formulation $[91,92]$ is that the associated theoretic properties can be directly transferred to sampled-data systems with aperiodic sampling. The general formulation proposed in $[91,92]$ considers models of the form

$$
\begin{aligned}
& \dot{z}=F_{z}(z), \quad z \in C, \\
& z^{+}=J_{z}(z), \quad z \in D,
\end{aligned}
$$

with state $z \in \mathbb{R}^{n_{z}}$. The system state evolves according to an ordinary differential equation (52a) when the state is in some subset $C$ of $\mathbb{R}^{n_{z}}$ and according to a first order 
recurrence equation $(52 \mathrm{~b})$ when the state is in the subset $D$ of $\mathbb{R}^{n_{z}} \cdot z^{+}$denotes the next value of state given as a function of the current state $z$ via the map $J_{z}(\cdot) . C$ is called the flow set and $D$ is called the jump set. Here, we assume that $F_{z}$ and $J_{z}$ are continuous functions from $C$ to $\mathbb{R}^{n_{z}}$ and $D$ to $\mathbb{R}^{n_{z}}$, respectively. $C$ and $D$ are assumed to be closed sets in $\mathbb{R}^{n_{z}}$.

Note that in the impulsive system formulation of sampled-data systems, the system jumps are timetriggered. However, the dynamics of the triggering mechanism is in some sense hidden. In the framework proposed by $[35,46,195,91,92]$, the mechanism triggering the system jumps is modelled explicitly by augmenting the system state with the clock variable $\tau(t)=t-t_{k}$, $\forall t \in\left[t_{k}, t_{k+1}\right), \forall k \in \mathbb{N}$. Consider the LTI sampleddata systems (12) with the notations $\hat{x}(t)=x\left(t_{k}\right)$, $\tau(t)=t-t_{k}$ for all $t \in\left[t_{k}, t_{k+1}\right), k \in \mathbb{N}$. The system can be represented by the following hybrid model

$$
\left\{\begin{array}{l}
\dot{x}=A x+B K \hat{x} \\
\dot{\hat{x}}=0 \\
\dot{\tau}=1 \\
x^{+}=x \\
\hat{x}^{+}=x \\
\tau^{+}=0
\end{array}\right\} \quad \tau \in[0, \bar{h}],
$$

Then, system (12) with $h_{k} \in[\underline{h}, \bar{h}]$ (or equivalently $(35),(36))$ can be re-modelled in the form (52) with $z^{T}=\left[\begin{array}{lll}x^{T} & \hat{x}^{T} & \tau\end{array}\right]=\left[\begin{array}{ll}\chi^{T} & \tau\end{array}\right]$

$$
\begin{gathered}
C=\left\{z \in \mathbb{R}^{n_{z}}: \tau \in[0, \bar{h}]\right\} \\
D=\left\{z \in \mathbb{R}^{n_{z}}: \tau \in[\underline{h}, \bar{h}]\right\} \\
F_{z}(z)=\left[\begin{array}{c}
A x+B K \hat{x} \\
0 \\
1
\end{array}\right], J_{z}(z)=\left[\begin{array}{l}
x \\
x \\
0
\end{array}\right] .
\end{gathered}
$$

Solutions $\phi$ of the general hybrid system (52) are parametrized by both the continuous time $t$ and the discrete time $k: \phi(t, k)$ represents the state of the hybrid system after $t$ time units and $k$ jumps. Such solutions are defined on a hybrid time domain, which for the case of sampled-data systems is given as the union of the intervals $\left[t_{k}, t_{k+1}\right] \times\{k\}$. A solution $\phi(\cdot, \cdot)$ is a function defined on a hybrid time domain such that $\phi(\cdot, k)$ is continuous on $\left[t_{k}, t_{k+1}\right]$, continuously differentiable on $\left(t_{k}, t_{k+1}\right)$ for each $k$ in the domain, and such that

$$
\dot{\phi}(t, k)=F_{z}(\phi(t, k)),
$$

if $\phi(t, k) \in C, t \in\left(t_{k}, t_{k+1}\right), k \in \mathbb{N}$, and

$$
\phi\left(t_{k+1}, k+1\right)=J_{z}\left(\phi\left(t_{k+1}, k\right)\right),
$$

if $\phi\left(t_{k+1}, k\right) \in D, k \in \mathbb{N}$. For sampled-data systems as (53) such solutions may be roughly interpreted as a generalization of the state lifting approach proposed in [269] for systems with periodic sampling.

A particularity of the model (53) in the context of stability analysis is the fact that although the matrix $K$ is designed such that $x$ (and consequently $\hat{x}$ ) converges to zero, the clock variable $\tau$ does not converge. For each sampling interval $\left[t_{k}, t_{k+1}\right)$, the timer $\tau$ visits successively the points of the interval $[0, \bar{h}]$ up to $h_{k}=t_{k+1}-$ $t_{k}$. The main consequence is that the hybrid system (53) does not have an asymptotically stable equilibrium point. For such systems the stability of the compact set $\mathcal{A}=\{0\} \times\{0\} \times[0, \bar{h}]$ is usually investigated instead. Studying this property allows to conclude on the convergence of $x$. One of the main results allowing to state the asymptotic stability of a set for hybrid systems is given below. This results is expressed in terms of the pre-asymptotic stability of a set $\mathcal{A}$ (see [91] for a detailed definition). The prefix "-pre" is used since the completeness of all system solutions ${ }^{2}$ is not required. Only complete solutions need to converge to $\mathcal{A}$. The concept of pre-asymptotic stability used in the following theorem is equivalent to standard asymptotic stability of the set $\mathcal{A}$ when all system solutions are complete, which is the case for sampled-data systems.

Theorem 13 [91] Consider the hybrid system (52) and the compact set $\mathcal{A} \subset \mathbb{R}^{n_{z}}$ such that $J_{z}(\mathcal{A} \cap D) \subset \mathcal{A}$. If there exists a candidate Lyapunov function ${ }^{3} V$ such that

$$
\begin{aligned}
& \frac{\partial V}{\partial z} F_{z}(z)<0 \text { for all } z \in C \backslash \mathcal{A}, \\
& V\left(J_{z}(z)\right)-V(z)<0 \text { for all } z \in D \backslash \mathcal{A},
\end{aligned}
$$

then the set $\mathcal{A}$ is pre-asymptotically stable.

Various relaxations of the above result are provided in Chapter 3 in [92]. A converse Lyapunov theorem is given below.

Theorem 14 [91] For the hybrid system (52), if the compact set $\mathcal{A}$ is globally pre-asymptotically stable, then there exist a $\mathcal{C}^{\infty}$ function $V: \mathbb{R}^{n_{z}} \rightarrow \mathbb{R}_{+}$and $\alpha_{1}, \alpha_{2} \in$ $\mathcal{K}_{\infty}$ such that $\alpha_{1}\left(|z|_{\mathcal{A}}\right) \leq V(z) \leq \alpha_{2}\left(|z|_{\mathcal{A}}\right), \forall z \in \mathbb{R}^{n_{z}}$, where $|\cdot|_{\mathcal{A}}$ denotes the distance from the set $\mathcal{A}$, and

$$
\begin{aligned}
\frac{\partial V}{\partial z} F_{z}(z) & \leq-V(z), \forall z \in C, \\
V\left(J_{z}(z)\right) & \leq V(z) / 2, \forall z \in D .
\end{aligned}
$$

\footnotetext{
${ }^{2}$ A solution $\phi(t, k)$ is called complete if dom $\phi$ is unbounded. ${ }^{3} V$ is continuous and non-negative on $(C \cup D) \backslash \mathcal{A} \subset \operatorname{dom} V$, it is continuously differentiable on an open set satisfying $C \backslash \mathcal{A} \subset \operatorname{dom} V$, and $\lim _{z \rightarrow \mathcal{A}, z \in \operatorname{dom} V \cap(C \cup D)} V(z)=0$. Furthermore, for global pre-asymptotic stability, the sublevel sets of $V($.$) are required to be compact.$
} 
Note that with respect to the case of sampled-data systems such as (53) (or equivalently (35), (36)) where solutions are complete, the previous theorem shows that asymptotic stability implies the existence of a $C^{\infty}$ Lyapunov function of the form $V(z)=\tilde{V}(\chi, \tau)$, to be related with the sufficient conditions for stability in Theorem 10 .

\subsubsection{An estimation of the MSI for nonlinear systems}

For nonlinear sampled-data systems the stability properties have been studied in the more general context of Networked Control Systems with scheduling protocols $[193,35]$. This approach has been particularized to the sampled-data case in [195]. Consider the plant:

$$
\left\{\begin{array}{l}
\dot{x}=F(x, u) \\
y=H(x, u)
\end{array}\right.
$$

where $x$ is the plant state, $u$ is the control input, $y$ is the measured output. Suppose that asymptotic stability is guaranteed by the continuous-time output feedback:

$$
\left\{\begin{array}{l}
\dot{x}^{c}=F^{c}\left(x^{c}, y\right), \\
u=H^{c}\left(x^{c}, y\right),
\end{array}\right.
$$

where $x^{c}$ is the controller state. Under an exact sampleddata implementation of the controller and a perfect knowledge of the sampling sequence $\sigma=\left\{t_{k}\right\}_{k \in \mathbb{N}}$, the sampled-data implementation of the closed-loop system can be written in the following impulsive form:

$$
\left\{\begin{array}{rlrl}
\dot{x} & =F(x, \hat{u}), & & t \in\left[t_{k}, t_{k+1}\right), \\
y & =H(x), & t \in \mathbb{R}_{+}, \\
\dot{x}^{c} & =F^{c}\left(x^{c}, \hat{y}\right), & t \in\left[t_{k}, t_{k+1}\right), \\
u & =H^{c}\left(x^{c}\right), & t \in \mathbb{R}_{+}, \\
\dot{\hat{y}}=0, & & t \in\left[t_{k}, t_{k+1}\right), \\
\dot{\hat{u}} & =0, & t \in\left[t_{k}, t_{k+1}\right), \\
\hat{y}\left(t_{k}\right) & =y\left(t_{k}^{-}\right), & & \\
\hat{u}\left(t_{k}\right) & =u\left(t_{k}^{-}\right), & &
\end{array}\right.
$$

where $\hat{u}$ represents the control being implemented at the plant and $\hat{y}$ the most recent plant output measurements that are available at the controller. In order to express the system in the general framework of [92], consider the augmented state vector $\eta(t) \in \mathbb{R}^{n_{\eta}}$ and the samplinginduced error $e(t) \in \mathbb{R}^{n_{e}}$ :

$$
\eta(t):=\left[\begin{array}{c}
x(t) \\
x^{c}(t)
\end{array}\right], e(t)=\left[\begin{array}{l}
e^{y}(t) \\
e^{u}(t)
\end{array}\right]:=\left[\begin{array}{l}
\hat{y}(t)-y(t) \\
\hat{u}(t)-u(t)
\end{array}\right]
$$

The dynamics in (59) with $h_{k} \in[\underline{h}, \bar{h}]$ can be modelled by the following hybrid system:

$$
\left\{\begin{array}{l}
\dot{\eta}=f(\eta, e) \\
\dot{e}=g(\eta, e) \\
\dot{\tau}=1 \\
\eta^{+}=\eta \\
e^{+}=0 \\
\tau^{+}=0
\end{array}\right\} \quad \tau \in[0, \bar{h}],
$$

with $\eta \in \mathbb{R}^{n_{\eta}}, e \in \mathbb{R}^{n_{e}}, \tau \in \mathbb{R}_{+}$. The functions $f$ and $g$ are obtained by direct calculations from the sampleddata system (59) (see [195]):

$$
\begin{gathered}
f(\eta, e)=\left[\begin{array}{c}
F\left(x, H^{c}\left(x^{c}\right)+e^{u}\right) \\
F^{c}\left(x^{c}, H(x)+e^{y}\right)
\end{array}\right], \\
g(\eta, e)=\left[\begin{array}{c}
-\frac{\partial H}{\partial x} F\left(x, H^{c}\left(x^{c}\right)+e^{u}\right) \\
-\frac{\partial H^{c}}{\partial x^{c}} F^{c}\left(x^{c}, H(x)+e^{y}\right)
\end{array}\right] .
\end{gathered}
$$

It should be noted that $\dot{\eta}=f(\eta, 0)$ is the closed loop system without the sampled-data implementation. The following theorem provides a quantitative method to estimate the MSI, using model (60).

Theorem 15 [195] Assume that $f$ and $g$ in (60) are continuous. Suppose there exist $\tilde{\Delta}_{\eta}, \tilde{\Delta}_{e}>0$, a locally Lipschitz function $W: \mathbb{R}^{n_{e}} \rightarrow \mathbb{R}_{+}$, a locally Lipschitz, positive definite, radially unbounded function $V: \mathbb{R}^{n_{\eta}} \rightarrow \mathbb{R}_{+}$, a continuous function $\Theta: \mathbb{R}^{n_{\eta}} \rightarrow \mathbb{R}_{+}$, real numbers $L>0, \gamma>0$, functions $\underline{\alpha}_{W}, \bar{\alpha}_{W} \in \mathcal{K}_{\infty}$ and a continuous, positive definite function @ such that, for all $e \in \mathbb{R}^{n_{e}}$ :

$$
\underline{\alpha}_{W}(\|e\|) \leq W(e) \leq \bar{\alpha}_{W}(\|e\|)
$$

and for almost all $\|\eta\| \leq \tilde{\Delta}_{\eta}$ and $\|e\| \leq \tilde{\Delta}_{e}$ :

$$
\begin{gathered}
\frac{\partial W}{\partial e} g(\eta, e) \leq L W(e)+\Theta(\eta), \\
\frac{\partial V}{\partial \eta} f(\eta, e) \leq-\varrho(\|\eta\|)-\varrho(W(e))-\Theta^{2}(\eta)+\gamma^{2} W^{2}(e) .
\end{gathered}
$$

Finally, consider that $0<\underline{h} \leq \bar{h}<\mathcal{T}(\gamma, \mathcal{L})$, with

$$
\mathcal{T}(\gamma, L):= \begin{cases}\frac{1}{\operatorname{Lr}} \arctan (r), & \gamma>L \\ \frac{1}{L}, & \gamma=L \\ \frac{1}{L r} \operatorname{arctanh}(r), & \gamma<L\end{cases}
$$

and $r=\sqrt{\left|\frac{\gamma^{2}}{L^{2}}-1\right|}$. Then, for all sampling intervals less than $\bar{h}$ the set $\mathcal{A}=\{(\eta, e, \tau): \eta=0, e=0, \tau \in[0, \bar{h}]\}$ is Uniformly Asymptotically Stable for system (60). 
Theorem 15 provides an explicit formulation of the MSI for nonlinear sampled-data systems. It is applicable for both constant and variable sampling intervals. For a constructive application to the case of bilinear systems see [205]. A numerical formulation using Sum-of-Squares [222] has also been provided in [22].

\subsubsection{Further reading}

In the impulsive system framework, control design conditions have been proposed in [27]. For observer design conditions we point to the works in $[5,49,3,186,220,59]$. Some extensions of the hybrid systems approach for sampleddata systems with delay can be found in $[73,188]$ and $[22]$. When constructing a hybrid model for linear sampleddata system, it is also possible to consider as state variable a decreasing counter $\theta(t)=h_{k}-\tau(t)$ with $\dot{\theta}=-1$. Aside from the methodology presented here, the stability of impulsive systems can also be analysed using discrete-time approaches [111] based on convex embedding methods and looped Lyapunov functionals $[31,30]$. These methods are presented for the particular case of sampled-data systems in Section 4.3.

\subsection{Discrete-time approach and convex-embeddings}

In this sub-section we present several approaches which use either the system integration over the sampling interval or convex embeddings of the transition matrix between sampling times in order to derive stability conditions. We will start with extensions of the discrete-time approach for the case of linear systems with aperiodic sampling; next a technique based on Delta operators will be given, followed by a continuous-time approach and a discrete-time approach based on functionals, similar to the ones used in the time-delay approach. At last, an extension of the discrete-time approach to nonlinear systems with aperiodic sampling will be presented.

\subsubsection{Theoretical results for LTI systems using the discrete-time approach}

Let us consider the LTI system with sampled linear static state feedback (12) where $h_{k}=t_{k+1}-t_{k}$ takes values in the set $\mathcal{T}=[\underline{h}, \bar{h}]$. Recall the notations $x_{k}=x\left(t_{k}\right)$,

$$
\Lambda(\theta)=\mathrm{e}^{A \theta}+\int_{0}^{\theta} \mathrm{e}^{A s} d s B K
$$

for $\theta \in \mathbb{R}$. One can verify that the closed-loop system (12) satisfies

$$
x_{k+1}=\Lambda\left(h_{k}\right) x_{k}
$$

with $h_{k} \in \mathcal{T}=[\underline{h}, \bar{h}]$. Model (62) belongs to the class of discrete-time Linear Parameter Varying (LPV) systems $[227,127,178]$. It captures the behaviour of system (12) at sampling times, without consideration of the intersample behavior. However, in [81], the following proposition has shown that for LTI sampled-data system, the asymptotic stability in continuous-time and in discretetime are equivalent.

Proposition 16 [81] Consider the sampled-data system (12) with $h_{k}=t_{k+1}-t_{k} \in[\underline{h}, \bar{h}]$. For a given $x\left(t_{0}\right)$, the following conditions are equivalent:

(1) $\lim _{t \rightarrow \infty} x(t)=0$

(2) $\lim _{k \rightarrow \infty} x\left(t_{k}\right)=0$.

Various methods are available for studying the stability of discrete-time LPV systems. Stability criteria have been proposed by analysing the joint spectral radius $[24,1]$ or by checking the existence of quasiquadratic [178,121], parameter dependent [45], pathdependent [144], non-monotonic [167,141,2] and composite quadratic [121] Lyapunov functions.

The following theorem from [114] addresses specifically the case of model (62).

Theorem 17 [114] Consider the continuous-time system (12) and the discrete-time model (62) with $\mathcal{T}=$ $[\underline{h}, \bar{h}]$. The following statements are equivalent:

1) The equilibrium point $x=0$ of (62) is Globally Uniformly Exponentially Stable.

2) There exist a $P \succ 0$ and $N>0$ such that

$$
\left(\prod_{i=1}^{N} \Lambda\left(\theta_{i}\right)\right)^{T} P\left(\prod_{i=1}^{N} \Lambda\left(\theta_{i}\right)\right)-P \prec 0
$$

for any $N$-length sequence $\left\{\theta_{i}\right\}_{i=1}^{N}$ with values in $\mathcal{T}$, i.e. the function $\bar{V}(x)=x^{T}$ Px satisfies $\bar{V}\left(x_{k+N}\right)<\bar{V}\left(x_{k}\right)$ for all $x_{k} \neq 0, k \in \mathbb{N}$.

3) There exists a positive definite function $V: \mathbb{R}^{n} \rightarrow$ $\mathbb{R}^{+}$strictly convex, homogeneous (of the second order), $V(x)=x^{T} \mathcal{P}_{[x]} x$, with $\mathcal{P}_{[\cdot]}: \mathbb{R}^{n} \rightarrow \mathbb{R}^{n \times n}, \mathcal{P}_{[x]}=\mathcal{P}_{[x]}^{T}=$ $\mathcal{P}_{[a x]}, \forall x \neq 0, a \in \mathbb{R}, a \neq 0$ such that :

$$
V(x)-\max _{\theta \in \mathcal{T}} V(\Lambda(\theta) x)>0, \forall x \neq 0
$$

Condition 2) in Theorem 17 corresponds to the existence of a non-monotonic Lyapunov function $\bar{V}(x)=x^{T} P x$, $[167,141,2]$ which is decreasing every $N$ samples. If the system is stable, then necessarily there exists a finite $N$ and a matrix $P$ such that (63) holds. However, checking the existence of a matrix $P$ satisfying (63) for a given $N$ represents a set of LMIs which are sufficient only for stability. Condition 3) corresponds to the existence of 
a quasi-quadratic Lyapunov function $[178,121] V(x)=$ $x^{T} \mathcal{P}_{[x]} x$. Theorem 17 shows the equivalence between quasi-quadratic Lyapunov functions and non-monotonic Lyapunov functions and provides necessary and sufficient conditions for the exponential stability of system (62). A simple stability criterion which is sufficient for stability can be obtained using classical quadratic Lyapunov functions, which are decreasing at each sample.

Theorem 18 [275] The origin of system (62) is Globally Uniformly Exponentially Stable for all sampling sequences $\sigma=\left\{t_{k}\right\}_{k \in \mathbb{N}}$ with $h_{k}=t_{k+1}-t_{k} \in[\underline{h}, \bar{h}], k \in \mathbb{N}$, if there exists $P \succ 0$ such that

$$
\Lambda^{T}(\theta) P \Lambda(\theta)-P \prec 0, \forall \theta \in \mathcal{T}=[\underline{h}, \bar{h}] .
$$

The LMI (65) is a particular case of condition 2) in Theorem 17 with $N=1$. In a similar way, it is also a particular case of condition 3 ) with $\mathcal{P}_{[x]}=P$, for all $x \in \mathbb{R}^{n}$. The condition ensures that the candidate Lyapunov function $V(x)=x^{T} P x$ satisfies the relation

$$
\Delta V(k)=V\left(x_{k+1}\right)-V\left(x_{k}\right)<0, \forall x_{k} \neq 0 .
$$

Finally, let us note that, similarly to conditions (39) or (41) used for the hybrid system approach, the stability conditions (63) and (65) represent sets of LMIs that are parametrized by $\theta \in \mathcal{T}=[\underline{h}, \bar{h}]$. They are not computationally tractable problems by themselves. Approximate solutions, based on evaluation of the condition for a finite set of values of $\theta$ have been presented in [275,228,241]. A finite set of sufficient tractable numerical conditions can be obtained using normed-bounded and/or polytopic convex embeddings of the transition matrix $\Lambda(\theta)$.

\subsubsection{Tractable criteria}

In what follows, we try to give an idea about the manner to solve parametric LMIs involving matrix exponentials such as the one in (65). First, we present briefly the approach proposed by Fujioka in [79]. Consider a nominal sampling interval $T_{0} \in[\underline{h}, \bar{h}]$. For a scalar $\delta$, the transition matrix $\Lambda(\cdot)$ satisfies the relation

$$
\Lambda\left(T_{0}+\delta\right)=\Lambda\left(T_{0}\right)+\Delta(\delta) \Psi\left(T_{0}\right)
$$

where $\Delta(\delta):=\int_{0}^{\delta} e^{A s} d s, \Psi\left(T_{0}\right)=A \Lambda\left(T_{0}\right)+B K$. Using classical properties of the matrix exponential [155], the induced Euclidean norm of $\Delta(\delta)$ can be over-bounded

$$
\|\Delta(\delta)\|_{2} \leq \int_{0}^{\delta} e^{\mu(A) s} d s
$$

where $\mu(A)$ is the maximum eigenvalue of $\frac{A+A^{T}}{2}$. System (62) can be expressed as a nominal discrete-time LTI system with a norm-bounded uncertainty

$$
x_{k+1}=\Lambda\left(T_{0}\right) x_{k}+\Delta\left(\delta_{k}\right) \Psi\left(T_{0}\right) x_{k}
$$

where $\delta_{k}=h_{k}-T_{0}$, for which classical $H_{\infty}$ criteria [86] can be used. A simplified version of the main result in $[79]$ is given as follows.

Theorem 19 [79] Let $T_{0}>0$ be given. If there exist $X \succ 0$ and $\gamma>0$ satisfying

$$
\begin{aligned}
& \mathcal{M}\left(T_{0}, X, \gamma\right):=
\end{aligned}
$$

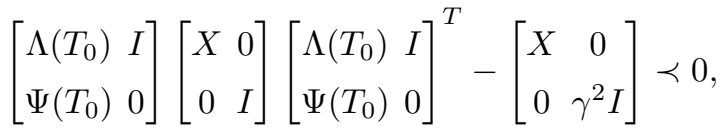

then (65) is satisfied with $P=X^{-1}$ for all $\theta \in$ $\mathcal{T}\left(T_{0}, \gamma\right):=\left[\underline{h}\left(T_{0}, \gamma\right), \bar{h}\left(T_{0}, \gamma\right)\right] \cap(0, \infty)$, with

$$
\begin{aligned}
& \underline{h}\left(T_{0}, \gamma\right)=\left\{\begin{array}{l}
T_{0}-\gamma^{-1}, \text { if } \mu(-A)=0, \\
-\infty, \text { if } \mu(-A) \leq-\gamma, \\
T_{0}-\frac{\log \left(1+\gamma^{-1} \mu(-A)\right)}{\mu(-A)}, \text { otherwise, }
\end{array}\right. \\
& \bar{h}\left(T_{0}, \gamma\right)=\left\{\begin{array}{l}
T_{0}+\gamma^{-1}, \text { if } \mu(A)=0, \\
\infty, \text { if } \mu(A) \leq-\gamma, \\
T_{0}+\frac{\log \left(1+\gamma^{-1} \mu(A)\right)}{\mu(A)}, \text { otherwise. }
\end{array}\right.
\end{aligned}
$$

Condition (70) is sufficient for the asymptotic stability of system (62) under time-varying sampling intervals $h_{k} \in$ $[\underline{h}, \bar{h}]$ with $\underline{h}$ and $\bar{h}$ given in (71) and (72), respectively. Other norm-bounded approximations of the transition matrix $\Lambda(\cdot)$ exist in the literature $[18,245,129,85,277]$. For example, stability conditions have been provided using the Schur decomposition in [245] while [277] uses the Jordan normal form. In [85] the transition matrix $\Lambda\left(T_{0}+\delta\right)$ is decomposed as

$$
\Lambda\left(T_{0}+\delta\right)=\Lambda\left(T_{0}\right)+\delta L\left(T_{0}\right)+\Delta_{2}(\delta) A L\left(T_{0}\right)
$$

with $L\left(T_{0}\right)=e^{A T_{0}}(A+B K), \Delta_{2}(\delta):=\int_{0}^{\delta} \int_{0}^{\rho} e^{A s} d s d \rho$, and stability conditions are provided by computing the induced Euclidean norm of $\Delta_{2}(\delta)$. See also [129] where stability conditions have been derived using Integral Quadratic Constraints (IQC), by studying the positive realness of $\Delta(\delta)$. More general Lyapunov functions have been used in [84].

Alternatively to the use of norm bounded approximations, tractable numerical conditions can also be obtained using polytopic embeddings of the transition matrix $\Lambda(\cdot)$ in system (62). The set

$$
\mathcal{W}_{[\underline{h}, \bar{h}]}:=\{\Lambda(\theta), \theta \in[\underline{h}, \bar{h}]\}
$$


is embedded in a larger convex polytope with a finite number of vertices $\Lambda_{i}, i \in \mathcal{I}:=\left\{1, \cdots, N_{v}\right\}$,

$$
\overline{\mathcal{W}}:=\left\{\sum_{i=1}^{N_{v}} \alpha_{i} \Lambda_{i} \mid \alpha_{i} \geq 0, i \in \mathcal{I}, \sum_{i=1}^{N_{v}} \alpha_{i}=1\right\}
$$

in such a way that $\mathcal{W}_{[\underline{h}, \bar{h}]} \subseteq \overline{\mathcal{W}}$. Using a polytopic embedding, system (62) can be expressed as a

$$
x_{k+1}=\sum_{i=1}^{N_{v}} \alpha_{i}\left(h_{k}\right) \Lambda_{i} x_{k}
$$

where $\sum_{i=1}^{N_{v}} \alpha_{i}\left(h_{k}\right)=1, \alpha_{i}\left(h_{k}\right) \geq 0, i \in \mathcal{I}$. This is a classical discrete-time system with polytopic uncertainty [45]. Here $\alpha\left(h_{k}\right)=\left[\alpha_{1}\left(h_{k}\right) \alpha_{2}\left(h_{k}\right) \ldots \alpha_{N_{v}}\left(h_{k}\right)\right]^{T}$ represent the barycentric coordinates of $\Lambda\left(h_{k}\right)$ in the polytope $\overline{\mathcal{W}}$. The properties of the over-approximating polytopic set $\overline{\mathcal{W}}$ make it possible to derive a finite number of sufficient stability conditions from (65), by writing simple LMIs over the polytope vertices:

$$
P \succ 0, \Lambda_{i}^{T} P \Lambda_{i}-P \prec 0, \forall i \in \mathcal{I} \text {. }
$$

The same procedure can also be applied for the condition (63) - see [114] for details. However, for the case of conditions (63), the numerical complexity is increasing in an exponential manner with respect to the chosen parameter $N$. One of the advantages of the polytopic embedding is the fact that it allows the use of parameter dependent Lyapunov functions $[45,108,43] \tilde{V}(x, \alpha)=$ $x^{T} P(\alpha) x, P(\alpha)=\sum_{i=1}^{N_{v}} \alpha_{i} P_{i}$, which lead to refined stability conditions under a reasonable numerical complexity:

$$
\exists P_{i}=P_{i}^{T} \succ 0, \Lambda_{i}^{T} P_{j} \Lambda_{i}-P_{i} \prec 0, \forall(i, j) \in \mathcal{I} \times \mathcal{I} .
$$

With respect to Theorem 17 , the set of conditions (76) represents also a sufficient criterion for the existence of a quasi-quadratic Lyapunov function [111], $V(x)=$ $x^{T} \mathcal{P}_{[x]} x=\max _{i \in\left\{1, \ldots, N_{v}\right\}} x^{T} P_{i} x$.

The main difficulty in constructing the polytope $\overline{\mathcal{W}}$ is the exponential dependence of the transition matrix $\Lambda(\theta)=$ $e^{A \theta}+\int_{0}^{\theta} e^{A s} d s B K$ in the parameter $\theta$ over the the interval $[\underline{h}, \bar{h}]$. Several approaches exist for the computation of a convex polytope embedding an uncertain matrix exponential. See for example $[201,200,42,43,157]$ for techniques based on the real Jordan form, [90] for a construction that uses the Cayley-Hamilton theorem and [41] for an approach studying interval matrices. One may remark that the transition matrix $\Lambda(\cdot)$ can be re-expressed as

$$
\Lambda(\theta)=I+\Delta(\theta)(A+B K)
$$

which involves only one uncertain matrix term $\Delta(\theta)=$ $\int_{0}^{\theta} e^{A s} d s$. Then the stability problem can be addressed by constructing a polytopic approximation of $\Delta(\theta)$ for $\theta \in[\underline{h}, \bar{h}]$. To give an idea about the manner such a convex polytope can be constructed, let us consider a simple case where the matrix $A$ has $n$ real eigenvalues $\lambda_{i} \neq 0, i \in\{1, \ldots, n\}$ with multiplicity equal to one, i.e.

$$
A=T^{-1}\left[\begin{array}{ccccc}
\lambda_{1} & 0 & 0 & \ldots & 0 \\
0 & \lambda_{2} & 0 & \ldots & 0 \\
\vdots & & \ddots & & \vdots \\
0 & \ldots & \ldots & 0 & \lambda_{n}
\end{array}\right] T
$$

for some invertible matrix $T \in \mathbb{R}^{n \times n}$. Then the uncertain matrix $\Delta(\theta)$ takes the form:

$$
\Delta(\theta)=T^{-1}\left[\begin{array}{ccccc}
\rho_{1}(\theta) & 0 & 0 & \ldots & 0 \\
0 & \rho_{2}(\theta) & 0 & \ldots & 0 \\
\vdots & & \ddots & & \vdots \\
0 & \ldots & \ldots & 0 & \rho_{n}(\theta)
\end{array}\right] T
$$

where $\rho_{i}(\theta)=\frac{1}{\lambda_{i}}\left(e^{\lambda_{i} \theta}-1\right), i=1, \ldots, n$. By computing $\rho_{i}^{\text {min }}$ and $\rho_{i}^{\text {max }}$ the minimum and maximum values of $\rho_{i}(\theta)$ over $[\underline{h}, \bar{h}]$, the uncertain matrix $\Delta(\theta)$ is embedded in a convex polytope with $N_{v}=2^{n}$ vertices

$$
\begin{gathered}
\Delta(\theta) \in \operatorname{conv}\left\{D_{1}, D_{2}, \ldots, D_{N_{v}}\right\} \\
:=\operatorname{conv}\left\{T^{-1} \operatorname{diag}\left(\rho_{1}, \ldots, \rho_{n}\right) T: \rho_{i} \in\left\{\rho_{i}^{\text {min }}, \rho_{i}^{\max }\right\},\right. \\
i=1, \ldots, n\} .
\end{gathered}
$$

Using (77), the polytopic set (73) can be constructed with $\Lambda_{i}=I+D_{i}(A+B K), i \in \mathcal{I}$. A similar embedding procedure can be applied in the general case (when the eigenvalues of $A$ have multiplicity different than one or when they are complex) - see [43].

As the numerical complexity of the obtained LMI conditions depends significantly on the number of vertices $N_{v}$ of the polytopic approximation, one of the challenges is to provide accurate convex polytopes while reducing the number of vertices. For the Jordan decomposition procedure, the number of vertices $N_{v}$ increases exponentially with the order of the system. A method for reducing the number of vertices has been provided in $[201,157,156]$. However, the method provides a larger polytopic embedding and may result in a conservative stability condition. Methods that are independent of the order of the systems have been proposed by combining polytopic embeddings with norm bounded approximations $[108,109,53,52]$. We present briefly an adaptation of the approach based on Taylor series approximation in $[108,109]$, originally used for sampled-data systems with 
input delay. Note that the transition matrix $\Lambda\left(h_{k}\right)$ with $h_{k} \in[\underline{h}, \bar{h}]$ can be rewritten as

$$
\Lambda\left(h_{k}\right)=\Lambda(\underline{h})+\Delta\left(\rho_{k}\right) \Psi(\underline{h})
$$

where $\rho_{k}=h_{k}-\underline{h} \in[0, \bar{h}-\underline{h}], \Delta(\rho)=\int_{0}^{\rho} e^{A s} d s$ and $\Psi(\underline{h})=A \Lambda(\underline{h})+B K$. Using a Taylor series approximation of the matrix exponential, $\Delta(\rho)$ can be expressed as

$$
\Delta(\rho)=T_{M}(\rho)+R_{M}(\rho)
$$

where $T_{M}(\rho)=\sum_{i=1}^{M} \frac{A^{i-1} \rho^{i}}{i !}$ is the $M^{t h}$ order Taylor series approximation and $R_{M}(\rho)$ is the remainder. The procedure proposed in $[108,109]$ allows to embed $T_{M}(\rho)$ in a convex polytope with $N_{v}=M+1$ vertices

$$
T_{M}(\rho) \in \operatorname{conv}\left\{U_{i}, i=1, \ldots, M+1\right\}, \forall \rho \in[0, \bar{h}-\underline{h}],
$$

where $U_{0}=0, U_{i+1}=\frac{(\bar{h}-\underline{h})^{i} A^{i-1}}{i !}+U_{i}, i=1, \ldots, M$. Furthermore, an upper bound on the induced Euclidean norm of $R_{M}(\rho)$ can be computed using the method proposed in [149]. To obtain an embedding with $\left\|R_{M}(\rho)\right\|_{2}<\gamma_{R}$ for all $\rho \in[0, \bar{h}-\underline{h}]$ the approximation order $M$ must be chosen such that

$$
\frac{\|A\|_{2}(\bar{h}-\underline{h})}{M+2}<1
$$

and

$$
\frac{\left\|A^{M}\right\|_{2}(\bar{h}-\underline{h})^{M+1}}{(M+1) !} \frac{M+2}{M+2-\|A\|_{2}(\bar{h}-\underline{h})} \leq \gamma_{R} .
$$

For this approach the number of vertices is linear in the order $M$ of the Taylor approximation. Stability criteria are obtained in a direct manner by combining LMI methods for polytopic systems with the ones for systems with norm-bounded uncertainty.

Note that for both norm-bounded and polytopic embeddings approaches, the accuracy of the approximation may be significantly increased by dividing $[\underline{h}, \bar{h}]$ into several subintervals and applying the embedding procedure locally $[79,200,111,52]$. For example, in the case of the norm-bounded embedding used in Theorem 19, the idea is to consider a grid of $r$ "nominal" sampling intervals $\left\{T_{1}<T_{2}<\cdots<T_{r}\right\}$ and to verify the existence of a symmetric positive definite matrix $X$ and of $r$ parameters $\gamma_{i}, i=1, \ldots, r$, such that $\mathcal{M}\left(T_{i}, X, \gamma_{i}\right) \prec 0$ for all $i=1, \ldots, r$. When this condition is satisfied, system (62) is stable for any time-varying sampling interval $h_{k} \in$ $\cup_{i=1}^{r} \mathcal{T}\left(T_{i}, \gamma_{i}\right)$ where $\mathcal{T}\left(T_{i}, \gamma_{i}\right)=\left[\underline{h}\left(T_{i}, \gamma_{i}\right), \bar{h}\left(T_{i}, \gamma_{i}\right)\right]$ are defined using (71), (72). Furthermore, it has been shown in [79] that using this approach one can approximate the condition (65) as accurately as desired, in the sense that if the condition (65) holds for $\theta \in[\underline{h}, \bar{h}]$, then necessarily there exists a matrix $X=P^{-1}$, a sufficiently tight grid of parameters $T_{i}, i=1, \ldots, r$ and positive scalars $\gamma_{i}, i=1, \ldots, r$, such that $\mathcal{M}\left(T_{i}, X, \gamma_{i}\right) \prec 0$ for all $i=1, \ldots, r$, and $[\underline{h}, \bar{h}] \subset \cup_{i=1}^{r} \mathcal{T}\left(T_{i}, \gamma_{i}\right)$. Such an asymptotic exactness property has also been discussed for other embedding approaches $[52,200,241]$. The main issue is that, using convex embeddings, the conservatism with respect to the quadratic stability condition (65) can be reduced to any degree at the cost of increased computational complexity. However, the analysis of the asymptotic exactness property does not take into account all numerical implementation aspects. Most of the methods are based on the computation of the matrix exponential for nominal sampling intervals, on the use of the eigenvalues/eigenvectors of the state matrix $A$ or of its characteristic polynomial, etc. Computing any of these elements introduces approximations [179] which might influence the numerical implementation of the embedding. The effect of these approximations on the accuracy of the stability analysis needs to be further analysed.

\subsubsection{Analysis based on Delta-operators}

One of the drawbacks of the discrete-time analysis as the one proposed in (65) is the fact that the matrix $\Lambda(\theta)$ is close to identity when $\theta$ is small. For small values of the lower bound of the sampling interval $\underline{h}$, the inequality may be difficult to handle numerically. To avoid this numerical drawback, a condition which encompasses (65) has been proposed in [200].

Theorem 20 System (12) is Exponentially Stable for any arbitrary sampling sequence with $t_{k+1}-t_{k} \in[\underline{h}, \bar{h}]$ if there exists $X \succ 0$ such that

$$
\Omega(\theta) X+X \Omega^{T}(\theta)+\theta \Omega^{T}(\theta) X \Omega(\theta) \prec 0,
$$

for all $\theta \in[\underline{h}, \bar{h}]$ where

$$
\Omega(\theta)=\frac{1}{\theta}(\Lambda(\theta)-I)=\frac{1}{\theta} \int_{0}^{\theta} e^{A s} d s(A+B K) .
$$

This condition is obtained from an extension of the Delta-operator approach [171]. System (12) with $h_{k} \in[\underline{h}, \bar{h}]$ satisfies the relation

$$
\delta_{k} x=\Omega\left(h_{k}\right) x\left(t_{k}\right), \forall k \in \mathbb{N},
$$

where $\delta_{k} x:=\frac{x\left(t_{k+1}\right)-x\left(t_{k}\right)}{h_{k}}, \forall k \in \mathbb{N}$, and $\Omega(\cdot)$ as defined in (81). Note that when $h$ tends to zero, the matrix $\Omega(h)$ converges to $A+B K$. The model (82) provides a smooth transition from the continuous-time control loop $\dot{x}=(A+B K) x$ (obtained when the sampling interval tends to zero) and the discrete-time representation (62). Condition (80) implies that the candidate 
Lyapunov function $V(x)=x^{T} X^{-1} x$ satisfies

$$
\delta_{k} V:=\frac{V\left(x_{k+1}\right)-V\left(x_{k}\right)}{h_{k}}<0
$$

for all $x_{k} \neq 0, h_{k} \in[\underline{h}, \bar{h}], k \in \mathbb{N}$. When $\theta$ tends to zero, (80) converges to the classical stability conditions for the continuous-time control loop:

$$
(A+B K) X+X(A+B K)^{T} \prec 0 .
$$

Similarly to the discrete-time analysis in condition (65), the parametric conditions (80) can be replaced by a finite number of LMIs using a convex embedding of the matrix $\Omega(\theta)$. See [200], where a polytopic embedding procedure based on the Jordan normal form and the mean value theorem has been proposed. See also [29] for an approach using more general Lyapunov functions.

\subsubsection{Continuous-time analysis using embeddings}

In practice it is important to provide an estimate of the system's performance in between sampling instants. A continuous-time approach based on convexification arguments has been proposed in $[114,61]$ for LTI systems. The approach takes into account the relation

$$
x(t)=\Lambda\left(t-t_{k}\right) x\left(t_{k}\right), \forall t \in\left[t_{k}, t_{k+1}\right), k \in \mathbb{N},
$$

still referring to the definition of the transition matrix $\Lambda\left(t-t_{k}\right)=I+\int_{0}^{t-t_{k}} e^{A s} d s(A+B K)$ of system (12). The classical condition $\dot{V}(x(t))<-\lambda V(x(t))$ ensuring the exponential decay of a candidate Lyapunov function $V(x)=x^{T} P x$ for some positive $\lambda$ can be expressed as the following parametric LMI

$$
\left[\begin{array}{c}
\Lambda(\theta) \\
I
\end{array}\right]^{T}\left[\begin{array}{cc}
A^{T} P+P A+\lambda P & P B K \\
K^{T} B^{T} P & 0
\end{array}\right]\left[\begin{array}{c}
\Lambda(\theta) \\
I
\end{array}\right] \prec 0,
$$

for all $\theta \in[0, \bar{h}]$. A finite number of LMIs can be obtained similarly to the discrete-time case, by embedding the matrix $\Lambda(\theta)$ in a convex polytope

$$
\tilde{\mathcal{W}}:=\operatorname{conv}\left\{\tilde{\Lambda}_{1}, \tilde{\Lambda}_{2}, \cdots, \tilde{\Lambda}_{N_{v}}\right\},
$$

that is $\Lambda(\theta) \in \tilde{\mathcal{W}}, \forall \theta \in[0, \bar{h}]$. Sufficient stability conditions [114] are given by the existence of a matrix $P \succ 0$ and of matrices $G_{1}, G_{2} \in \mathbb{R}^{n \times n}$ solution to

$$
\left[\begin{array}{cc}
A^{T} P+P A+\lambda P+G_{1}+G_{1}^{T} & P B K-G_{1} \tilde{\Lambda}_{i}+G_{2}^{T} \\
K^{T} B^{T} P-\tilde{\Lambda}_{i}^{T} G_{1}^{T}+G_{2} & -G_{2} \tilde{\Lambda}_{i}-\tilde{\Lambda}_{i}^{T} G_{2}^{T}
\end{array}\right] \prec 0
$$

for all $i=1, \ldots, N_{v}$. For a less conservative approach using the Lyapunov-Razumikhin method, see [61]. A robust analysis faced to perturbations has been proposed in [63].

\subsubsection{Looped functionals}

While convex embeddings allow for approximating the stability conditions (65) as accurately as desired, including parametric uncertainties in the analysis is quite difficult. Recently, a discrete-time approach based on the so-called looped-functionals has been considered in [232] to deal with this issue. The key idea is to apply the functional framework, as in the time-delay approach, while preserving the accuracy of discrete-time stability conditions. The approach relies on an unusual formulation of sampled-data systems inspired from the lifting modelling [269]. For all $k \in \mathbb{N}$, consider a lifted state function $\chi_{k}(\cdot)$ satisfying

$$
\left\{\begin{array}{l}
\chi_{k+1}(0)=\chi_{k}\left(h_{k}\right), \forall k \in \mathbb{N}, h_{k} \in[\underline{h}, \bar{h}], \\
\frac{\mathrm{d}}{\mathrm{d} \tau} \chi_{k}(\tau)=A \chi_{k}(\tau)+B K \chi_{k}(0), \forall \tau \in\left[0, h_{k}\right] .
\end{array}\right.
$$

The function $\chi_{k}(\tau), \tau \in\left[0, h_{k}\right]$ represents the trajectory of the sampled-data system over the interval $\left[t_{k}, t_{k+1}\right]$. Define the set $\mathbb{K}=\bigcup_{h \in[h, \bar{h}]} \mathcal{C}^{0}\left([0, h], \mathbb{R}^{n}\right)$ so to represent such a class of functions. The following theorem establishes a relation between a discrete-time stability analysis (as in (66)) and an analysis using functionals (as in the time-delay approach).

Theorem 21 [232] Consider system (12) and the lifted model (87). Let $V: \mathbb{R}^{n} \rightarrow \mathbb{R}_{+}$be a differentiable function for which there exist positive scalars $\mu_{1}<\mu_{2}$ such that for all $x \in \mathbb{R}^{n}, \mu_{1}\|x\|^{2} \leq V(x) \leq \mu_{2}\|x\|^{2}$. The two following statements are equivalent.

(i) The increment of the candidate Lyapunov function $V(\cdot)$ is strictly negative at sampling instants, i.e.

$$
\Delta V(k)=V\left(\chi_{k}\left(h_{k}\right)\right)-V\left(\chi_{k}(0)\right)<0,
$$

for all $k \in \mathbb{N}$ and $h_{k} \in[\underline{h}, \bar{h}]$;

(ii) There exists a continuous and differentiable functional $\mathcal{V}:[0, \bar{h}] \times \mathbb{K} \rightarrow \mathbb{R}$ which satisfies for all $h \in$ $[\underline{h}, \bar{h}], z \in \mathcal{C}^{0}\left([0, h], \mathbb{R}^{n}\right) \subset \mathbb{K}$

$$
\mathcal{V}(h, z(\cdot))=\mathcal{V}(0, z(\cdot)),
$$

and such that, for all $\left(k, h_{k}, \tau\right) \in \mathbb{N} \times[\underline{h}, \bar{h}] \times\left[0, h_{k}\right]$,

$$
\dot{\mathcal{W}}\left(\tau, \chi_{k}\right)=\frac{\mathrm{d}}{\mathrm{d} \tau}\left[V\left(\chi_{k}(\tau)\right)+\mathcal{V}\left(\tau, \chi_{k}\right)\right]<0 .
$$

Moreover, if one of these two statements is satisfied, then the null solution of system (12) is Asymptotically Stable for any sampling sequence with $t_{k+1}-t_{k} \in[\underline{h}, \bar{h}]$.

The main difference compared with the LyapunovKrasovkii approach used in the time-delay framework remains in the design of the functional. The positive 
definiteness condition of the LKF is exchanged with a looping-condition, a two-point algebraic equality (88) that the functional should verify; see e.g. [232,3032,237 . There are two main methods for building loop functionals. The first manner follows the construction of discontinuous Lyapunov-functionals, as for instance (48), (50) or the ones provided in $[67,187,230,154]$. The previous theorem first states that if a term of the functional meets the looping condition (88), then the positive definiteness of this term can be relaxed. The second method enters into the framework of polynomials functions $[31,30,32,237]$ and of sum of squares tools [222]. For example, in [237], a polynomial looped-functional was introduced and it has the following form

$$
\mathcal{V}\left(\tau, \chi_{k}\right)=\left[\begin{array}{l}
\chi_{k}(\tau) \\
\chi_{k}(0)
\end{array}\right]^{T} M\left(\tau, h_{k}\right)\left[\begin{array}{l}
\chi_{k}(\tau) \\
\chi_{k}(0)
\end{array}\right]
$$

where $M$ is a polynomial matrix function from $[0, \bar{h}] \times$ $[\underline{h}, \bar{h}]$ to the set of $2 n \times 2 n$ symmetric matrices. The looping condition (88) is ensured by adding the following constraints on $M$ :

$\forall h \in[\underline{h}, \bar{h}], \quad\left[\begin{array}{l}I \\ I\end{array}\right]^{T} M(0, h)\left[\begin{array}{l}I \\ I\end{array}\right]=0$, and $M(h, h)=0$.

This method can easily deal with polytopic uncertainties in the system matrices.

\subsubsection{A discrete-time approach for nonlinear systems}

Results on discrete-time approaches for the control of nonlinear systems with time-varying sampling intervals are quite rare. We present as follows an adaptation of the result from [258] which extends earlier stability criteria from $[191,196,190]$. Consider the nonlinear system

$$
\dot{x}(t)=F(x(t), u(t))
$$

with $F(x, u)$ globally Lipschitz, i.e. there exists $\beta_{f}>0$ such that

$$
\left\|F\left(x_{a}, u_{a}\right)-F\left(x_{b}, u_{b}\right)\right\| \leq \beta_{f}\left(\left\|x_{a}-x_{b}\right\|+\left\|u_{a}-u_{b}\right\|\right)
$$

for all $x_{a}, x_{b} \in \mathbb{R}^{n}$ and $u_{a}, u_{b} \in \mathbb{R}^{m}$. The control takes the form $u(t)=u_{k}$ for all $t \in\left[t_{k}, t_{k+1}\right)$ and the sampling interval is bounded $h_{k}=t_{k+1}-t_{k} \in \mathcal{T}=[\underline{h}, \bar{h}], \forall k \in \mathbb{N}$. The exact discrete-time model of the system over the sampling interval is given by

$$
x_{k+1}=F_{h_{k}}^{e}\left(x_{k}, u_{k}\right):=x_{k}+\int_{t_{k}}^{t_{k}+h_{k}} F\left(x(s), u_{k}\right) d s
$$

where $x_{k}=x\left(t_{k}\right)$. Note however that (91) is not known in general since it is rare to obtain an analytic solution to a nonlinear initial value problem. In practical problems, approximations are usually used [244,191]. A simple example is given by the Euler model of (90):

$$
x_{k+1}=x_{k}+h_{k} F\left(x_{k}, u_{k}\right) \text {. }
$$

Other approximations can be found in standard books [244] and tutorials [182,183]. The approach in [258] considers an approximate model

$$
x_{k+1}=F_{h^{*}}^{a}\left(x_{k}, u_{k}\right) \text {, }
$$

obtained for some nominal sampling interval $h^{*} \in[\underline{h}, \bar{h}]$. Model (92) is assumed to be one-step consistent [244] with the exact discrete-time plant, i.e. there exists $\hat{\rho} \in \mathcal{K}_{\infty}$ such that $\left\|F_{h^{*}}^{a}(x, u)-F_{h^{*}}^{e}(x, u)\right\| \leq$ $h^{*} \hat{\rho}\left(h^{*}\right)(\|x\|+\|u\|)$, for all $x \in \mathbb{R}^{n}, u \in \mathbb{R}^{m}$. It is considered that the approximate model (92) has been used to design a controller

$$
u_{k}=K_{h^{*}}\left(x_{k}\right)
$$

parametrized by the nominal sampling interval $h^{*}$, and that the closed-loop system $(92),(93)$ is asymptotically stable. More formally, it is assumed that there exists a candidate Lyapunov function for the approximate closed-loop system $(92),(93)$, i.e. a function $V_{h^{*}}(x)$ and $\alpha_{i}>0, i=1,2,3$ such that the involved conditions holds for some $r>1: \alpha_{1}\|x\|^{r} \leq V_{h^{*}}(x) \leq \alpha_{2}\|x\|^{r}$ and

$$
\frac{V_{h^{*}}\left(F_{h^{*}}^{a}\left(x, K_{h^{*}}\left(x_{k}\right)\right)\right)-V_{h^{*}}(x)}{h} \leq-\alpha_{3}\|x\|^{r}
$$

for all $x \in \mathbb{R}^{n}$. Furthermore, the control law $K_{h^{*}}(\cdot)$ is considered to be linearly bounded, i.e. there exists $\beta_{u}>$ 0 such that $\left\|K_{h^{*}}(x)\right\| \leq \beta_{u}\|x\|$ for all $x \in \mathbb{R}^{n}$. The following theorem provides generic results for the robust stability of the exact closed-loop system

$$
x_{k+1}=F_{h_{k}}^{e}\left(x_{k}, K_{h^{*}}\left(x_{k}\right)\right) \text {, }
$$

using the fact that the control law $u_{k}=K_{h^{*}}\left(x_{k}\right)$ is a stabilizer for the approximate model (92).

Theorem 22 [258] Consider system (95) with $h_{k} \in$ $[\underline{h}, \bar{h}]$ for all $k \in \mathbb{N}$. Consider the following notation

$$
\begin{gathered}
\beta_{a}=\left(2+\beta_{u}+\left(1+\max \left(1, \beta_{u}\right)\right)\left(e^{\beta_{f} \bar{h}}-1\right)\right) \\
+h^{*} \hat{\rho}\left(h^{*}\right)\left(1+\beta_{u}\right) .
\end{gathered}
$$

Assume that the Lyapunov candidate function $V_{h^{*}}(x)$ is locally Lipschitz and there exists $\beta_{v}>0$ such that

$$
\sup _{z \in V_{h^{*}}(x)}\|z\| \leq \beta_{v}\|x\|^{r}
$$


for all $x \in \mathbb{R}^{n}$, where $\partial V_{h^{*}}(x)$ denotes the generalized differential of Clarke. If there exists $\beta \in(0,1)$ such that

$$
\frac{\beta_{v} \beta_{a}^{r-1}}{h^{*}}\left(h^{*} \hat{\rho}\left(h^{*}\right)\left(1+\beta_{u}\right)+\rho_{h}\left(h^{*}, M_{h}\right)\right) \leq(1-\beta) \alpha_{3}
$$

is satisfied where

$$
\rho_{h}\left(h^{*}, M_{h}\right)=e^{\beta_{f} h^{*}}\left(\left(1+\beta_{u}\right)\left(e^{\beta_{f} M_{h}}-1\right)\right)
$$

with $M_{h}=\max _{h \in[\underline{h}, \bar{h}]}\left|h-h^{*}\right|$, then there exist $c, \lambda>0$ such that $\left\|x_{k}\right\| \leq c\left\|x_{0}\right\| e^{-\lambda k \underline{h}}$. In other words, system (95) is Globally Exponentially Stable, Uniformly for all $h_{k} \in[\underline{h}, \bar{h}]$ and all $k \in \mathbb{N}$.

The above theorem is a natural extension of the result in $[190,191]$ for sampled-data systems with constant sampling intervals. The main condition (97) involves two terms. The first term $\beta_{v} \beta_{a}^{r-1} \hat{\rho}\left(h^{*}\right)\left(1+\beta_{u}\right)$ reflects the effect of approximatively discretizing the nominal system using a nominal sampling interval $h^{*}$; the second one, $\frac{\beta_{v} \beta_{a}^{r-1}}{h^{*}} \rho_{h}\left(h^{*}, M_{h}\right)$ reflects the effect of uncertainty in the sampling interval.

\subsubsection{Further reading}

Control design methodologies based on convex embeddings have been presented in $[108,43,109,83,185]$. See also [226] for an LPV design of controllers that are adapted in real time to the value of the sampling interval and [110] for the case of systems with delay scheduled controllers. Extensions of the discrete-time approach for networked control systems with scheduling protocols can be found in $[53,146,52,147,36]$. For model predictive control of networked control systems see also $[201,89,157]$. Lie algebraic criteria for the analysis of systems with time varying sampling have been proposed in [58]. A mixed continuous-discrete approach has also been proposed in [148]. The relation between clock dependent and looped Lyapunov functionals has been investigated in [28].

\subsection{Input/Output stability approach}

In this subsection we present several methods that study sampled-data systems from a robust control point of view. The main idea of the Input/Output stability approach is to consider the sampling error as a perturbation with respect to a nominal continuous-time control-loop. Classical robust control tools are used in order to assess the stability of the sampled-data systems $[272,278,168,217]$. Some of the presented methods are reminiscent from the Input/Output stability approach used for the analysis of time delay systems $[122,124,197,96,130,77,131]$, and have been further developed independently of the time delay approach.

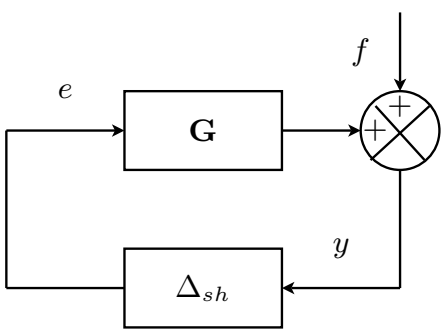

Fig. 6. Equivalent representation of the sampled-data system, from a robust control theory point of view.

\subsubsection{Basic idea}

Note that the LTI sampled-data system (12) can be reexpressed in the form [174]

$$
\dot{x}(t)=(\underbrace{A+B K}_{:=A_{c l}}) x(t)+\underbrace{B K}_{:=B_{c l}}(\underbrace{x\left(t_{k}\right)-x(t)}_{:=e(t)}),
$$

where $A_{c l}$ corresponds to the state matrix of the nominal continuous-time control loop while $e(t)$ represents the error induced by sampling. An essential fact in this approach is that the sampling induced error $e(t)=x\left(t_{k}\right)-$ $x(t)$ can be equivalently re-expressed as

$$
e(t)=-\int_{t_{k}}^{t} \dot{x}(\theta) d \theta, \forall t \in\left[t_{k}, t_{k+1}\right) .
$$

Considering $y(t)=\dot{x}(t)$ as an auxiliary output for system (98), the sampled-data system (12) can be represented equivalently by the feedback interconnection of the operator $\Delta_{s h}: \mathcal{L}_{2 e}^{n}[0, \infty) \rightarrow \mathcal{L}_{2 e}^{n}[0, \infty), \Delta_{s h}: y \rightarrow e$, defined by:

$$
e(t)=\left(\Delta_{s h} y\right)(t)=-\int_{t_{k}}^{t} y(\theta) d \theta, \quad \forall t \in\left[t_{k}, t_{k+1}\right),
$$

with the system

$$
\left\{\begin{array}{l}
\dot{x}(t)=A_{c l} x(t)+B_{c l} e(t), x(0)=x_{0} \in \mathbb{R}^{n} \\
y(t)=C_{c l} x(t)+D_{c l} e(t)=\dot{x}(t)
\end{array}\right.
$$

where $C_{c l}=A_{c l}=A+B K$ and $D_{c l}=B_{c l}=B K$. Note that the nominal system (101) is LTI. It represents the dynamics of the continuous-time system with an additive input perturbation $e$. The operator $\Delta_{s h}$ captures both the effects of sampling and its variations. An alternative model can also be derived by considering the actuation error $e_{u}(t)=K\left(x\left(t_{k}\right)-x(t)\right)$ (see [81]). The stability of the sampled-data system (12) can then be studied by analysing the interconnection (100),(101).

\subsubsection{Small gain conditions}

To provide constructive stability conditions, the Small Gain Theorem [272,278,122,96] constitutes a simple and 
powerful tool in the robust control framework. Let $\mathbf{G}$ : $\mathcal{L}_{2}^{n}[0, \infty) \rightarrow \mathcal{L}_{2}^{n}[0, \infty)$ be the linear operator described by the transfer function

$$
\hat{\mathbf{G}}(s)=s\left(s I-A_{c l}\right)^{-1} B_{c l}
$$

associated to system (101). The operator G captures the behaviour of (101) for null initial conditions. Considering the free response of system (101), $f(t)=A_{c l} e^{A_{c l} t} x_{0}, \quad \forall t \geq 0$, the interconnection (100),(101) can be re-expressed as

$$
\left\{\begin{array}{l}
y=\mathbf{G} e+f \\
e=\Delta_{s h} y
\end{array}\right.
$$

(see Figure 6). A direct consequence of the Small Gain Theorem is the fact that if

$$
\|\mathbf{G}\|_{2,2}\left\|\Delta_{s h}\right\|_{2,2}<1
$$

then the interconnection (103) is $\mathcal{L}_{2}$ stable, i.e. there exist a positive scalar $C$ such that

$$
\int_{0}^{t}\left(\|y(\theta)\|^{2}+\|e(\theta)\|^{2}\right) d \theta \leq C \int_{0}^{t}\|f(\theta)\|^{2} d \theta
$$

for any $t>0$. Here $\|\mathbf{G}\|_{2,2},\left\|\Delta_{s h}\right\|_{2,2}$ denote the induced $\mathcal{L}_{2}$ norms of $\mathbf{G}$ and $\Delta_{s h}$, respectively ${ }^{4}$. Inequality (104) is known as the small gain condition. Due to the linearity of $\mathbf{G}$, its induced $\mathcal{L}_{2}$ norm can be readily computed [278] using the $\mathcal{H}_{\infty}$ norm of its transfer function:

$$
\|\mathbf{G}\|_{2,2}=\|\mathbf{G}\|_{\infty}:=\sup _{\omega \in \mathbb{R}} \bar{\sigma}(\hat{\mathbf{G}}(j \omega)) .
$$

Furthermore, for the case of LTI sampled-data systems, $\mathcal{L}_{2}$ stability of the interconnection (103) implies asymptotic stability ${ }^{5}$ of the sampled-data control loop (12):

Theorem 23 [81] Suppose that $A_{c l}$ is Hurwitz. System (12) is Uniformly Asymptotically Stable if the feedback interconnection (103) is $\mathcal{L}_{2}$ stable.

Therefore, providing tractable stability conditions for system (12) leads to providing an estimate for the induced $\mathcal{L}_{2}$ norm of the operator $\Delta_{s h}$. An upper bound of this norm has been computed in [130] using a more general uncertain delay operator:

$$
\Delta_{d}: y(t) \rightarrow e(t)=\left(\Delta_{d} y\right)(t):=-\int_{t-\tau(t)}^{t} y(\theta) d \theta
$$

\footnotetext{
$\overline{{ }^{4} \text { Given }}$ an operator $\mathbf{G}: \mathcal{L}_{2}^{n}[0, \infty) \rightarrow \mathcal{L}_{2}^{n}[0, \infty)$, its induced $\mathcal{L}_{2}$ norm is defined as $\|\mathbf{G}\|_{2,2}:=\sup _{u \neq 0} \frac{\|\mathbf{G} u\|_{\mathcal{L}_{2}}}{\|u\|_{\mathcal{L}_{2}}}$.

${ }^{5}$ For relations with exponential stability see also [68].
}

where $\tau(t) \in[0, \bar{h}]$. The operator $\Delta_{s h}$ is a particular case of $\Delta_{d}$ with $\tau(t)=t-t_{k}, \forall t \geq 0, k \in \mathbb{N}$.

Lemma 24 [130] The $\mathcal{L}_{2}$-induced norm of the operator $\Delta_{d}$ in (106) is bounded by $\bar{h}$.

Using this property, and the fact that the operator $\Delta_{d}$ satisfies $M \Delta_{d}=\Delta_{d} M$ for all $M \in \mathbb{R}^{n \times n}$, Mirkin [174] provided the following $\mathcal{L}_{2}$ stability conditions

$\exists M \in \mathbb{R}^{n \times n}, M \succ 0 \quad$ such that $\left\|M \hat{\mathbf{G}}(s) M^{-1}\right\|_{\infty}<\frac{1}{\bar{h}}$,

which is a consequence of the Scaled Small Gain Theorem [242]. Interestingly, it is also shown that (107) is related to the condition in [72] which is obtained using the input-delay approach and the Lyapunov-Krasovskii functional (23). The same LMI can be used to check both conditions. Mirkin then showed that the bound on the $\mathcal{L}_{2}$ induced norm can be enhanced by exploiting the properties of $\Delta_{s h}$.

Lemma 25 [174] The $\mathcal{L}_{2}$-induced norm of the operator $\Delta_{\text {sh }}$ is bounded by $\delta_{0}=\frac{2}{\pi} \bar{h}$, and thus

$$
\int_{0}^{+\infty}\left\|\left(\Delta_{s h} y\right)(\theta)\right\|^{2} d \theta \leq \int_{0}^{+\infty} \delta_{0}^{2}\|y(\theta)\|^{2} d \theta
$$

for all $y \in \mathcal{L}_{2}^{n}[0, \infty)$.

This bound on the induced $\mathcal{L}_{2}$ norm of $\Delta_{s h}$ is actually exact and it is attained when there exists an index $k \in \mathbb{N}$ such that $t_{k+1}-t_{k}=\bar{h}$. This leads to the following sufficient $\mathcal{L}_{2}$ stability condition, improving (107):

$\exists M \in \mathbb{R}^{n \times n}, M \succ 0 \quad$ such that $\left\|M \hat{\mathbf{G}}(s) M^{-1}\right\|_{\infty}<\frac{\pi}{2 \bar{h}}$.

Note that the upper bound on induced $\mathcal{L}_{2}$ norm of $\Delta_{s h}$ can also be related to the Wirtinger's inequalities [154] used in the time delay approach. In practice, condition (109) is readily verifiable via standard LMI for the estimation of the $\mathcal{H}_{\infty}$ norm of LTI systems $[174,242,96]$

$$
\left[\begin{array}{ccc}
X A_{c l}+A_{c l}^{T} X & \frac{2}{\pi} \bar{h} X B K & A_{c l}^{T} Y \\
* & -Y & \frac{2}{\pi} \bar{h} K^{T} B^{T} Y \\
* & * & -Y
\end{array}\right] \prec 0
$$

to be solved for $X, Y \succ 0$ (obtained with $Y=M^{2}$ ).

Recently, an extension of the Input-Output stability approach has been proposed [40] for nonlinear systems

$$
\left\{\begin{array}{l}
\dot{x}=f(x)+g(x) u \\
y=H(x)
\end{array}\right.
$$


with a sampled-data output feedback

$$
u(t)=K\left(y\left(t_{k}\right)\right), \forall t \in\left[t_{k}, t_{k+1}\right) .
$$

Here $f(0)=0$ and all the functions are supposed to be continuously differentiable. The closed-loop system (111), (112) can be re-expressed as

$$
\begin{aligned}
\dot{x}(t)= & (\underbrace{f(x)+g(x) K(H(x))}_{:=f_{c l}(x)}) \\
& -g(x)(\underbrace{K(y(t))-K\left(y\left(t_{k}\right)\right)}_{:=e_{u}(t)}),
\end{aligned}
$$

where $f_{c l}(x)$ represents the dynamics of a nominal closed-loop system and $e_{u}$ the error of sampling at controller level. This model can be related with (60) used in the impulsive approach by [193]. The sampling error satisfies

$$
e_{u}(t)=\int_{t_{k}}^{t} z(\theta) d \theta, \forall t \in\left[t_{k}, t_{k+1}\right)
$$

where $z(t)=d K(y(t)) / d t$. Stability conditions can be derived based on Input-to-State stability and small gain analysis.

Theorem 26 [40] Consider system (111) under the control law (112). Assume that $t_{k+1}-t_{k} \in[0, \bar{h}]$. Suppose that the following auxiliary system

$$
\left\{\begin{array}{l}
\dot{x}(t)=f_{c l}(x(t))-g(x(t)) e_{u}(t) \\
z(t)=\frac{d K(H(x(t)))}{d t}
\end{array}\right.
$$

with input $e_{u}$ and output $z$ has the following Input-toState and Input-to-Output Stability properties

$$
\begin{aligned}
\|x(t)\| & \leq \max \left\{\beta_{x}\left(\left\|x\left(\tau_{0}\right)\right\|, t-\tau_{0}\right), \gamma_{x}\left(\left\|e_{u\left[\tau_{0}, t\right]}\right\|\right)\right\},(11 \\
\|z(t)\| & \leq \max \left\{\beta\left(\left\|x\left(\tau_{0}\right)\right\|, t-\tau_{0}\right), \gamma\left(\left\|e_{u\left[\tau_{0}, t\right]}\right\|\right)\right\}
\end{aligned}
$$

$\forall t \geq \tau_{0} \geq 0$, where $\left\|e_{u\left[\tau_{0}, t\right]}\right\|:=\sup _{t \in\left[\tau_{0}, t\right]}\left\|e_{u}(t)\right\|, \beta, \beta_{x}$ are class $\mathcal{K} \mathcal{L}$ functions and $\gamma, \gamma_{x}$ are class $\mathcal{K}$ functions. If $\gamma(s)<s / \bar{h}$ for all $s>0$, then the equilibrium point $x=0$ of the closed-loop system (111), (112) is Globally Asymptotically Stable for any initial condition in $\mathbb{R}^{n}$.

\subsubsection{Integral Quadratic Constraints and extensions}

For the case of LTI sampled-data systems (12), the properties of the operator $\Delta_{s h}$ in $(100)$ can be further exploited in the framework of Integral Quadratic Constraints (IQC) [168]. Less conservative stability conditions can be obtained. While very general definitions of IQCs are available in the literature [168], we restrict ourselves here to IQCs defined by symmetric matrices
$\Pi$ with real elements, that have been used for stability analysis of systems with aperiodic sampling. Roughly speaking, the bounded operator $\Delta_{s h}$ in (100), with input $y$ and output $e$, is said to satisfy the IQC defined by the symmetric matrix $\Pi$ if

$$
\int_{0}^{\infty}\left[\begin{array}{l}
y(\theta) \\
e(\theta)
\end{array}\right]^{T} \Pi\left[\begin{array}{l}
y(\theta) \\
e(\theta)
\end{array}\right] d \theta \geq 0
$$

for all $y \in \mathcal{L}_{2}^{n}[0, \infty)$ and $e=\Delta_{s h} y$. We present as follows a simplified version of the classical IQC Theorem [168] that can be used in order to derive stability conditions for the interconnection (103).

Theorem 27 [168] Consider the interconnection (103) describing the LTI sampled-data system (12) and the bounded operator $\Delta_{s h}$ in (100). Suppose that $A_{c l}=A+$ $B K$ is Hurwitz and assume that there exists a matrix

$$
\Pi=\left[\begin{array}{ll}
\Pi_{11} & \Pi_{12} \\
\Pi_{12}^{T} & \Pi_{22}
\end{array}\right]
$$

with $\Pi_{11}, \Pi_{12}, \Pi_{22} \in \mathbb{R}^{n \times n}, \Pi_{11} \succeq 0, \Pi_{22} \preceq 0$, such that the operator $\Delta_{\text {sh }}$ satisfies the $I Q C$ defined by $\Pi$; there exists $\epsilon>0$ such that

$$
\left[\begin{array}{c}
\hat{\boldsymbol{G}}(j \omega) \\
I
\end{array}\right]^{\star} \Pi\left[\begin{array}{c}
\hat{\boldsymbol{G}}(j \omega) \\
I
\end{array}\right] \preceq-\epsilon I, \forall \omega \in \mathbb{R} .
$$

Then the interconnection (103) is $\mathcal{L}_{2}$ stable.

Using Theorem 23, the conditions of Theorem 27 also imply uniform asymptotic stability of the sampled-data system (12). Condition (120) can be converted into a frequency independent finite dimensional LMI using the Kalman-Yakubovich-Popov Lemma [223]:

$$
\left[\begin{array}{cc}
A_{c l}^{T} P+P A_{c l} & P B_{c l} \\
B_{c l}^{T} P & 0
\end{array}\right]+\left[\begin{array}{cc}
C_{c l} & D_{c l} \\
0 & I
\end{array}\right]^{T} \Pi\left[\begin{array}{cc}
C_{c l} & D_{c l} \\
0 & I
\end{array}\right] \prec 0
$$

to be solved for $P \succ 0$.

As an example, a simple IQC can be obtained directly from Lemma 25. Note that inequality (108) implies that $\Delta_{s h}$ satisfies the IQC defined by

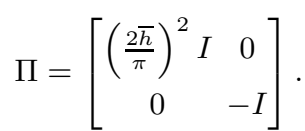

For this IQC, condition (120) yields to the standard small gain criteria

$$
\left(\frac{2 \bar{h}}{\pi}\right)^{2} \hat{\mathbf{G}}^{\star}(j \omega) \hat{\mathbf{G}}(j \omega) \prec I, \forall \omega \in \mathbb{R},
$$


which corresponds to a simple condition on the $H_{\infty}$ norm of $\mathbf{G}:\|\hat{\mathbf{G}}(s)\|_{\infty}<\frac{\pi}{2 \bar{h}}$.

Fujioka [81] showed that the operator $\Delta_{s h}$ also satisfies the following passivity-like property.

Lemma 28 [81] The operator $\Delta_{\text {sh }}$ defined in (100) satisfies

$$
\int_{0}^{+\infty} y^{T}(\theta)\left(\Delta_{s h} y\right)(\theta) d \theta \leq 0,
$$

for all $y \in \mathcal{L}_{2}^{n}[0, \infty)$.

It is important to note that if $\Delta_{s h}$ satisfies several IQC defined by matrices $\Pi_{1}, \Pi_{2}, \ldots, \Pi_{r}$, then a sufficient condition for stability that takes into account all the properties is given by the existence of positive scalars $\alpha_{1}, \alpha_{2}, \ldots, \alpha_{r}$ such that condition (120) holds with $\Pi=\alpha_{1} \Pi_{2}+\alpha_{2} \Pi_{2}+\ldots, \alpha_{r} \Pi_{r}$. The properties of $\Delta_{s h}$ in Lemma 25 and Lemma 28 can be generalized [81] using scaling matrices $0 \preceq Y \in \mathbb{R}^{n \times n}, 0 \prec X \in \mathbb{R}^{n \times n}$ and grouped into the following IQC:

$$
\int_{0}^{\infty}\left[\begin{array}{l}
y(\theta) \\
e(\theta)
\end{array}\right]^{T}\left[\begin{array}{ll}
\delta_{0}^{2} X & -Y \\
-Y & -X
\end{array}\right]\left[\begin{array}{l}
y(\theta) \\
e(\theta)
\end{array}\right] d \theta \geq 0
$$

which holds for all $y \in \mathcal{L}_{2}^{n}[0, \infty)$ and $e=\Delta_{\text {sh }} y$ with $\delta_{0}=\frac{2 \bar{h}}{\pi}$. Using the integral property (125) and Theorem 23 , Fujioka [81] has proposed the following stability condition.

Theorem 29 [81] The system (12) is Globally Uniformly Asymptotically Stable for any sampling sequence with $t_{k+1}-t_{k} \leq \bar{h}$ if there exist $0 \prec P \in \mathbb{R}^{n \times n}$, $0 \prec X \in \mathbb{R}^{n \times n}, 0 \preceq Y \in \mathbb{R}^{n \times n}$ satisfying

$$
\begin{gathered}
{\left[\begin{array}{cc}
A_{c l}^{T} P+P A_{c l} & P B_{c l} \\
B_{c l}^{T} P & 0
\end{array}\right]} \\
+\left[\begin{array}{cc}
C_{c l} & D_{c l} \\
0 & I
\end{array}\right]^{T}\left[\begin{array}{cc}
\delta_{0}^{2} X & -Y \\
-Y & -X
\end{array}\right]\left[\begin{array}{cc}
C_{c l} & D_{c l} \\
0 & I
\end{array}\right] \prec 0 .
\end{gathered}
$$

Taking into account more properties of the operator $\Delta_{s h}$ may lead to less conservative results. Nevertheless, since the analysis is of a frequency domain nature, the IQC approach is only applicable to LTI systems. However, one may note that input delays, several performance specifications and classical nonlinearities (sector bounded, saturations, etc.) can be characterized by elementary operators and IQCs [168]. A more complex system can be described by an interconnection of an LTI system and a single block diagonal operator representing the different perturbing elements. Once the IQCs for the different perturbing elements are available, stability of more complex systems is then a rather straightforward matter of defining a single aggregate IQC. This point enhances the applicability of the IQC approach.

In the nonlinear case, an extension $[206,207,203]$ of the IQC approach is possible using methods inspired by the Dissipativity Theory $[267,262]$. Consider the following nonlinear affine system:

$\dot{x}(t)=f(x(t))+g(x(t)) K\left(x\left(t_{k}\right)\right), \forall t \in\left[t_{k}, t_{k+1}\right), k \in \mathbb{N}$.

The functions $f: \mathbb{R}^{n} \rightarrow \mathbb{R}^{n}$ with $f(0)=0$, and $g: \mathbb{R}^{n} \rightarrow$ $\mathbb{R}^{n \times m}$ are considered to be sufficiently smooth and the controller $K: \mathbb{R}^{n} \rightarrow \mathbb{R}^{m}$ is a continuously differentiable function. Considering $f_{c l}(x)=f(x)+g(x) K(x), w(t)=$ $K\left(x\left(t_{k}\right)\right)-K(x(t))$ and an auxiliary output $y=\frac{\partial K}{\partial x} \dot{x}$, system (127) can be represented by

$$
\left\{\begin{aligned}
\dot{x} & =f_{c l}(x)+g(x) w \\
y & =\frac{\partial K}{\partial x}\left(f_{c l}(x)+g(x) w\right) \\
w & =\Delta_{s h} y .
\end{aligned}\right.
$$

Here we consider that $\Delta_{s h}: \mathcal{L}_{2 e}^{m}[0, \infty) \rightarrow \mathcal{L}_{2 e}^{m}[0, \infty)$, with $\Delta_{s h}$ defined similarly to (100). The main idea in $[206,207,203]$ is to re-interpret the properties of the operator $\Delta_{s h}$ in terms of "supply" functions $\mathcal{S}(y, w)$ such that

$$
\int_{t_{k}}^{t} \mathcal{S}(y(\theta), w(\theta)) d \theta \leq 0, \forall t \in\left[t_{k}, t_{k+1}\right) .
$$

The following result provides an extension of Theorem 29 to the nonlinear affine case.

Theorem 30 [206] Consider the sampled-data system (127), and the equivalent representation (128), with $\Delta_{\text {sh }}$ as given in (100). Consider the quadratic form

$$
\mathcal{S}(y, w)=\left[\begin{array}{l}
y \\
w
\end{array}\right]^{T}\left[\begin{array}{rr}
-\delta_{0}^{2} X & Y \\
Y & X
\end{array}\right]\left[\begin{array}{l}
y \\
w
\end{array}\right],
$$

with $\delta_{0}=\frac{2}{\pi} \bar{h}, 0 \prec X \in \mathbb{R}^{m \times m}$, and $0 \preceq Y \in \mathbb{R}^{m \times m}$. Consider a neighbourhood $\mathcal{D} \subset \mathbb{R}^{n}$ of the equilibrium point $x=0$, and suppose that there exist a differentiable positive definite function $V: \mathcal{D} \rightarrow \mathbb{R}^{+}$, a scalar $\alpha>0$, and class $\mathcal{K}$ functions $\beta_{1}$ and $\beta_{2}$, such that

$$
\beta_{1}(\|x\|) \leq V(x) \leq \beta_{2}(\|x\|), \quad \forall x \in \mathcal{D},
$$

and the following inequality is satisfied:

$$
\frac{\partial V}{\partial x}\left(f_{c l}(x)+g_{c l}(x) w\right)+\alpha V(x) \leq \mathcal{S}(y, w) e^{-\alpha \theta}
$$

for any $\theta \in\{0, \bar{h}\}, x \in \mathcal{D}, w \in \mathbb{R}^{m}$. Then, the equilibrium $x=0$ of system (127) is Locally Uniformly Asymptotically Stable for any sampling sequence with $t_{k+1}-t_{k} \leq \bar{h}$. 
For particular classes of systems, such as LPV, bilinear or more general polynomial sampled-data systems, the conditions of Theorem 30 can be re-written as tractable numerical criteria (LMIs or Sum-Of-Squares decomposition) $[206,207,203,202]$. These conditions can be enhanced by giving more insight into the mathematical model of the sampling operator $\Delta_{s h}$. This would lead to new characterizations of supply functions used in the dissipativity-based approach. However, finding new properties for the operator $\Delta_{s h}$ or a better way to rewrite the sampled-data system as an interconnected system has been proven to be difficult, and research is still in progress.

\subsubsection{Further reading}

Some of the elements presented in Section 4.3 concerning the use of norm-bounded approximations of the matrix exponential [79] can also be interpreted in the Input/Output approach as the application of the Small Gain Theorem to a discrete-time model. Other IQCs can be found in $[80,82]$. An approach based on IQCs for the discrete-time model has been proposed recently in [129]. For more general nonlinear networked systems, approaches considering sampling as a perturbations can be found in $[263,193]$. The boundedness properties of the sampling operator $\Delta_{s h}$ from Lemma 25 from [174] can be related with the Wirtinger's inequalities used in the time delay approach $[154,233,234]$. Motivated by the approach presented in [67] in the input delay framework, the sampling effect has been recently described by a new operator in [128].

\section{Sampling as a control parameter - an emerg- ing area}

In this section we briefly present the main research directions and some problems concerning the case when the sampling interval $h_{k}$ (or equivalently the sequence of sampling $\sigma=\left\{t_{k}\right\}_{k \in \mathbb{N}}$ ) is considered to be a control parameter that can be modified in order to ensure desired properties in terms of stability and resource utilization. From the real-time control point of view, this formulation corresponds to designing a scheduling mechanism that triggers the sampler $[12,259]$. The problem has attracted sporadically the attention of the control system's community since the early ages of sampled-data control $[125,55]$. With the spring of event- and self-triggered control techniques $[10,13,259]$ it has become a very popular topic $[103,177]$.

Let us consider the nonlinear system (2) and the controller (3) with a given sampling sequence $\sigma=\left\{t_{k}\right\}_{k \in \mathbb{N}}$. Clearly, the asymptotic stability of system holds when the sampling sequence $\sigma$ satisfies $h_{k}=t_{k+1}-t_{k} \in(0, \bar{h}]$ for all $k \in \mathbb{N}$, where $\bar{h}$ represents the MSI for which the system is asymptotically stable under arbitrary sampling. A basic problem in designing a sampling sequence $\sigma=\left\{t_{k}\right\}_{k \in \mathbb{N}}$ is to ensure the stability of the system while optimizing some Performance Index associated to the frequency of sampling. Most of the time, sampling sequences are compared in simulation based on the mean sampling interval. Given $\sigma$, one possible choice of Performance Index to be maximized could be

$$
\mathcal{J}(\sigma)=\liminf _{N \rightarrow \infty} \frac{1}{N} \sum_{k=0}^{N-1}\left(t_{k+1}-t_{k}\right) .
$$

Note that the limit inferior of the sequence is needed since, given a nonlinear system $(2),(3)$, it is not obvious that over an infinite time horizon the mean value interval converges. Generally, the goal is to find sequences that ensure stability and have the mean sampling interval larger then the maximum sampling interval admissible in the periodic and arbitrary varying case. Using the Performance Index (133), the following basic problem can be formulated:

- Problem B (Optimal sampling sequence): Consider the nonlinear system (2) and the controller (3). Design a sampling sequence $\sigma$ maximizing the Performance Index $\mathcal{J}(\sigma)$ in (133) while ensuring the stability of the closed-loop system $(1),(2),(3),(4)$.

Of course, the problem makes sense only for systems where the control action is necessary. It is not meaningful for open-loop stable systems, where the sampling interval can be made arbitrarily large. Various alternative formalizations of Problem B can be imagined by considering other performance indexes or Cost Functions (e.g. $\left.\mathcal{J}_{c}(\sigma)=\sum_{k=0}^{\infty} e^{-\left(t_{k+1}-t_{k}\right)}\right)$ to be maximized or minimized (see for instance $[117,160]$ for a finite horizon formulation). A stochastic formulation of the problem can be found in $[44,180]$. It is possible to formulate more complex problems in which one needs to find simultaneously the sampling sequence and system input, as in the minimum attention control formulation [33,54,162], or to optimise not only the sampling cost but also a more classical performance index (LQR, LQG, $\mathcal{L}_{2}$-gain, etc.) $[14,104,8,93]$.

While the research in the case of arbitrary sampling has reached an advanced phase of development, Problem B is largely open. Due to the complexity of Problem B, simplified versions are under study. For example, stability of sampled-data systems over periodic sequences of sampling has been investigated in $[125,146,232]$. The optimization of sampling sequences over a finite horizon has been considered since the early works in $[117,160]$. For both practical and theoretical reasons, the design of state-dependent (closed-loop) sampling sequences, in which the sampling is triggered according to the system state, represents a topic of interest. Basic ideas appeared in the ' $60 \mathrm{~s}$ in the context of adaptive sampling $[55,47]$ and the topic is currently under study in the framework of event-/self-triggered control [10,13,259,12,103,177]. 


\subsection{Event-Triggered (ET) Control}

The basic idea of event-triggered control schemes $[10,13,14,103,177]$ is to continuously monitor the system state and to trigger the sampling only when necessary, according to the desired performance of the system. A sampling event is generated when the system's state crosses some frontier in the state-space. Let us re-consider the hybrid model of an LTI sampled-data system

$$
\left\{\begin{array}{l}
\dot{x}=A x+B K \hat{x} \\
\dot{\hat{x}}=0 \\
\dot{\tau}=1 \\
x^{+}=x \\
\hat{x}^{+}=x \\
\tau^{+}=0
\end{array}\right\} \quad(x, \hat{x}, \tau) \in C,
$$

where $\hat{x}$ represents the sampled version of the state and $\tau$ the clock measuring the time since the last sampling instant. In the classical time-triggered sampling context (53), the sets $C$ and $D$ implicitly indicating the sampling moments are defined only according to the clock variable $\tau$ : when uniform sampling with period $T$ is considered, $C$ is defined by $\tau \in[0, T]$ and $D$ by $\tau=T$. In event-triggered control the idea is to define the sampling triggering sets according to the state variable $x$ and $\hat{x}$. For example, it may be of interest to trigger only when the error $x-\hat{x}$ becomes too large with respect to the system state, i.e. when $\left\|x(t)-x\left(t_{k}\right)\right\| \geq \gamma\|x(t)\|$ where $\gamma>0$ is a design parameter (see [250]). For this example the sets $C$ and $D$ are:

$$
\begin{aligned}
& C=\left\{(x, \hat{x}, \tau) \in \mathbb{R}^{n} \times \mathbb{R}^{n} \times \mathbb{R}:\|x-\hat{x}\| \leq \gamma\|x\|\right\}, \\
& D=\left\{(x, \hat{x}, \tau) \in \mathbb{R}^{n} \times \mathbb{R}^{n} \times \mathbb{R}:\|x-\hat{x}\| \geq \gamma\|x\|\right\} .
\end{aligned}
$$

Various other types of triggering conditions have been proposed in the literature: send-on-delta (Lesbegue sampling, absolute triggering) [14,209,37], send-onenergy [175], send-on-area [176], Lyapunov sampling $[260,238,63,221]$, etc.

Note that in event-triggering control, the sampling sequence $\sigma=\left\{t_{k}\right\}_{k \in \mathbb{N}}$ is implicitly defined as:

$$
t_{k+1}=\min \left\{t: t \geq t_{k},(x, \hat{x}, \tau) \in D\right\} .
$$

The value $h^{*}$ for which $t_{k+1}-t_{k} \geq h^{*}$ for all $k \in \mathbb{N}$ and all initial conditions is called the minimum interevent time. In the general case the implicit definition of the sampling sequence does not guarantee anything about the "well posedness" of the closed-loop system in terms of existence of solutions, or concerning the existence of a minimum interval between two consecutive events. In particular cases of event-triggered con- trol Zeno phenomena may occur, i.e. the minimum interevent time $h^{*}$ is zero ${ }^{6}[162,51,25]$. This represents an important drawback since the system is converging to a continuous-time control implementation instead of a sampled-data one. To avoid it, various systematic design methodologies for event-triggered control with stability guarantees and no Zeno behavior have been proposed: see $[250,266,264,159]$ based on the Input/Output stability approach, $[51,238,65,221]$ using hybrid models, $[271,215,63]$ based on the time-delay approach. Note that Zeno phenomena can be easily avoided by including restrictions on the clock variable when defining the jump set $D$. For example, one may add next to the constraints on $x$ and $\hat{x}$, a constraint that guarantees that sampling occurs only if $\tau$ is greater than some minimum desired inter-execution time [65,63,221]. Additionally, the triggering condition may be verified on a discrete sequence of time, as in the Periodic Event-Trigger (PET) control $[101,218,102]$, or in [57], where the event-triggered control problem is formulated directly in discrete-time.

\subsection{Self-Triggered (ST) Control}

The term self-triggered control was initially proposed by [259] in the context of real time systems. The recent articles $[264,6]$ have attracted the attention of the control system community. Note that basic ideas related to selftriggered control appeared in the '60s (see [55,117,47] and the references therein). We point also to the pioneering work in [118] where elements concerning the use of Lyapunov arguments for the design of self-triggering control laws can be found.

In self-triggering, at each sampling time it is computed both the sampled-data control value (to be sent to the actuators) and the next sampling instant. The main idea is to use the value of the state at sampling times and knowledge about the system dynamics in order to predict the next time instant a control update is needed. A self-triggering control scheme is described by a sampling function $h: \mathbb{R}^{n} \rightarrow \mathbb{R}^{+} \backslash\{0\}$ which, at each sampling time $t_{k}, k \in \mathbb{N}$, indicates the value of the current sampling interval according to the system state. The sampling sequence $\sigma=\left\{t_{k}\right\}_{k \in \mathbb{N}}$ is formulated explicitly as

$$
t_{k+1}=t_{k}+h\left(x_{k}\right),
$$

where $x_{k}=x\left(t_{k}\right)$. Very often, the synthesis of a selftriggered control scheme is based on a pre-existing eventtriggered control mechanism. In this context, it is aimed at designing the sampling function by pre-computing, at each sampling instant, an estimation of the next time a sampling event has to be generated. For the example of the LTI system (12) with the event-triggered control condition $\left\|x(t)-x\left(t_{k}\right)\right\| \geq \gamma\|x(t)\|$, one may want to

\footnotetext{
6 the system requires infinitely fast sampling
} 
design the sampling function:

$$
\begin{gathered}
h\left(x_{k}\right)=\sup \{\theta>0: \\
\forall s \leq \theta\},
\end{gathered}
$$

where $\Lambda(\theta)=e^{A \theta}+\int_{0}^{\theta} e^{A s} d s B K$. An important issue is the complexity of the algorithms used for the online implementation of the sampling function $h(x)$. Even for the simple case (137), the algorithms may be quite complex since they involve solving hyperbolic inequalities. In practice, simple approximations of such sampling function must be used.

Self-triggered control mechanisms with stability guarantees have been proposed in $[264,265,6,64,23]$ using the Input/Output stability approach, in $[166,161,255]$ using discrete-time Lyapunov functions, in $[61,63]$ using convex embeddings, in [219] using a hybrid formulation and in [62] using a time-delay system approach.

\subsection{Relations with the arbitrary sampling problem}

A basic problem in both event- and self-triggered control is to design the trigger (or the sampling map $h\left(x_{k}\right)$ ) so as to enlarge the minimum inter-event time $h^{*}$ while guaranteeing the stability of the system. Providing a quantitative estimation of the minimum inter-event time $h^{*}$ guarantees the existence of a sub-optimal solution to Problem B with a performance index $J \geq h^{*}$. Recently, connections between the arbitrary sampling problem (Problem A) and Problem B have been made in $[60,61,63,221,252,50]$. It has been shown that, for some Lyapunov-based triggering conditions, the minimum inter-event time $h^{*}$ corresponds to the Maximum Sampling Interval $\bar{h}$ admissible in the arbitrary sampling configuration. This issue is interesting since triggering control schemes could be constructed by upperbounding the derivatives of Lyapunov functions, as the ones used for solving Problem A. See, for instance, the results in $[63,62]$ where triggering mechanisms are optimized off-line using LMI criteria so as to enlarge the minimum inter-event time. However, the potential of the approaches used for the arbitrary sampling problem is far from being fully exploited. The tools presented in Section 4 may be useful for various aspects in Problem B: deriving new event-/self-triggering mechanisms, providing less conservative estimations of the minimum inter-event time $h^{*}$, etc.

\section{Conclusion}

This article has presented basic concepts and recent research directions in sampled-data systems: time-delay, hybrid, discrete-time and input-output models; Lyapunov and frequency domain methods for the stability of systems with arbitrary sampling intervals; converse
Lyapunov theorems and constructive numerical criteria. It is to be emphasized that this overview is far from being exhaustive. The research topic of systems with time-varying sampling is still wide open and continuously growing. In particular, the control of sampling is presently receiving a lot of attention, as it was shown in Section 5. It is worth noticing that the subject lies at the intersection of four important axes in Control Theory (time-delay, hybrid, LPV and input-output approaches) and we hope this will have a stimulating impact in the control community. Methods and tools are being transferred from one approach to another and the perspectives of cross-fertilisation and generalization are numerous.

\section{References}

[1] A. Ahmadi, R. Jungers, P. Parrilo, and M. Roozbehani. Joint spectral radius and path-complete graph Lyapunov functions. SIAM Journal on Control and Optimization, 52(1):687-717, 2014.

[2] A. Ahmadi and P. Parrilo. Non-monotonic Lyapunov functions for stability of discrete time nonlinear and switched systems. In 47th IEEE Conference on Decision and Control, pages 614-621, Cancun, Mexico, 2008.

[3] T. Ahmed-Ali, R. Postoyan, and F. Lamnabhi-Lagarrigue. Continuous - discrete adaptive observers for state affine systems. Automatica, 45(12):2986-2990, 2009.

[4] L.I. Allerhand and U. Shaked. Robust stability and stabilization of linear switched systems with dwell time. IEEE Transactions on Automatic Control, 56(2):381-386, 2011.

[5] V. Andrieu and M. Nadri. Observer design for Lipschitz systems with discrete-time measurements. In 49th IEEE Conference on Decision and Control, pages 6522-6527, Atlanta, Georgia, USA, 2010.

[6] A. Anta and P. Tabuada. To sample or not to sample: selftriggered control for nonlinear systems. IEEE Transactions on Automatic Control, 55(9):2030-2042, 2010.

[7] P. Antsaklis and J. Baillieul. Special issue on technology of networked control systems. Proceedings of the IEEE, 95(1):5-8, 2007.

[8] D. Antunes and W.P.M.H. Heemels. Rollout eventtriggered control: Beyond periodic control performance. IEEE Transactions on Automatic Control, 59(12):32963311, 2014.

[9] Y. Ariba and F. Gouaisbaut. An augmented model for robust stability analysis of time-varying delay systems. International Journal of Control, 82(9):1616-1626, 2009.

[10] K.E. Årzén. A simple event-based pid controller. In 14th IFAC World Congress, volume 18, pages 423-428, Beijing, China, 1999.

[11] A. Astolfi, D. Nesic, and A.R. Teel. Trends in nonlinear control. In 47th IEEE Conference on Decision and Control, pages 1870-1882, Cancun, Mexico, 2008.

[12] K.J. Åström. Event based control. In A. Astolfi and L. Marconi, editors, Analysis and Design of Nonlinear Control Systems, pages 127-147. Springer Berlin Heidelberg, 2008. 
[13] K.J. Åström and B. Bernhardsson. Comparison of periodic and event based sampling for first-order stochastic systems. In 14th IFAC World congress, volume 11, pages 301-306, Cape Town, South Africa, 1999.

[14] K.J. Åström and B. Bernhardsson. Comparison of Riemann and Lebesgue sampling for first order stochastic systems. In 41st IEEE Conference on Decision and Control, volume 2, pages 2011-2016, Las Vegas, Nevada, USA, 2002.

[15] K.J. Åström and B. Wittenmark. Adaptive control. Addison-Wesley series in electronic engineering (Control engineering), 1989.

[16] K.J. Åström and B. Wittenmark. Computer controlled systems: theory and design. Prentice Hall, 1996.

[17] D. Bainov and P. Simeonov. Impulsive differential equations: periodic solutions and applications, volume 66 . CRC Press, 1993.

[18] A. Balluchi, P. Murrieri, and A. Sangiovanni-Vincentelli. Controller synthesis on non-uniform and uncertain discretetime domains. In Hybrid Systems: Computation and Control, pages 118-133. Springer, 2005.

[19] B. Bamieh and J. Pearson. A general framework for linear periodic systems with applications to $H_{\infty}$ sampleddata control. IEEE Transactions on Automatic Control, 37(4):418-435, 1992.

[20] B. Bamieh, J. Pearson, B.A. Francis, and A. Tannenbaum. A lifting technique for linear periodic systems with applications to sampled-data control. Systems \& Control Letters, 17(2):79-88, 1991.

[21] I. Barbălat. Systèmes d'équations différentielles d'oscillations nonlinéaires. Revue Roumaine de Mathématiques Pures et Appliquées, 4:267-270, 1959.

[22] N.W. Bauer, P.J.H. Maas, and W.P.M.H. Heemels. Stability analysis of networked control systems: A sum of squares approach. Automatica, 48:1514-1524, 2012.

[23] M.D. Di Benedetto, S. Di Gennaro, and A. D'Innocenzo. Digital self-triggered robust control of nonlinear systems. International Journal of Control, 86(9):1664-1672, 2013.

[24] V. Blondel and Y. Nesterov. Computationally efficient approximations of the joint spectral radius. SIAM Journal on Matrix Analysis and Applications, 27(1):256-272, 2005.

[25] D.P. Borgers and W.P.M.H. Heemels. Event-separation properties of event-triggered control systems. IEEE Transactions on Automatic Control, 59:2644-2656, 2014.

[26] C. Briat. Convergence and equivalence results for the Jensen's inequality-application to time-delay and sampleddata systems. IEEE Transactions on Automatic Control, 56(7):1660-1665, 2011.

[27] C. Briat. Convex conditions for robust stability analysis and stabilization of linear aperiodic impulsive and sampleddata systems under dwell-time constraints. Automatica, 49(11):3449-3457, 2013.

[28] C. Briat. Theoretical and numerical comparisons of looped functionals and clock-dependent lyapunov functionsthe case of periodic and pseudo-periodic systems with impulses. International Journal of Robust and Nonlinear Control, 2015.

[29] C. Briat and U. Jönsson. Dynamic equations on timescale: application to stability analysis and stabilization of aperiodic sampled-data systems. In 18th IFAC World Congress, pages 11374-11379, Milano, Italy, 2011.

[30] C. Briat and A. Seuret. Convex dwell-time characterizations for uncertain linear impulsive systems. IEEE Transactions on Automatic Control, 57(12):3241-3246, 2012.
[31] C. Briat and A. Seuret. A looped-functional approach for robust stability analysis of linear impulsive systems. Systems $\&$ Control Letters, 61(10):980-988, 2012.

[32] C. Briat and A. Seuret. Affine minimal and mode-dependent dwell-time characterization for uncertain switched linear systems. IEEE Transactions on Automatic Control, 58(5):1304-1310, 2013.

[33] R.W. Brockett. Minimum attention control. In 36th IEEE Conference on Decision and Control, 1997, volume 3, pages 2628-2632, San Diego, California, USA, 1997.

[34] L. Burlion, T. Ahmed-Ali, and F. Lamnabhi-Lagarrigue. On the stability of a class of nonlinear hybrid systems. Nonlinear Analysis: Theory, Methods 86 Applications, 65(12):2236-2247, 2006.

[35] D. Carnevale, A.R. Teel, and D. Nešić. A Lyapunov proof of an improved maximum allowable transfer interval for networked control systems. IEEE Transactions on Automatic Control, 52(5):892-897, 2007.

[36] A. Cela, M. Ben Gaid, X.G. Li, and S.I. Niculescu. Optimization of the hyper-sampling sequence for DCESs. In Optimal Design of Distributed Control and Embedded Systems, Communications and Control Engineering, pages 207-221. Springer, 2014.

[37] A. Cervin and K.J. Aström. On limit cycles in event-based control systems. In 46th IEEE Conference on Decision and Control, pages 3190-3195, New Orleans, Louisiana, USA, 2007.

[38] J. Chen, K.H. Johansson, S. Olariu, I.C. Paschalidis, and I. Stojmenovic. Guest editorial special issue on wireless sensor and actuator networks. IEEE Transactions on Automatic Control, 56(10):2244-2246, 2011.

[39] T. Chen and B. Francis. Optimal sampled-data control systems. Springer, 1993.

[40] Z. Chen and H. Fujioka. Performance analysis of nonlinear sampled-data emulated controllers. IEEE Transactions on Automatic Control, 59(10):2778-2783, 2014.

[41] M. Cloosterman, N. van de Wouw, M. Heemels, and H. Nijmeijer. Robust stability of networked control systems with time-varying network-induced delays. In 45th IEEE Conference on Decision and Control, pages 4980-4985, San Diego, California, USA, 2006.

[42] M. Cloosterman, N. van de Wouw, W.P.M.H. Heemels, and H. Nijmeijer. Stability of networked control systems with uncertain time-varying delays. IEEE Transactions on Automatic Control, 54(7):1575-1580, 2009.

[43] M.B.G. Cloosterman, L. Hetel, N. van de Wouw, W.P.M.H. Heemels, J. Daafouz, and H. Nijmeijer. Controller synthesis for networked control systems. Automatica, 46(10):15841594, 2010.

[44] R. Cogill, S. Lall, and J. Hespanha. A constant factor approximation algorithm for event-based sampling. In American Control Conference, pages 305-311, New York City, USA, 2007.

[45] J. Daafouz and J. Bernussou. Parameter dependent Lyapunov functions for discrete time systems with time varying parametric uncertainties. Systems $\&$ control letters, 43(5):355-359, 2001.

[46] D.B. Dacic and D. Nešić. Quadratic stabilization of linear networked control systems via simultaneous protocol and controller design. Automatica, 43(7):1145-1155, 2007.

[47] M. de la Sen. Non-periodic and adaptive sampling. A tutorial review. Informatica, 7(2), 1996. 
[48] N. Van de Wouw, P. Naghshtabrizi, M. Cloosterman, and J. Hespanha. Tracking control for sampled-data systems with uncertain time-varying sampling intervals and delays. International Journal of Robust and Nonlinear Control, 20(4):1680-1685, 2010.

[49] T.N. Dinh, V. Andrieu, M. Nadri, and U. Serres. Continuous-discrete time observer design for Lipschitz systems with sampled measurements. IEEE Transactions on Automatic Control, 60(3):787-792, 2015.

[50] V.S. Dolk, D.P. Borgers, and W.P.M.H. Heemels. Outputbased and decentralized dynamic event-triggered control with guaranteed $\mathcal{L}_{p}$-gain performance and zeno-freeness. IEEE Transactions on Automatic Control, 2017, to appear.

[51] M.C.F. Donkers and W.P.M.H. Heemels. Output-based event-triggered control with guaranteed-gain and improved and decentralized event-triggering. IEEE Transactions on Automatic Control, 57(6):1362-1376, 2012.

[52] M.C.F. Donkers, W.P.M.H. Heemels, N. Van De Wouw, and L. Hetel. Stability analysis of networked control systems using a switched linear systems approach. IEEE Transactions on Automatic Control, 56(9):2101-2115, 2011.

[53] M.C.F. Donkers, L. Hetel, W.P.M.H. Heemels, N. van de Wouw, and M. Steinbuch. Stability analysis of networked control systems using a switched linear systems approach. In 12th International Conference on Hybrid Systems: Computation and Control, pages 150-164, San Francisco, California, USA, 2009.

[54] M.C.F. Donkers, P. Tabuada, and W.P.M.H. Heemels. On the minimum attention control problem for linear systems: A linear programming approach. In 50th IEEE Conference on Decision and Control and European Control Conference, pages 4717-4722, Orlando, Florida, USA, 2011.

[55] R.C. Dorf, M.C. Farren, and C. Phillips. Adaptive sampling frequency for sampled-data control systems. IRE Transactions on Automatic Control, 7(1):38-47, 1962.

[56] G. E. Dullerud and S. Lall. Asynchronous hybrid systems with jumps - analysis and synthesis methods. Systems $\mathcal{G}$ Control Letters, 37(2):61-69, 1999.

[57] A. Eqtami, D. V. Dimarogonas, and K.J. Kyriakopoulos. Event-triggered control for discrete-time systems. In American Control Conference, pages 4719-4724, Baltimore, Maryland, USA, 2010.

[58] F. Felicioni and S. Junco. A Lie algebraic approach to design of stable feedback control systems with varying sampling rate. In 17th IFAC World Congress, Seoul, Korea, 2008.

[59] F. Ferrante, F. Gouaisbaut, R. Sanfelice, and S. Tarbouriech. An observer with measurement-triggered jumps for linear systems with known input. In IFAC World Congress, Cape Town, South Africa, 2014.

[60] C. Fiter, L. Hetel, W. Perruquetti, and J.P. Richard. State dependent sampling: an LMI based mapping approach. In 18th IFAC World Congress, Milan, Italy, 2011.

[61] C. Fiter, L. Hetel, W. Perruquetti, and J.P. Richard. A state dependent sampling for linear state feedback. Automatica, 48(8):1860-1867, 2012.

[62] C. Fiter, L. Hetel, W. Perruquetti, and J.P. Richard. Statedependent sampling for perturbed time-delay systems. In 51st IEEE Conference on Decision and Control, Maui, Hawaii, USA, 2012.

[63] C. Fiter, L. Hetel, W. Perruquetti, and J.P. Richard. A robust stability framework for LTI systems with timevarying sampling. Automatica, 54(0):56 - 64, 2015.
[64] F. Forni, S. Galeani, D. Nešić, and L. Zaccarian. Lazy sensors for the scheduling of measurement samples transmission in linear closed loops over networks. In 49th IEEE Conference on Decision and Control $(C D C)$, pages 6469-6474, 2010

[65] F. Forni, S. Galeani, D. Nešić, and L. Zaccarian. Eventtriggered transmission for linear control over communication channels. Automatica, 50(2):490-498, 2014.

[66] E. Fridman. Use of models with aftereffect in the problem of the design of optimal digital-control systems. Automation and remote control, 53(10):1523-1528, 1992.

[67] E. Fridman. A refined input delay approach to sampleddata control. Automatica, 46(2):421-427, 2010.

[68] E. Fridman. Introduction to Time-Delay Systems. Springer, 2014.

69] E. Fridman and N. Bar Am. Sampled-data distributed $\mathcal{H}_{\infty}$ control of transport reaction systems. SIAM Journal on Control and Optimization, 51(2):1500-1527, 2013.

70] E. Fridman and A. Blighovsky. Sampled-data control of a class of semilinear parabolic systems. Automatica, 48:826$836,2012$.

71] E. Fridman and S.I. Niculescu. On complete LyapunovKrasovskii functional techniques for uncertain systems with fast-varying delays. International Journal of Robust and Nonlinear Control, 18(3):364-374, 2008.

[72] E. Fridman, A. Seuret, and J.P Richard. Robust sampleddata stabilization of linear systems: An input delay approach. Automatica, 40(8):1441-1446, 2004.

73] E. Fridman and U. Shaked. Sampled-data $H_{\infty}$ statefeedback control of systems with state delays. International Journal of Control, 73(12):1115-1128, 2000.

74] E. Fridman and U. Shaked. A descriptor system approach to $H_{\infty}$ control of linear time-delay systems. IEEE Transactions on Automatic Control, 47(2):253-270, 2002.

75] E. Fridman and U. Shaked. An improved stabilization method for linear time-delay systems. IEEE Transactions on Automatic Control, 47(11):1931-1937, 2002.

[76] E. Fridman and U. Shaked. Delay-dependent stability and $H_{\infty}$ control: constant and time-varying delays. International Journal of Control, 76(1):48-60, 2003.

[77] E. Fridman and U. Shaked. Input-output approach to stability and $L_{2}$-gain analysis of systems with time-varying delays. Systems \& Control Letters, 55(12):1041-1053, 2006.

[78] M. Fu, H. Li, and S.I. Niculescu. Robust stability and stabilization of time-delay systems via integral quadratic constraint approach. In Stability and Control of Timedelay Systems, volume 228 of Lecture Notes in Control and Information Sciences, pages 101-116. Springer, 1998.

[79] H. Fujioka. A discrete-time approach to stability analysis of systems with aperiodic sample-and-hold devices. IEEE Transactions on Automatic Control, 54(10):2440-2445, 2009.

[80] H. Fujioka. Some open issues in continuous-time IQC approach to a class of networked control systems. In ICCASSICE, pages 2183-2186, Fukuoka, Japan, 2009.

[81] H. Fujioka. Stability analysis of systems with aperiodic sample-and-hold devices. Automatica, 45(3):771-775, 2009.

[82] H. Fujioka. Stability verification of non-uniformly sampleddata systems with a new IQC in continuous-time domain. In 11th International Conference on Control, Automation and Systems, Gyeonggi-do, Korea, 2011. 
[83] H. Fujioka and T. Nakai. Stabilising systems with aperiodic sample-and-hold devices: state feedback case. IET control theory $\&$ applications, 4(2):265-272, 2010.

[84] H. Fujioka, T. Nakai, and L. Hetel. A switched Lyapunov function approach to stability analysis of non-uniformly sampled-data systems. In American Control Conference, pages 1803-1804, Baltimore, Maryland, USA, 2010.

[85] H. Fujioka and Y. Oishi. A switched Lyapunov function approach to stability analysis of non-uniformly sampleddata systems with robust LMI techniques. In 8th Asian Control Conference, pages 1487-1491, Kaohsiung, Taiwan, 2011.

[86] P. Gahinet and P. Apkarian. A linear matrix inequality approach to $H_{\infty}$ control. International journal of robust and nonlinear control, 4(4):421-448, 1994.

[87] H. Gao, T. Chen, and J. Lam. A new delay system approach to network-based control. Automatica, 44(1):39-52, 2008.

[88] H. Gao, W. Sun, and P. Shi. Robust sampled-data control for vehicle active suspension systems. IEEE Transactions on Control Systems Technology, 18(1):238-245, 2010.

[89] R. Gielen, S. Olaru, and M. Lazar. On polytopic approximations of systems with time-varying input delays. Lecture Notes in Control and Information Sciences (Nonlinear Model Predictive Control: Towards new Challenging Applications), 384:225-233, 2009.

[90] R. Gielen, S. Olaru, M. Lazar, W. Heemels, N. Van de Wouw, and S.I. Niculescu. On polytopic inclusions as a modeling framework for systems with time-varying delays. Automatica, 46(3):615-619, 2010.

[91] R. Goebel, R.-G. Sanfelice, and A.R. Teel. Hybrid dynamical systems. IEEE Control Systems Magazine, 29(2):28-93, 2009.

[92] R. Goebel, R.G. Sanfelice, and A.R. Teel. Hybrid Dynamical Systems: modeling, stability, and robustness. Princeton University Press, 2012.

[93] T. Gommans, D. Antunes, T. Donkers, P. Tabuada, and M. Heemels. Self-triggered linear quadratic control. Automatica, 50(4):1279-1287, 2014.

[94] F. Gouaisbaut and D. Peaucelle. Delay-dependent stability analysis of linear time delay systems. In IFAC Workshop on Time Delay Systems, L'Aquila, Italy, 2006.

[95] L. Grüne, K. Worthmann, and D. Nešić. Continuous-time controller redesign for digital implementation: A trajectory based approach. Automatica, 44(1):225 - 232, 2008.

[96] K. Gu, V. Kharitonov, and J. Chen. Stability of time-delay systems. Boston: Birkhauser, 2003.

[97] K. Gu and S.I. Niculescu. Survey on recent results in the stability and control of time-delay systems. Journal of Dynamic Systems, Measurement, and Control, 125(2):158$165,2003$.

[98] E. Gyurkovics. A note on wirtinger-type integral inequalities for time-delay systems. Automatica, 61(11):44 - 46, 2015.

[99] W.M. Haddad, V. Chellaboina, and S.G. Nersesov. Impulsive and hybrid dynamical systems: stability, dissipativity, and control. Princeton University Press, 2014.

[100] Y. He, Q.G. Wang, C. Lin, and M. Wu. Delay-rangedependent stability for systems with time-varying delay. Automatica, 43(2):371-376, 2007.

[101] W.P.M.H. Heemels, M.C.F. Donkers, and A.R. Teel. Periodic event-triggered control for linear systems. IEEE Transactions on Automatic Control, 58(4):847-861, 2013.
[102] W.P.M.H. Heemels, G.E. Dullerud, and A.R. Teel. A lifting approach to $\mathcal{L}_{2}$-gain analysis of periodic event-triggered and switching sampled-data control systems. In 54th IEEE Conference on Decision and Control (CDC), pages 41764182, 2015.

[103] W.P.M.H. Heemels, K.-H Johansson, and P. Tabuada. An introduction to event-triggered and self-triggered control. In 51st IEEE Conference on Decision and Control, pages 3270-3285, Maui, Hawaii, USA, 2012.

[104] T. Henningsson, E. Johannesson, and A. Cervin. Sporadic event-based control of first-order linear stochastic systems. Automatica, 44(11):2890-2895, 2008.

[105] G. Herrmann, S.K. Spurgeon, and C. Edwards. Discretization of sliding mode based control schemes. In 38th IEEE Conference on Decision and Control, volume 5, pages 4257-4262, Phoenix, Arizona, USA, 1999.

[106] G. Herrmann, S.K. Spurgeon, and C. Edwards. Discretization of a non-linear, exponentially stabilizing control law using an $L_{p}$-gain approach. In 39th IEEE Conference on Decision and Control, volume 4, pages 33983403, Sydney, New South Wales, Australia, 2000.

[107] J.P. Hespanha, P. Naghshtabrizi, and Y. Xu. A survey of recent results in networked control systems. IEEE Special Issue on Technology of Networked Control Systems, 95(1):138-162, 2007.

[108] L. Hetel, J. Daafouz, and C. Iung. Stabilization of arbitrary switched linear systems with unknown time-varying delays. IEEE Transactions on Automatic Control, 51(10):1668$1674,2006$.

[109] L. Hetel, J. Daafouz, and C. Iung. Analysis and control of LTI and switched systems in digital loops via an event-based modelling. International Journal of Control, 81(7):1125$1138,2008$.

[110] L. Hetel, J. Daafouz, J.P. Richard, and M. Jungers. Delaydependent sampled-data control based on delay estimates. Systems \&3 Control Letters, 60(2):146-150, 2011.

[111] L. Hetel, J. Daafouz, S. Tarbouriech, and C. Prieur. Stabilization of linear impulsive systems through a nearlyperiodic reset. Nonlinear Analysis: Hybrid Systems, 7(1):415, 2013.

[112] L. Hetel and E. Fridman. Robust sampled - data control of switched affine systems. IEEE Transactions on Automatic Control, 58(11):2922-2928, 2013.

[113] L. Hetel, E. Fridman, and T. Floquet. Variable structure control with generalized relays: A simple convex optimization approach. IEEE Transactions on Automatic Control, 60(2):497-502, 2015.

[114] L. Hetel, A. Kruszewski, W. Perruquetti, and J.P Richard. Discrete and intersample analysis of systems with aperiodic sampling. IEEE Transactions on Automatic Control, 56(7):1696-1701, 2011.

[115] L. Hou, A.N. Michel, and H. Ye. Some qualitative properties of sampled-data control systems. IEEE Transactions on Automatic Control, 42(12):1721-1725, 1997.

[116] D. Hristu-Varsakelis and W. Levine (Editors). Handbook of networked and embedded control systems. Boston: Birkhauser, 2005.

[117] T.C. Hsia. Analytic design of adaptive sampling control law in sampled-data systems. IEEE Transactions on Automatic Control, 19(1):39-42, 1974.

[118] P. Hsu and S. Sastry. The effect of discretized feedback in a closed loop system. In 26th IEEE Conference on Decision and Control, volume 26, pages 1518-1523, Los Angeles, California, USA, 1987. 
[119] B. Hu and A.N. Michel. Stability analysis of digital feedback control systems with time-varying sampling periods. Automatica, 36(6):897-905, 2000.

[120] L.S. Hu, J. Lam, Y.Y. Cao, and H.H. Shao. A linear matrix inequality (LMI) approach to robust $H_{2}$ sampled-data control for linear uncertain systems. IEEE Transactions on Systems, Man, and Cybernetics, Part B: Cybernetics, 33(1):149-155, 2003.

[121] T. Hu and F. Blanchini. Non-conservative matrix inequality conditions for stability/stabilizability of linear differential inclusions. Automatica, 46(1):190-196, 2010.

[122] Y.P. Huang and K. Zhou. Robust stability of uncertain timedelay systems. IEEE Transactions on Automatic Control, 45(11):2169-2173, 2000.

[123] A. Isidori. Nonlinear control systems, volume 1. Springer, 1995.

[124] M. Jun and M.G. Safonov. IQC robustness analysis for time-delay systems. International Journal of Robust and Nonlinear Control, 11(15):1455-1468, 2001.

[125] E.I. Jury and F.J. Mullin. The analysis of sampled-data control systems with a periodically time-varying sampling rate. IRE Transactions on Automatic Control, (1):15-21, 1959.

[126] P.T. Kabamba and S. Hara. Worst-case analysis and design of sampled-data control systems. IEEE Transactions on Automatic Control, 38(9):1337-1358, 1993.

[127] E. Kamen and P. Khargonekar. On the control of linear systems whose coefficients are functions of parameters. IEEE Transactions on Automatic Control, 29(1):25-33, 1984.

[128] C.-Y. Kao and D.-R. Wu. On robust stability of aperiodic sampled-data systems - an integral quadratic constraint approach. In American Control Conference, pages 48714876, Portland, Oregon, USA, 2014.

[129] C.Y. Kao and H. Fujioka. On stability of systems with aperiodic sampling devices. IEEE Transactions on Automatic Control, 58(8):2085-2090, 2013.

[130] C.Y. Kao and B. Lincoln. Simple stability criteria for systems with time-varying delays. Automatica, 40(8):14291434, 2004.

[131] C.Y. Kao and A. Rantzer. Stability analysis of systems with uncertain time-varying delays. Automatica, 43(6):959-970, 2007.

[132] I. Karafyllis and C. Kravaris. Global stability results for systems under sampled-data control. International Journal of Robust and Nonlinear Control, 19:1105-1128, 2009.

[133] I. Karafyllis and C. Kravaris. Robust global stabilisability by means of sampled-data control with positive sampling rate. International Journal of Control, 82(4):755-772, 2009.

[134] I. Karafyllis and M. Krstic. Global stabilization of feedforward systems under perturbations in sampling schedule. SIAM Journal on Control and Optimization, 50(3):1389-1412, 2012.

[135] I. Karafyllis and M. Krstic. Nonlinear stabilization under sampled and delayed measurements, and with inputs subject to delay and zero-order hold. IEEE Transactions on Automatic Control, 57(5):1141-1154, 2012.

[136] H. Khalil. Nonlinear systems. Prentice Hall, 2002.

[137] V. Kolmanovskii and A. Myshkis. Applied theory of functional differential equations. Springer, 1992.

[138] V. Kolmanovskii and A. Myshkis. Introduction to the theory and applications of functional differential equations. Springer, 1999.
[139] N. N. Krasovskiı̌. Stability of motion: applications of Lyapunov's second method to differential systems and equations with delay. Stanford university press, 1963.

[140] M. Krstic, I. Kanellakopoulos, and P.V. Kokotovic. Nonlinear and adaptive control design. Wiley, 1995.

[141] A. Kruszewski, R. Wang, and T.M. Guerra. Nonquadratic stabilization conditions for a class of uncertain nonlinear discrete time ts fuzzy models: A new approach. IEEE Transactions on Automatic Control, 53(2):606-611, 2008.

[142] D. Laila, D. Nešić, and A. Astolfi. Sampled-data control of nonlinear systems. In Advanced Topics in Control Systems Theory, volume 328 of Lecture Notes in Control and Information Science, pages 91-137. Springer London, 2006.

[143] V. Lakshmikantham, D. Baĭnov, and P.S. Simeonov. Theory of impulsive differential equations, volume 6 . World scientific, 1989.

[144] J.-W. Lee. On uniform stabilization of discrete-time linear parameter-varying control systems. IEEE Transactions on Automatic Control, 51(10):1714-1721, 2006.

[145] T.-H. Lee, J.-H. Park, H.-Y. Jung, O.-M. Kwon, and S.-M. Lee. Improved results on stability of time-delay systems using Wirtinger-based inequality. In 19th IFAC World Congress, pages 6826-6830, Cape Town, South Africa, 2014.

[146] X.G. Li, A. Cela, S. Niculescu, and A. Reama. Some problems in the stability of networked-control systems with periodic scheduling. International Journal of Control, 83(5):996-1008, 2010.

[147] X.G. Li, A. Cela, S.I. Niculescu, and Shi-Guang W. Optimization for networked control systems under the hyper-sampling period. In 13th European Control Conference, pages 2868-2873, Strasbourg, France, 2014.

[148] X.G. Li, H.G. Zhang, A. Cela, and S.I. Niculescu. On the stability analysis of sampled-data control systems: A combined continuous-time and discrete-time method. In 1st International Conference on Communications, Computing and Control Applications, pages 1-6, Hammamet, Tunisia, 2011.

[149] M.L. Liou. A novel method of evaluating transient response. Proceedings of the IEEE, 54(1):20-23, 1966.

[150] K. Liu and E. Fridman. Wirtinger's inequality and Lyapunov-based sampled-data stabilization. Automatica, 48(1):102-108, 2012.

[151] K. Liu, E. Fridman, and L. Hetel. Networked control systems in the presence of scheduling protocols and communication delays. SIAM Journal of Control and Optimization, 53(11): $1768-1788$.

[152] K. Liu, E. Fridman, and L. Hetel. Stability and -gain analysis of networked control systems under round-robin scheduling: A time-delay approach. Systems \& Control Letters, 61(5):666 - 675, 2012.

[153] K. Liu, E. Fridman, and K. Johansson. Networked control with stochastic scheduling. IEEE Transactions on Automatic Control, 60(11):3071 - 3078, 2015.

[154] K. Liu, V. Suplin, and E. Fridman. Stability of linear systems with general sawtooth delay. IMA Journal of Mathematical Control and Information, 27(4):419-436, 2010.

[155] C.V Loan. The sensitivity of the matrix exponential. SIAM Journal on Numerical Analysis, 14(6):971-981, 1977.

[156] W. Lombardi, S. Olaru, and S.I. Niculescu. Robust invariance for a class of time-delay systems with repeated 
eigenvalues. In 8th IFAC Workshop on Time Delay Systems, volume 8, pages 66-71, Sinaia, Romania, 2009.

[157] W. Lombardi, S. Olaru, S.I. Niculescu, and L. Hetel. A predictive control scheme for systems with variable timedelay. International Journal of Control, 85(7):915-932, 2012.

[158] J. Louisell. Delay differential systems with time-varying delay: New directions for stability theory. Kybernetika, 37(3):239-251, 2001.

[159] J. Lunze and D. Lehmann. A state-feedback approach to event-based control. Automatica, 46(1):211-215, 2010.

[160] R.K.T. Ma and R.A. Schlueter. Optimal control system design: The control and sampling problem. In 1976 IEEE Conference on Decision and Control including the 15th Symposium on Adaptive Processes, pages 228-233, Clearwater, Florida, USA, 1976.

[161] S. Maalej, C. Fiter, L. Hetel, and J.P. Richard. Statedependent sampling for linear time invariant systems: a discrete time analysis. In 20th IEEE Mediterranean Conference on Control and Automation, Barcelona, Spain, 2012.

[162] N. Marchand, S. Durand, and J.F.G. Castellanos. A general formula for event-based stabilization of nonlinear systems. IEEE Transactions on Automatic Control, 58(5):1332-1337, 2013.

[163] F. Mazenc, M. Malisoff, and T.N. Dinh. Robustness of nonlinear systems with respect to delay and sampling of the controls. Automatica, 49(6):1925-1931, 2013.

[164] F. Mazenc and D. Normand-Cyrot. Stabilization of linear input delayed dynamics under sampling. In 51st IEEE Conference on Decision and Control, pages 7523-7528, Maui, Hawaii, USA, 2012.

[165] F. Mazenc and D. Normand-Cyrot. Reduction model approach for linear systems with sampled delayed inputs. IEEE Transactions on Automatic Control, 58(5):1263-1268, 2013.

[166] M. Mazo Jr., A. Anta, and P. Tabuada. An ISS selftriggered implementation of linear controllers. Automatica, 46(8):1310-1314, 2010.

[167] A. Megretski. Integral quadratic constraints derived from the set-theoretic analysis of difference inclusions. In 35th IEEE Conference on Decision and Control, volume 3, pages 2389-2394, Kobe, Japan, 1996.

[168] A. Megretski and A. Rantzer. System analysis via integral quadratic constraints. IEEE Transactions on Automatic Control, 42(6):819-830, 1997.

[169] A.N. Michel. Recent trends in the stability analysis of hybrid dynamical systems. IEEE Transactions on Circuits and Systems I: Fundamental Theory and Applications, 46(1):120-134, 1999.

[170] A.N. Michel and B. Hu. Towards a stability theory of general hybrid dynamical systems. Automatica, 35(3):371384, 1999.

[171] R.H. Middleton and G.-C. Goodwin. Digital control and estimation: a unified approach, volume 56. Prentice Hall Englewood Cliffs, 1990.

[172] Y. Mikheev, V. Sobolev, and E. Fridman. Asymptotic analysis of digital control systems. Automation and Remote Control, 49(9):1175-1180, 1988.

[173] V.D. Milman and A.D. Myshkis. On the stability of motion in the presence of impulses. Siberian Mathematical Journal, $1(2): 233-237,1960$.
[174] L. Mirkin. Some remarks on the use of time-varying delay to model sample-and-hold circuits. IEEE Transactions on Automatic Control, 52(6):1109-1112, 2007.

[175] M. Miśkowicz. Sampling of signals in energy domain. In 10th IEEE Conference on Emerging Technologies and Factory Automation, volume 1, Catania, Italy, 2005.

[176] M. Miskowicz. Asymptotic effectiveness of the event-based sampling according to the integral criterion. Sensors, $7(1): 16-37,2007$.

[177] M. Miskowicz. Event-based control and signal processing. CRC Press, 2015.

[178] A.P. Molchanov and Y.S. Pyatnitskiy. Criteria of asymptotic stability of differential and difference inclusions encountered in control theory. Systems \& Control Letters, 13(1):59-64, 1989.

[179] C. Moler and C. Van Loan. Nineteen dubious ways to compute the exponential of a matrix, twenty-five years later. SIAM review, 45(1):3-49, 2003.

[180] A. Molin and S. Hirche. On the optimality of certainty equivalence for event-triggered control systems. IEEE Transactions on Automatic Control, 58(2):470-474, 2013.

[181] S. Monaco and D. Normand-Cyrot. On the sampling of a linear analytic control system. In 24th IEEE Conference on Decision and Control, volume 24, pages 1457-1462, Lauderdale, Florida, USA, 1985.

[182] S. Monaco and D. Normand-Cyrot. Issues on nonlinear digital control. European Journal of Control, 7(2):160-177, 2001.

[183] S. Monaco and D. Normand-Cyrot. Advanced tools for nonlinear sampled-data systems analysis and control. European Journal of Control, 13(23):221-241, 2007.

[184] M. Mousa, R. Miller, and A.N. Michel. Stability analysis of hybrid composite dynamical systems: Descriptions involving operators and difference equations. IEEE Transactions on Automatic Control, 31(7):603-615, 1986.

[185] G. Mustafa and T. Chen. Stabilisation of non-uniformly sampled systems via dynamic output-feedback control. IET Control Theory \& Applications, 7(2):228-233, 2013.

[186] M. Nadri, H. Hammouri, and R. M. Grajales. Observer design for uniformly observable systems with sampled measurements. IEEE Transactions on Automatic Control, 58(3):757-762, 2013.

[187] P. Naghshtabrizi, J.P. Hespanha, and A.-R. Teel. Exponential stability of impulsive systems with application to uncertain sampled-data systems. Systems \& Control Letters, 57(5):378-385, 2008.

[188] P. Naghstabrizi, J. Hespanha, and A. Teel. Stability of delay impulsive systems with application to networked control systems. Transactions of the Institute of Measurement and Control, 32(5):511-528, 2010.

[189] D. Nešić and L. Grüne. Lyapunov-based continuoustime nonlinear controller redesign for sampled-data implementation. Automatica, 41(7):1143-1156, 2005.

[190] D. Nešić, A.R. Teel, and P.V. Kokotović. Sufficient conditions for stabilization of sampled-data nonlinear systems via discrete-time approximations. Systems \& Control Letters, 38(4):259-270, 1999.

[191] D. Nešić and A. Teel. A framework for stabilization of nonlinear sampled-data systems based on their approximate discrete-time models. IEEE Transactions on Automatic Control, 49(7):1103-1122, 2004. 
[192] D. Nešić and A.R. Teel. Sampled-data control of nonlinear systems: An overview of recent results. In Perspectives in robust control, volume 268 of Lecture Notes in Control and Information Sciences, pages 221-239. Springer London, 2001.

[193] D. Nešić and A.R. Teel. Input-output stability properties of networked control systems. IEEE Transactions on Automatic Control, 49(10):1650-1667, 2004.

[194] D. Nešić and A.R. Teel. Input-to-state stability of networked control systems. Automatica, 40(12):2121-2128, 2004.

[195] D. Nešić, A.R. Teel, and D. Carnevale. Explicit computation of the sampling period in emulation of controllers for nonlinear sampled-data systems. IEEE Transactions on Automatic Control, 54(3):619-624, 2009.

[196] D. Nešić, A.R. Teel, and E.D. Sontag. Formulas relating KL stability estimates of discrete-time and sampled-data nonlinear systems. Systems $\&$ Control Letters, 38(1):49-60, 1999.

[197] S.I Niculescu. Delay effects on stability - a robust control approach, volume 269 of Lecture Notes in Control and Information Sciences. Springer, 2001.

[198] S.I. Niculescu and K. Gu. Advances in time-delay systems, volume 38. Springer, 2004.

[199] S.I. Niculescu, E.I. Verriest, L. Dugard, and J.M. Dion. Stability and robust stability of time-delay systems: A guided tour. In Stability and control of time-delay systems, pages 1-71. Springer, 1998.

[200] Y. Oishi and H. Fujioka. Stability and stabilization of aperiodic sampled-data control systems using robust linear matrix inequalities. Automatica, 46(8):1327-1333, 2010.

[201] S. Olaru and S. Niculescu. Predictive control for linear systems with delayed input subject to constraints. In 17th IFAC World Congress, Seoul, Korea, 2008.

[202] H. Omran, L. Hetel, M. Petreczky, J.P. Richard, and F. Lamnabhi-Lagarrigue. Stability analysis of some classes of input-affine nonlinear systems with aperiodic sampleddata control. Automatica, 70:266-274, 2016.

[203] H. Omran, L. Hetel, J. P. Richard, and F. LamnabhiLagarrigue. Stabilité des systèmes non linéaires sous échantillonnage apériodique. Journal Européen des Systèmes Automatisés, 47(4-8):467-482. 2014.

[204] H. Omran, L. Hetel, and J.P. Richard. Local stability of bilinear systems with asynchronous sampling. In 4th IFAC Conference on Analysis and Design, Eindhoven, The Netherlands, 2012.

[205] H. Omran, L. Hetel, J.P. Richard, and F. LamnabhiLagarrigue. Stability of bilinear sampled-data systems with an emulation of static state feedback. In 51st IEEE Conference on Decision and Control, pages 7541-7546, Maui, Hawaii, 2012.

[206] H. Omran, L. Hetel, J.P. Richard, and F. LamnabhiLagarrigue. On the stability of input-affine nonlinear systems with sampled-data control. In 13th European Control Conference, pages 2585-2590, Zurich, Switzerland, 2013.

[207] H. Omran, L. Hetel, J.P. Richard, and F. LamnabhiLagarrigue. Stability analysis of bilinear systems under aperiodic sampled-data control. Automatica, 50(4):12881295, 2014.

[208] L. Orihuela, P. Millán, C. Vivas, and F.R. Rubio. Delaydependent robust stability analysis for systems with interval delays. In American Control Conference, pages 4993-4998, Baltimore, Maryland, USA, 2010.
[209] P.-G. Otanez, J.-R. Moyne, and D.-M. Tilbury. Using deadbands to reduce communication in networked control systems. In American Control Conference, pages 3015-3020, Anchorage, Alaska, USA, 2002.

[210] D.H. Owens, Y. Zheng, and S.A. Billings. Fast sampling and stability of nonlinear sampled-data systems: Part 1. existence theorems. IMA Journal of Mathematical Control and Information, 7(1):1-11, 1990.

[211] M.J. Park, O.M. Kwon, J.H. Park, and S.M. Lee. A new augmented Lyapunov-Krasovskii functional approach for stability of linear systems with time-varying delays. Applied Mathematics and Computation, 217(17):7197-7209, 2011.

[212] O.M. Park, M.J.and Kwon, J.H. Park, S.M. Lee, and E.J. Cha. Stability of time-delay systems via Wirtinger-based double integral inequality. Automatica, 55:204-208, 2015.

[213] P.G. Park, J. W. Ko, and C. Jeong. Reciprocally convex approach to stability of systems with time-varying delays. Automatica, 47:235-238, 2011.

[214] P.G. Park, W.I. Lee, and S. Y. Lee. Auxiliary functionbased integral inequalities for quadratic functions and their applications to time-delay systems. Journal of the Franklin Institute, 2015.

[215] C. Peng and Q.L. Han. A novel event-triggered transmission scheme and $\mathcal{L}_{2}$ control co-design for sampled-data control systems. IEEE Transactions on Automatic Control, 58(10):2620-2626, 2013.

[216] C. Peng, Q.L. Han, D. Yue, and E. Tian. Sampled-data robust $H_{\infty}$ control for $\mathrm{T}-\mathrm{S}$ fuzzy systems with time delay and uncertainties. Fuzzy Sets and Systems, 179(1):20-33, 2011.

[217] I.R. Petersen and R. Tempo. Robust control of uncertain systems: Classical results and recent developments. Automatica, 50(5):1315-1335, 2014.

[218] R. Postoyan, A. Anta, W.P.M.H. Heemels, P. Tabuada, and D. Nesic. Periodic event-triggered control for nonlinear systems. In 52nd IEEE Conference on Decision and Control, pages 7397-7402, Florence, Italy, 2013.

[219] R. Postoyan, A. Anta, D. Nešić, and P. Tabuada. A unifying Lyapunov-based framework for the event-triggered control of nonlinear systems. In 50th IEEE Conference on Decision and Control, pages 2559-2564, Orlando, Florida, USA, 2011.

[220] R. Postoyan and D. Nešić. A framework for the observer design for networked control systems. IEEE Transactions on Automatic Control, 57(5):1309-1314, 2012.

[221] R. Postoyan, P. Tabuada, D. Nesic, and A. Anta. A framework for the event-triggered stabilization of nonlinear systems. IEEE Transactions on Automatic Control, 60(4):982-996, 2015.

[222] S. Prajna, A. Papachristodoulou, and P. Parrilo. Introducing sostools: A general purpose sum of squares programming solver. In Proc. of the 41st IEEE Conference on Decision and Control, volume 1, pages 741-746, 2002.

[223] A. Rantzer. On the Kalman-Yakubovich-Popov lemma. Systems \& Control Letters, 28(1):7-10, 1996.

[224] B.S. Razumikhin. On the stability of systems with a delay. Prikl. Mat. Mekh, 20(4):500-512, 1956.

[225] J.P Richard. Time delay systems: an overview of some recent advances and open problems. Automatica, 39(10):16671694, 2003.

[226] D. Robert, O. Sename, and D. Simon. An $H_{\infty}$ LPV design for sampling varying controllers: Experimentation with a Tinverted pendulum. IEEE Transactions on Control Systems Technology, 18(3):741-749, 2010. 
[227] W.-J. Rugh and J.-S. Shamma. Research on gain scheduling. Automatica, 36(10):1401-1425, 2000.

[228] A. Sala. Computer control under time-varying sampling period: An LMI gridding approach. Automatica, 41(12):2077-2082, 2005.

[229] S. Sastry. Nonlinear systems: analysis, stability, and control, volume 10. Springer New York, 1999.

[230] A. Seuret. Stability analysis for sampled-data systems with a time-varying period. In 48th IEEE Conference on Decision and Control, pages 8130-8135, Shanghai, China, 2009.

[231] A. Seuret. Stability analysis of networked control systems with asynchronous sampling and input delay. In American Control Conference, pages 533-538, San Francisco, California, USA, 2011.

[232] A. Seuret. A novel stability analysis of linear systems under asynchronous samplings. Automatica, 48(1):177-182, 2012.

[233] A. Seuret and F. Gouaisbaut. Wirtinger-based integral inequality: application to time-delay systems. Automatica, 49(9):2860-2866, 2013.

[234] A. Seuret and F. Gouaisbaut. Complete quadratic Lyapunov functionals using Bessel-Legendre inequality. In 13th European Control Conference, Strasbourg, France, 2014.

[235] A. Seuret and F. Gouaisbaut. Hierarchy of LMI conditions for the stability analysis of time delay systems. Systems $\&$ Control Letters, 81:1-7, 2015.

[236] A. Seuret, F. Gouaisbaut, and E. Fridman. Stability of systems with fast-varying delay using improved wirtinger's inequality. In 52nd IEEE Conference on Decision and Control, pages 946-951, Florence, Italy, 2013.

[237] A. Seuret and M. Peet. Stability analysis of sampleddata systems using sum of squares. IEEE Transactions on Automatic Control, 58(6):1620-1625, 2013.

[238] A. Seuret, C. Prieur, and N. Marchand. Stability of non-linear systems by means of event-triggered sampling algorithms. IMA Journal of Mathematical Control and Information, 2013.

[239] H. Shao. New delay-dependent stability criteria for systems with interval delay. Automatica, 45(3):744-749, 2009.

[240] H. Shao and Q.L. Han. Less conservative delay-dependent stability criteria for linear systems with interval timevarying delays. International Journal of Systems Science, 43(5):894-902, 2012.

[241] J. Skaf and S. Boyd. Analysis and synthesis of statefeedback controllers with timing jitter. IEEE Transactions on Automatic Control, 54(3):652-657, 2009.

[242] R.E. Skelton, T. Iwasaki, and K. Grigoriadis. A Unified Algebraic Approach to Linear Control Design. Taylor \& Francis Ltd, London, UK, 1998.

[243] E.D. Sontag. Mathematical control theory: deterministic finite dimensional systems, volume 6. Springer, 1998.

[244] A. Stuart and A. Humphries. Dynamical systems and numerical analysis, volume 2. Cambridge University Press, 1998.

[245] Y.S. Suh. Stability and stabilization of nonuniform sampling systems. Automatica, 44(12):3222-3226, 2008.

[246] J. Sun, G.P. Liu, J. Chen, and D. Rees. Improved delayrange-dependent stability criteria for linear systems with time-varying delays. Automatica, 46(2):466-470, 2010.

[247] W. Sun, K. M. Nagpal, and P.P. Khargonekar. $H_{\infty}$ control and filtering for sampled-data systems. IEEE Transactions on Automatic Control, 38(8):1162-1175, 1993.
[248] V. Suplin, E. Fridman, and U. Shaked. Sampled-data $H_{\infty}$ control and filtering: Nonuniform uncertain sampling. Automatica, 43(6):1072-1083, 2007.

[249] V. Suplin, E. Fridman, and U. Shaked. $H_{\infty}$ sampled-data control of systems with time-delays. International Journal of Control, 82(2):298-309, 2009.

[250] P. Tabuada. Event-triggered real-time scheduling of stabilizing control tasks. IEEE Transactions on Automatic Control, 52(9):1680-1685, 2007.

[251] G. Tadmor. $H_{\infty}$ optimal sampled-data control in continuous time systems. International Journal of Control, 56(1):99$141,1992$.

[252] P. Tallapragada and N. Chopra. Decentralized eventtriggering for control of nonlinear systems. IEEE Transactions on Automatic Control, 59(12):3312-3324, 2014.

[253] A.R. Teel. Connections between Razumikhin-type theorems and the ISS nonlinear small gain theorem. IEEE Transactions on Automatic Control, 43(7):960-964, 1998.

[254] A.R. Teel, D. Nešić, and P.V. Kokotović. A note on inputto-state stability of sampled-data nonlinear systems. In 37th IEEE Conference on Decision and Control, volume 3, pages 2473-2478, Tampa, Florida, 1998.

[255] U. Tiberi and K.H. Johansson. A simple self-triggered sampler for perturbed nonlinear systems. Nonlinear Analysis: Hybrid Systems, 10:126-140, 2013.

[256] H. T. Toivonen. Sampled-data $H_{\infty}$ optimal control of timevarying systems. Automatica, 28(4):823-826, 1992.

[257] H.T. Toivonen. Sampled-data control of continuous-time systems with an $H_{\infty}$ optimality criterion. Automatica, 28(1):45-54, 1992.

[258] N. van de Wouw, D. Nešić, and W.P.M.H. Heemels. A discrete-time framework for stability analysis of nonlinear networked control systems. Automatica, 48(6):1144-1153, 2012.

[259] M. Velasco, J. Fuertes, and P. Marti. The self triggered task model for real-time control systems. In Work-in-Progress Session of the 24th IEEE Real-Time Systems Symposium (RTSS03), volume 384, Cancun, Mexico, 2003.

[260] M. Velasco, P. Marti, and E. Bini. On Lyapunov sampling for event-driven controllers. In 48th IEEE Conference on Decision and Control, pages 6238-6243, Shanghai, China, 2009.

[261] V. Veliov. On the time-discretization of control systems. SIAM Journal on Control and Optimization, 35(5):14701486, 1997.

[262] C. VijaySekhar and W.-M. Haddad. Exponentially dissipative nonlinear dynamical systems a nonlinear extension of strict positive realness. Mathematical Problems in Engineering, 1:25-45, 2003.

[263] G.C. Walsh, O. Beldiman, and L.G. Bushnell. Asymptotic behavior of nonlinear networked control systems. IEEE Transactions on Automatic Control, 46(7):1093-1097, 2001.

[264] X. Wang and M.-D. Lemmon. Self-triggered feedback control systems with finite-gain $\mathcal{L}_{2}$ stability. IEEE Transactions on Automatic Control, 54(3):452-467, 2009.

[265] X. Wang and M.-D. Lemmon. Self-triggering under state-independent disturbances. IEEE Transactions on Automatic Control, 55(6):1494-1500, 2010.

[266] X. Wang and M.D. Lemmon. Event design in eventtriggered feedback control systems. In 47th IEEE Conference on Decision and Control, pages 2105-2110, Cancun, Mexico, 2008. 
[267] J. Willems. Dissipative dynamical systems. part I: general theory. Archive for Rational Mechanics and Analysis, 45(5):321 - 351, 1972.

[268] B. Wittenmark, J. Nilsson, and M. Torngren. Timing problems in real-time control systems. In American Control Conference, pages 2000-2004, Seattle, Washington, USA, 1995.

[269] Y. Yamamoto. A function space approach to sampled data control systems and tracking problems. IEEE Transactions on Automatic Control, 39(4):703-713, 1994.

[270] H. Ye, A.N. Michel, and L. Hou. Stability theory for hybrid dynamical systems. IEEE Transactions on Automatic Control, 43(4):461-474, 1998.

[271] D. Yue, E. Tian, and Q.L. Han. A delay system method for designing event-triggered controllers of networked control systems. IEEE Transactions on Automatic Control, 58(2):475-481, 2013.

[272] G. Zames. On the input-output stability of time-varying nonlinear feedback systems part one: Conditions derived using concepts of loop gain, conicity, and positivity. IEEE Transactions on Automatic Control, 11(2):228-238, 1966.

[273] H. Zeng, Y. He, M. Wu, and J. She. Free-matrix-based integral inequality for stability analysis of systems with time-varying delay. IEEE Transactions on Automatic Control, 2015.

[274] W. Zhang. Stability analysis of networked control systems. $\mathrm{PhD}$ thesis, Department of Electrical Engineering and Computer Science, CASE Western Reserve University, USA, 2001.

[275] W. Zhang and M.S. Branicky. Stability of networked control systems with time-varying transmission period. In 39th Annual Allerton Conference On Communication Control And Computing, volume 39, pages 1205-1214, Monticello, Illinois, USA, 2001.

[276] W. Zhang, M.S. Branicky, and S.M. Phillips. Stability of networked control systems. IEEE Control Systems Magazine, 21(1):84-99, 2001.

[277] W.A. Zhang and L. Yu. Bibo stability and stabilization of networked control systems with short time-varying delays. International Journal of Robust and Nonlinear Control, 21(3):295-308, 2011.

[278] K. Zhou, J. C. Doyle, and K. Glover. Robust and optimal control, volume 40. Prentice Hall Upper Saddle River, NJ, 1996. 